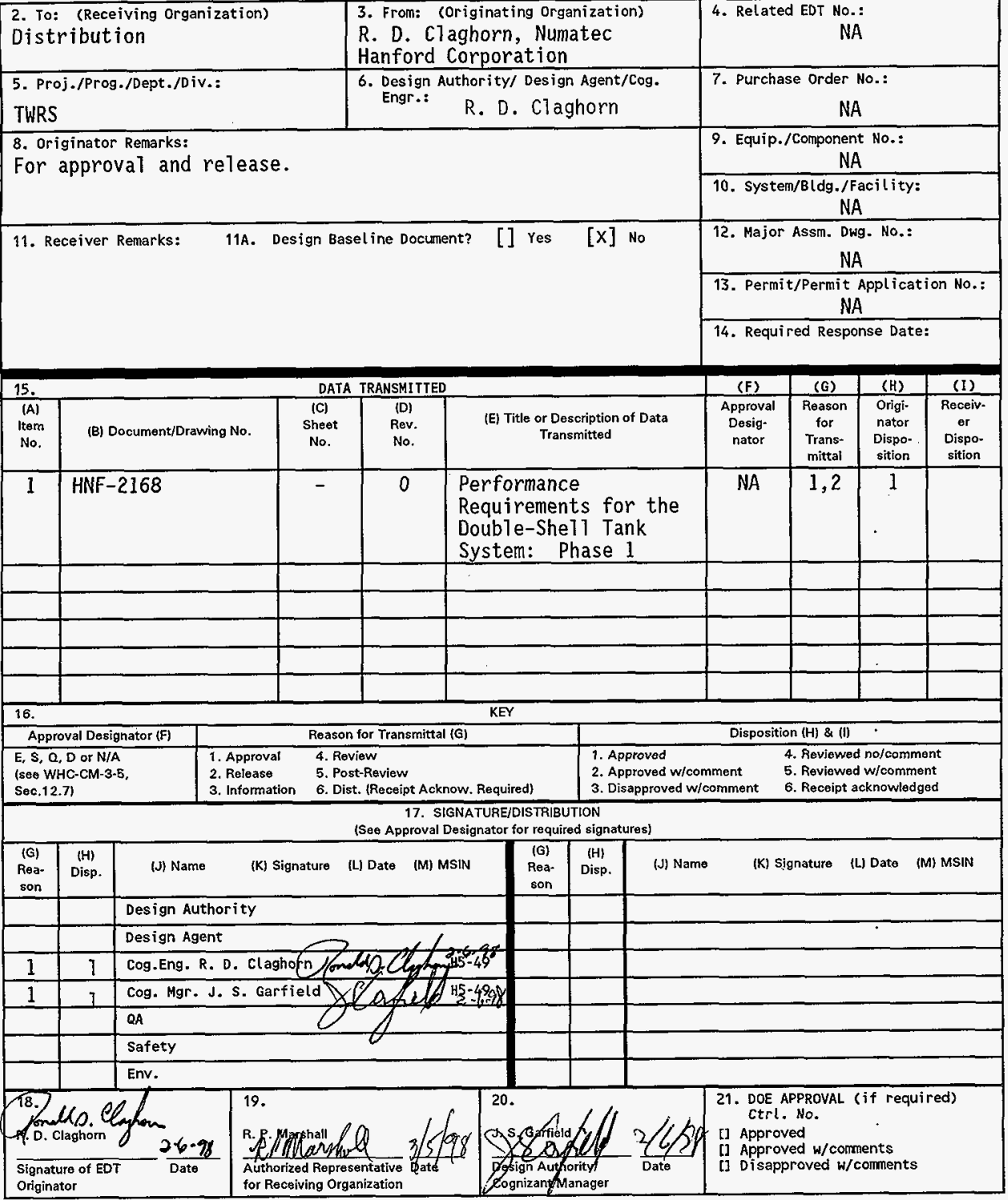




\title{
Performance Requirements for the Double-Shell Tank System: Phase 1
}

\author{
R. D. Claghorn
}

Numatec Hanford Corporation, Richland, WA 99352

U.S. Department of Energy Contract DE-AC06-96RL13200

EDT/ECN: 622702

UC: 721

Org Code: $8 C 451$

Charge Code: D2D21

B\&R Code: EW3130010

Total Pages:

81

Key Words: double-she11 tank, performance requirements

Abstract: This document establishes performance requirements for the double-she11 tank system. These requirements, in turn, will be incorporated in the System Specification for the Double-Shel7 Tank System (Grenard and Claghorn 1998).

This version of the document establishes requirements that are applicable to the first phase (Phase 1) of the Tank Waste Remediation System (TWRS) mission described in the TWRS Mission Analysis Report (Acree 1998). It does not specify requirements for either the Phase 2 mission or the double-shel 1 tank system closure period.

TRADEMARK DISCLAIMER. Reference herein to any specific commerciat product, process, or service by trade name, trademark, manufacturer, or otherwise, does not necessarily constitute or imply its endorsement, recommendation, or favoring by the United States Government or any agency thereof or its contractors or subcontractors.

Printed in the United States of America. To obtain copies of this document, contact: Document control Services, P.O. Box 950, Mailstop H6-08, Richland WA 99352, Phone (509) 372-2420; Fax (509) 376-4989.
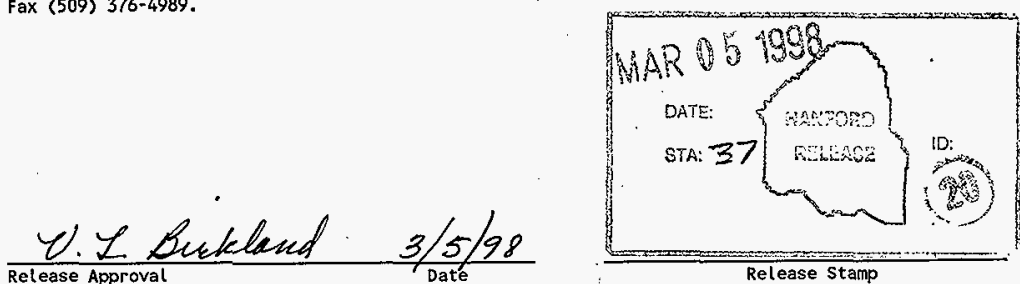

Release Stamp 
HNF-2168

Revision 0

\title{
PERFORMANCE REQUIREMENTS FOR THE DOUBLE-SHELL TANK SYSTEM: PHASE 1
}

\author{
January 1998
}

R. D. Claghorn

Numatec Hanford Corporation

Richland, Washington

Prepared for

U.S. Department of Energy

Richland, Washington 
HNF-2168

Revision 0

This page intentionally left blank. 
HNF-2168

Revision 0

CONTENTS

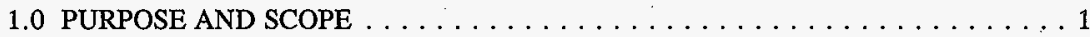

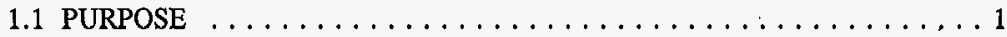

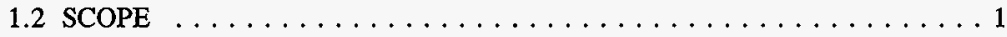

2.0 APPROACH $\ldots \ldots \ldots \ldots \ldots \ldots \ldots \ldots \ldots \ldots \ldots \ldots \ldots \ldots \ldots \ldots \ldots \ldots \ldots \ldots \ldots$

3.0 PERFORMANCE REQUIREMENTS $\ldots \ldots \ldots \ldots \ldots \ldots \ldots \ldots$

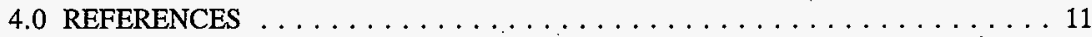

APPENDIX A - CALCULATION SHEETS $\ldots \ldots \ldots \ldots \ldots \ldots \ldots$ A-1 
HNF-2168

Revision 0

\section{LIST OF TERMS}

DOE

DST

HLW

HSTBD

HTWOS

LAW

LERF

SST

TBD

TBP

TWRS

TWRSO\&UP
U.S. Department of Energy

Double-shell tank

High-level waste

Hanford Site Technical Database

Hanford Tank Waste Operations Simulator

Low-activity waste

Liquid Effluent Retention Facility

Single-shell tank

To be determined

To be published

Tank Waste Remediation System

Tank Waste Remediation System Operation and Utilization Plan 
HNF-2168

Revision 0

\section{PERFORMANCE REQUIREMENTS FOR THE DOUBLE-SHELL TANK SYSTEM: PHASE 1}

\subsection{PURPOSE AND SCOPE}

\subsection{PURPOSE}

This document establishes performance requirements for the double-shell tank (DST) system. These requirements, in turn, will be incorporated in the System Specification for the Double-Shell Tank System (Grenard and Claghorn 1998).

\subsection{SCOPE}

This version of the document establishes requirements which are applicable to the first phase (Phase 1) of the TWRS mission described in the TWRS Mission Analysis Report (Acree 1998). It does not specify requirements for either the Phase 2 mission or the DST system closure period.

\subsection{APPROACH}

Most of the performance requirements listed in this document are derived by an analysis of Appendix $\mathrm{H}$ in the TWRS Operations and Utilization Plan (TWRSO\&UP, Kirkbride et al. 1997) as updated by the Tank Waste Remediation System Retrieval and Disposal Mission Initial Updated Baseline Summary (Swita 1998). Appendix $\mathrm{H}$ of the TWRSO\&UP lists the transfers required to meet all of the DST system objectives during Phase 1. The list of transfers includes some historical information which was also used to quantify requirements. Additional detail explaining the derivation of each requirement is given in Section 3.0.

The operations described in the TWRSO\&UP are constrained by requirements imposed by external agencies such as the U.S. Department of Energy (DOE) and the State of Washington. These constraints are listed in the System Specification for the Double-Shell Tank System (Grenard and Claghorn.1998). The configuration and the usage of the DSTs are based on the studies and decisions listed in Table 1. 
Table 1. Basis for Double-Shell Tank Usage.

\begin{tabular}{|l|l|}
\hline \multicolumn{1}{|c|}{ Title } & \multicolumn{1}{|c|}{ Content } \\
\hline $\begin{array}{l}\text { Preliminary Low-Level Waste Feed Staging } \\
\text { Plan (Certa et a1. 1996) }\end{array}$ & $\begin{array}{l}\text { Evaluates options for the staging of LAW } \\
\text { feed }\end{array}$ \\
\hline $\begin{array}{l}\text { Decision Document, Low-Level Waste Feed } \\
\text { Staging Strategy (Daling 1996) }\end{array}$ & $\begin{array}{l}\text { Documents the decision to use two double- } \\
\text { shell tanks for intermediate waste feed } \\
\text { staging and to turn over two tanks to the } \\
\text { private contractors as feed tanks. }\end{array}$ \\
\hline $\begin{array}{l}\text { Alternatives Generation and Analysis for the } \\
\text { Phase 1 Intermediate Waste Feed Staging } \\
\text { System Design Requirements (Claghorn et al. } \\
\text { 1996) }\end{array}$ & $\begin{array}{l}\text { Evaluates options. for the preparation and } \\
\text { qualification of waste within the intermediate } \\
\text { waste feed staging tanks }\end{array}$ \\
\hline $\begin{array}{l}\text { Decision Document, Phase 1 Intermediate } \\
\text { Waste Feed Staging System Design } \\
\text { Requirements (Galbraith and Daling 1997) }\end{array}$ & $\begin{array}{l}\text { Documents the selection of a mixer concept } \\
\text { for the intermediate waste feed staging tanks } \\
\text { 241-AP-102 and 241-AP-104 }\end{array}$ \\
\hline $\begin{array}{l}\text { Alternatives Generation and Analysis for } \\
\text { Waste Preparation Equipment - Phase 1 } \\
\text { Source Tanks (Claghorn et al. 1998) }\end{array}$ & $\begin{array}{l}\text { Evaluates options for the preparation of } \\
\text { waste from the source tanks for Phase 1 } \\
\text { waste feed delivery }\end{array}$ \\
\hline $\begin{array}{l}\text { Design Basis for Retrieval Equipment in } \\
\text { Tanks AN-104 and AN-105 (Boston 1997) }\end{array}$ & $\begin{array}{l}\text { Documents the selection of a mixer concept } \\
\text { for 241-AN-104 and -105. This letter also } \\
\text { outlined an operational concept for the } \\
\text { retrieval of waste from these tanks. }\end{array}$ \\
\hline $\begin{array}{l}\text { Decision Document, Waste Preparation } \\
\text { Equipment for Phase 1 Source Tanks (TBP) }\end{array}$ & TBP \\
\hline $\begin{array}{l}\text { Decision Document for Phase 1 Privatization } \\
\text { Transfer System Needs (Galbraith et al. } \\
\text { 1996) }\end{array}$ & $\begin{array}{l}\text { Evaluates options and documents the } \\
\text { selection of piping upgrades within tank } \\
\text { farms }\end{array}$ \\
\hline
\end{tabular}

LAW $=$ Low-activity waste

$\mathrm{TBP}=$ To be published . 


\subsection{PERFORMANCE REQUIREMENTS}

Table 2 lists the performance requirements, the applicable paragraph of the System Specification for the Double-Shell Tank System (Grenard and Claghorn 1998), and additional detail on how the numbers were derived. The required transfer durations in this table are, in general, based on a reasonable minimum duration as estimated in the Tank Waste Remediation System Retrieval and Disposal Mission Initial Updates Baseline Summary (Swita 1998). The minimum duration is chosen as the requirement because the program strategy is to use the balance of the available time as contingency to correct unforseen problems such as equipment failures. This strategy is intended to enhance the Waste Feed Delivery Program's chances of success.

Many of the requirements listed in Table 2 are subject to change in the event that the scenario for the conduct of Phase 1 is changed significantly. 
Table 2. Double-Shell Tank System Performance Requirements. (7 Sheets)

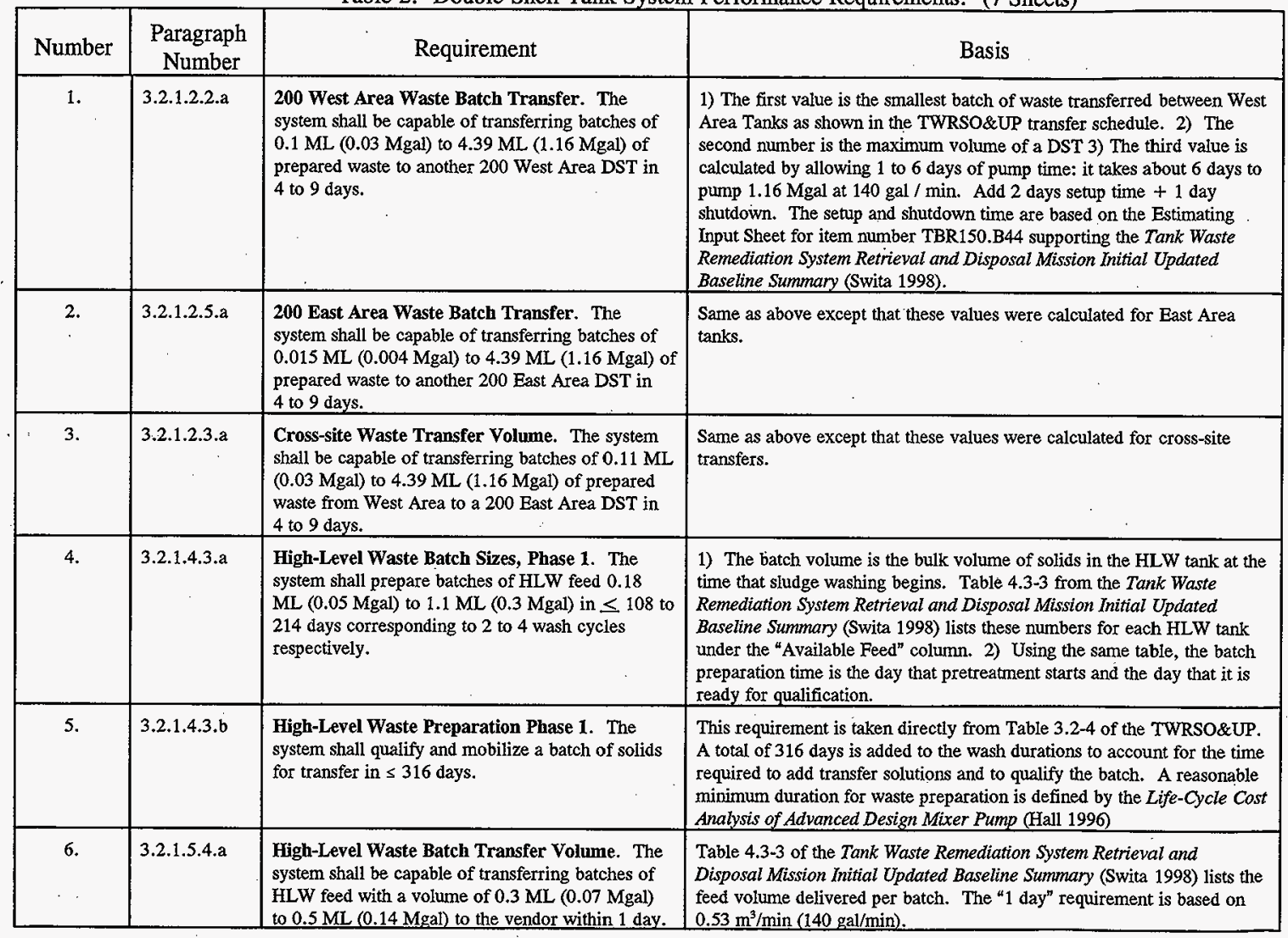


Table 2. Double-Shell Tank System Performance Requirements. (7 Sheets)

\begin{tabular}{|c|c|c|c|}
\hline Number & $\begin{array}{c}\text { Paragraph } \\
\text { Number }\end{array}$ & Requirement & Basis \\
\hline 7. & 3.2.1.2.1.c & $\begin{array}{l}\text { Insoluble Waste Preparation - West Area Tanks. } \\
\text { The system shall have the capability to mobilize } \\
\text { insoluble waste and transform it to satisfy the } \\
\text { transfer requirements of HNF-SD-WM-DQO-001, } \\
\text { Rev. } 2 \text { (Mulkey 1997) within } 14 \text { days. }\end{array}$ & $\begin{array}{l}\text { The only DST in West Area with a significant quantity of insoluble waste } \\
\text { is SY-102. A reasonable minimum duration for waste preparation is } \\
\text { defined by the rationale for assumption 'C' in Section } 4.1 \text { of the Life- } \\
\text { Cycle Cost Analysis of Advanced Design Mixer Pump (Hall 1996). }\end{array}$ \\
\hline 8. & $3.2 .1 .3 .4 . c$ & $\begin{array}{l}\text { Insoluble Waste Preparation - East Area Tanks. } \\
\text { The system shall have the capability to mobilize } \\
\text { insoluble waste and transform it to satisfy the } \\
\text { transfer requirements of HNF-SD-WM-DQO-001, } \\
\text { Rev. } 2 \text { (Mulkey 1997) in } 1 \text { day. }\end{array}$ & $\begin{array}{l}\text { Table 3.2-4 of the TWRSO\&UP shows that a minimum of } 1 \text { day is } \\
\text { allocated to the transfer which includes the waste preparation time. }\end{array}$ \\
\hline 9. & 3.2.1.2.7.a & $\begin{array}{l}\text { Low-Activity Waste Staging Batch Volumes. The } \\
\text { system shall be capable of transferring batches of } \\
1.1 \mathrm{ML}(0.3 \mathrm{Mgal}) \text { to } 3.8 \mathrm{ML} \text { ( } 1 \mathrm{Mgal}) \text { of } \\
\text { prepared waste to the LAW staging tank within } 4 \text { to } \\
9 \text { days. }\end{array}$ & $\begin{array}{l}\text { These values were calculated with the same procedure used to calculate } \\
\text { the values for Item \#1 above except that these values are based on } \\
\text { projected transfers to the LAW staging tanks: AP-102 and AP-104. }\end{array}$ \\
\hline 11. & 3.2.1.3.1.a & $\begin{array}{l}\text { Low-Activity Waste Staging Batch Size, Phase } 1 . \\
\text { The system shall be capable of blending and } \\
\text { qualifying batches of LAW feed with a volume of } \\
1.04 \mathrm{ML}(0.3 \mathrm{Mgal}) \text { to } 4.4 \mathrm{ML}(1.16 \mathrm{Mgal}) \text {. The } \\
\text { system shall blend and qualify each batch of LAW } \\
\text { feed in } \leq 113 \text { days. }\end{array}$ & $\begin{array}{l}\text { The minimum volume is based on information in Table 4.3-1 of the Tank } \\
\text { Waste Remediation System Retrieval and Disposal Mission Initial Updated } \\
\text { Baseline Summary (Swita 1998). The maximum volume is equal to the } \\
\text { tank capacity. The duration is based on the Estimating Input Sheets that } \\
\text { support the schedule given in the Tank Waste Remediation System } \\
\text { Retrieval and Disposal Mission Initial Updated Baseline Summary (Swita } \\
\text { 1998). }\end{array}$ \\
\hline
\end{tabular}


Table 2. Double-Shell Tank System Performance Requirements. (7 Sheets)

\begin{tabular}{|c|c|c|c|}
\hline Number & $\begin{array}{c}\text { Paragraph } \\
\text { Number }\end{array}$ & Requirement & Basis \\
\hline 12. & 3.2.1.3.2.a & $\begin{array}{l}\text { Low-Activity Waste Batches, Phase } 1 \text {. The } \\
\text { system shall be capable of delivering batches of } \\
\text { envelope A, B, and C LAW feed with volume of } \\
0.74 \mathrm{ML}(0.2 \mathrm{Mgal}) \text { to } 3.82 \mathrm{ML}(1 \mathrm{Mgal}) \text { to each } \\
\text { plant feed tank within } 9 \text { days. }\end{array}$ & $\begin{array}{l}\text { Same as item } 11 \text { except that this is for waste transferred to } 6 \mathrm{AP} \text { and } 8 \mathrm{AP} \\
\text { during Phase 1. 1) The minimum value corresponds to Batch } \# 7 \text { and } 2 \text { ) } \\
\text { the maximum volume corresponds to Batch \#11. 3) The } 9 \text { days is based } \\
\text { on the reasoning given for requirement \#1. }\end{array}$ \\
\hline 13. & 3.2.1.4.2.a & $\begin{array}{l}\text { Sludge Wash Supernatant Total Volume. The } \\
\text { system shall be capable of transferring a maximum } \\
\text { of } 8.7 \mathrm{ML}(2.3 \mathrm{Mgal}) \text { of wash supernate in five } \\
\text { years. }\end{array}$ & $\begin{array}{l}\text { Table } 3.2-5 \text { of the TWRSO\&UP lists the volumes of wash supernatant } \\
\text { transferred out of } 1 / 2 \mathrm{AZ} \text { and } 2 \mathrm{AY} \text { over } 5 \text { years (starting at the } \\
\text { pretreatment start date). There aren't any transfers of supernatant after } \\
6 / 2007 \text { which is } 5 \text { years after plant startup. }\end{array}$ \\
\hline 14. & $3.2 .1 .4 .2 . b$ & $\begin{array}{l}\text { Sludge Wash Supernatant Batch Volume. The } \\
\text { system shail be capable of transferring } 0.25 \mathrm{ML} \\
\text { (0.07 Mgal) to } 1.4 \mathrm{ML}(0.37 \mathrm{Mgal}) \text { of wash } \\
\text { supernate containing negligible insoluble solids in } \\
\leq 4 \text { days. }\end{array}$ & $\begin{array}{l}\text { 1) The first number is the minimum volume of wash supernatant } \\
\text { transferred out of } 1 / 2 \mathrm{AZ} \text { and } 2 \mathrm{AY} \text { according to Table } 3.2-5 \text { of the } \\
\text { TWRSO\&UP. 2) This second number is based on the maximum volume } \\
\text { from the same table. 3) The TWRSO\&UP assumes that the quantity of } \\
\text { solids entrained in the decant is insignificant. }\end{array}$ \\
\hline$\div 15$ & $3.2 .1 .2 .1 . \mathrm{b}$ & $\begin{array}{l}\text { Soluble Waste Preparation - West Area. The } \\
\text { system shall have the capability to transform } \\
\text { sufficient soluble waste to achieve a supernatant } \\
\text { sodium molarity of } 7.0 \text { in } 82 \text { days or to transform } \\
\text { all of the soluble sodium into a solution that } \\
\text { satisfies the transfer requirements of } \\
\text { HNF-SD-WM-DQO-001, Rev. } 2 \text { (Mulkey 1997). }\end{array}$ & $\begin{array}{l}\text { 1) Table 3.1-8 of the TWRSO\&UP lists the molarity of the retrieved } \\
\text { waste as it would exist in the pipeline on its way to the intermediate waste } \\
\text { feed staging tanks. 2) The time required to prepare waste is taken from } \\
\text { supporting information for the schedule in the Tank Waste Remediation } \\
\text { System Retrieval and Disposal Mission Initial Updated Baseline Summary } \\
\text { (Swita 1998). This duration does not include the time required to degas } \\
\text { or transfer. }\end{array}$ \\
\hline 16. & $3.2 .1 .3 .4 . \mathrm{b}$ & $\begin{array}{l}\text { Soluble Waste Preparation - East Area. The } \\
\text { system shall have the capability to transform } \\
\text { sufficient soluble waste the achieve a supernatant } \\
\text { sodium molarity of } 6.5 \text { to } 9.0 \text { in } 117 \text { days or to } \\
\text { transform all the soluble waste into a solution that } \\
\text { satisfies the transfer requirements of } \\
\text { HNF-SD-WM-DQO-001, Rev. } 2 \text { (Mulkey 1997). }\end{array}$ & $\begin{array}{l}\text { These values were derived using the same approach as item } 15 \text { except } \\
\text { that this is for East Area LAW source tanks. }\end{array}$ \\
\hline 17. & $3.2 .1 .2 . b$ & $\begin{array}{l}\text { Space Management Annual Waste Volume, } \\
\text { Phase 1. The system shall be capable of moving } \\
87 \text { ML ( } 23 \text { Mgal) per year of waste through the } \\
\text { end of Phase } 1 \text { for space management. }\end{array}$ & $\begin{array}{l}\text { This is the sum the maximum yearly total transferred from } \\
\text { 1) an East Area DST to another East Area DST (10 Mgal) } \\
\text { 2) AW-102 to the evaporator ( } 4 \text { Mgal) } \\
\text { 3) a West Area DST to West Area DST ( } 5 \mathrm{Mgal}) \\
\text { 4) a West Area DST to an East Area DST ( } 4 \mathrm{Mgal}) \\
\text { Note: The DST system does not move waste from the evaporator to the } \\
\text { system. }\end{array}$ \\
\hline
\end{tabular}


Table 2. Double-Shell Tank System Performance Requirements. (7 Sheets)

\begin{tabular}{|c|c|c|c|}
\hline Number & $\begin{array}{c}\text { Paragraph } \\
\text { Number }\end{array}$ & Requirement & Basis \\
\hline 18. & 3.2.1.2.1.a & $\begin{array}{l}\text { Supernatant Preparation - West Area. The } \\
\text { system shall have the capability to adjust up to } \\
4.4 \mathrm{ML}(1.16 \mathrm{Mgal}) \text { of supernatant to meet the in- } \\
\text { line transfer requirements of } \\
\text { HNF-SD-WM-DQO-001, Rev. } 2 \text { (Mulkey 1997) in } \\
\text { line. }\end{array}$ & $\begin{array}{l}\text { The volume stated is the maximum tank volume. The TWRSO\&UP } \\
\text { currently doesn't project this type of adjustment within a tank in West } \\
\text { Area. }\end{array}$ \\
\hline 20. & 3.2.3.10.a & $\begin{array}{l}\text { Water - Phase 1. The system shall obtain } 49 \mathrm{ML} \\
\text { (13 Mgal) of water from the Hanford Landlord } \\
\text { System for distribution throughout the DST system. }\end{array}$ & $\begin{array}{l}\text { This is the sum of all the water inputs over the duration of Phase } 1 \text { as } \\
\text { listed in Appendix } H \text { of the TWRSO\&UP. }\end{array}$ \\
\hline 22. & 3.2.1.3.d & $\begin{array}{l}\text { Low-Activity Waste Feed Frequency, Phase } 1 \text {. } \\
\text { The system shall be capable of mobilizing, staging, } \\
\text { blending, qualifying and delivering a batch of } \\
\text { LAW feed to the vendor in } \leq 182 \text { days from } \\
\text { sources where soluble saits must be dissolved and } \\
\leq 125 \text { days from sources consisting of } \\
\text { supernatant. }\end{array}$ & $\begin{array}{l}\text { This is the minimum duration of a feed delivery cycle as defined in the } \\
\text { Input Estimating Sheets used to support the schedule in the Tank Waste } \\
\text { Remediation System Retrieval and Disposal Mission Initial Updated } \\
\text { Baseline Summary (Swita 1998). Batches } 7 \text { and } 8 \text { are qualified in Tank } \\
\text { 241-AN-107 and therefore, not counted for this analysis. The time } \\
\text { required to degas is not included in this requirement. }\end{array}$ \\
\hline 23. & $3.2 .1 .1 .8 . \mathrm{a}$ & $\begin{array}{l}\text { Treatment Plant Waste Product Volume, } \\
\text { Phase 1. The system shall be capable of receiving } \\
0.5 \mathrm{ML} / \mathrm{yr}(0.13 \mathrm{Mgal} / \mathrm{yr}) \text { of entrained solids and } \\
{ }^{0} \mathrm{Sr} / \text { transuranic (TRU) through the end of } \\
\text { Phase 1. }\end{array}$ & $\begin{array}{l}\text { This is the maximum projected yearly total of slurry (liquids and solids) } \\
\text { returned from the private contractors. }\end{array}$ \\
\hline
\end{tabular}


Table 2. Double-Shell Tank System Performance Requirements. (7 Sheets)

\begin{tabular}{|c|c|c|c|}
\hline Number & $\begin{array}{c}\text { Paragraph } \\
\text { Number }\end{array}$ & Requirement & Basis \\
\hline 25. & 3.2.1.1.3.b & $\begin{array}{l}\text { 204-AR Batch Transfer. The system shall be } \\
\text { capable of unloading batches of } 18,900 \mathrm{~L} \\
(5,000 \mathrm{gal}) \text { in } \leq 1 \text { day. }\end{array}$ & $\begin{array}{l}\text { This is the capacity of the railcar and the typical duration required to } \\
\text { unload the car. }\end{array}$ \\
\hline 26. & 3.2.1.1.6.a & $\begin{array}{l}\text { Annual Volume of Concentrated Waste. The } \\
\text { system shall have the capacity to receive up to } \\
9.1 \mathrm{ML} \text { ( } 2.4 \mathrm{Mgal}) \text { of waste per year through } \\
\text { Phase 1. }\end{array}$ & $\begin{array}{l}\text { This is the maximum quantity of waste received from the evaporator into } \\
241-A W-106 \text { on a yearly basis as listed in Appendix } H \text { of the } \\
\text { TWRSO\&UP. }\end{array}$ \\
\hline 27. & 3.2.3.4.a & $\begin{array}{l}\text { B Plant/Waste Encapsulation and Storage } \\
\text { Facility Waste Volume. The system shall accept } \\
\text { up to } 0.2 \mathrm{ML}(0.054 \mathrm{Mgal}) \text { of liquid waste per } \\
\text { year from B Plant / WESF. }\end{array}$ & $\begin{array}{l}\text { This is the sum the maximum quantity of waste received each year from } \\
\text { B Plant and from WESF as listed in Appendix H of the TWRSO\&UP. }\end{array}$ \\
\hline 28. & 3.2.3.6.a & $\begin{array}{l}\text { Concentrated Waste Received from the } \\
\text { Evaporator. The system shall be capable of } \\
\text { accepting 3.4 ML (0.9 Mgal) of waste in } 8 \text { days. }\end{array}$ & $\begin{array}{l}\text { This is the maximum batch volume identified in Appendix } \mathrm{H} \text { of the } \\
\text { TWRSO\&UP for transfers from the Evaporator to tank } 241-\mathrm{AW}-106 \text {. }\end{array}$ \\
\hline 29. & 3.2.1.1.5.a & $\begin{array}{l}\text { East Area Liquid Waste Annual Capacity. The } \\
\text { system shall be capable of receiving up } 15 \mathrm{ML} \\
\text { (3.9 Mgal) of liquid waste per year through } \\
\text { Phase } 1 .\end{array}$ & $\begin{array}{l}\text { This value is the sum of the maximum yearly quantity of waste received } \\
\text { from each of the following facilities as listed in Appendix H of the } \\
\text { TWRSO\&UP: } \\
\text { 1) } 100 \text { Area basins } \\
\text { 2) Plutonium-Uranium Extraction Plant } \\
\text { 3) WESF and B Plant } \\
\text { 4) } 300 \text { Area } \\
\text { 5) Salt well pumping } \\
\text { 6) SST Retrieval }\end{array}$ \\
\hline 30. & $3.2 .3 .5 . \mathrm{b}$ & $\begin{array}{l}\text { East Single-Shell Tank Salt Well Liquid Waste } \\
\text { Volume. The system shall accept } 0.8 \mathrm{ML} \\
(0.2 \mathrm{Mgal}) \text { of dilute, non-complexed (DN) and } \\
\text { 3.4 ML (0.9 Mgal) of dilute, complexed (DC) } \\
\text { liquid from salt well pumping from the SSTs over } 4 \\
\text { years. }\end{array}$ & $\begin{array}{l}\text { These values are derived from the transfer schedule listed in Appendix H } \\
\text { of the TWRSO\&UP. }\end{array}$ \\
\hline 31. & 3.2.1.2.6.a & $\begin{array}{l}\text { Evaporator Feed Annual Volume. The system } \\
\text { shall be capable of providing up to } 17 \mathrm{ML} / \mathrm{yr} \\
\text { (4.4 Mgal/yr) of dilute supernate from Tank } \\
\text { 241-AW-102 for concentration. }\end{array}$ & $\begin{array}{l}\text { These values are derived from the transfer schedule listed in Appendix } H \\
\text { of the TWRSO\&UP. In this case, the value is the maximum quantity } \\
\text { provided in one year. }\end{array}$ \\
\hline
\end{tabular}


Table 2. Double-Shell Tank System Performance Requirements. (7 Sheets)

\begin{tabular}{|c|c|c|c|}
\hline Number & $\begin{array}{c}\text { Paragraph } \\
\text { Number }\end{array}$ & Requirement & Basis \\
\hline 32. & $3.2 .1 .2 .6 . b$ & $\begin{array}{l}\text { Evaporator Feed Batch Size. The maximum } \\
\text { evaporator batch size to be transferred to the } \\
\text { evaporator by the system (i.e., campaign) is } \\
5.7 \mathrm{ML}(1.5 \mathrm{Mgal}) \text {. }\end{array}$ & $\begin{array}{l}\text { These values are derived from the transfer schedule listed in Appendix } H \\
\text { of the TWRSO\&UP. }\end{array}$ \\
\hline 33. & 3.2.1.2.c & $\begin{array}{l}\text { Evaporator Feed Volume, Phase } 1 . \text { The system } \\
\text { shall be capable of delivering up to } 17 \mathrm{ML} \\
(4.4 \mathrm{Mgal}) \text { of waste per year for transfer to the } \\
\text { evaporator through the end of Phase } 1 \text {. }\end{array}$ & These are the same values as item $\# 31$. \\
\hline 34. & 3.2.1.1.4.b & $\begin{array}{l}\text { Waste Batch Size - West Area. The system shall } \\
\text { be capable of receiving batches of } 0.004 \mathrm{ML} \\
(0.001 \mathrm{Mgal}) \text { to } 2.7 \mathrm{ML}(0.72 \mathrm{Mgal}) \text { in } \leq 9 \text { days. }\end{array}$ & $\begin{array}{l}\text { This volumetric information is based on the minimum and the maximum } \\
\text { batch sizes listed in Appendix } \mathbf{H} \text { of the TWRSO\&UP for transfers from } \\
\text { the following facilities: } \\
\text { 1) 222-S Laboratory } \\
\text { 2) SST Retrieval } \\
\text { 3) T-Plant } \\
\text { 4) Plutonium Finishing Plant } \\
\text { 5) Salt well pumping } \\
\text { The duration is based on the maximum number of days required to pump } \\
\text { the waste plus } 2 \text { days setup time and one day for shutdown operations. } \\
\text { The setup and shutdown time are based on the Estimating Input Sheets } \\
\text { supporting the Tank Waste Remediation System Retrieval and Disposal } \\
\text { Mission Initial Updated Baseline Summary (Swita 1998). }\end{array}$ \\
\hline 35. & 3.2.1.1.5.b & $\begin{array}{l}\text { Liquid Waste Batch Size - East Area. The } \\
\text { system shall be capable of receiving batches of } \\
0.02 \mathrm{ML}(0.005 \mathrm{Mgal}) \text { to } 3 \mathrm{ML}(0.8 \mathrm{Mgal}) \text { in } \\
\leq 9 \text { days. }\end{array}$ & $\begin{array}{l}\text { This volumetric information is based on the minimum and the maximum } \\
\text { batch sizes listed in Appendix } H \text { of the TWRSO\&UP for transfers from } \\
\text { the following facilities: } \\
\text { 1) } 100 \text { Area basins } \\
\text { 2) Plutonium-Uranium Extraction Plant } \\
\text { 3) WESF and B Plant } \\
\text { 4) } 300 \text { Area } \\
\text { 5) Salt well pumping } \\
\text { The duration is based on the maximum number of days required to pump } \\
\text { the waste plus } 2 \text { days setup time and one day for shutdown operations. } \\
\text { The setup and shutdown time are based on the Estimating Input Sheets } \\
\text { supporting the Tank Waste Remediation System Retrieval and Disposal } \\
\text { Mission Initial Updated Baseline Summary (Swita 1998). }\end{array}$ \\
\hline 36. & 3.2.3.2.a & $\begin{array}{l}\text { Plutonium Finishing Plant Waste Annual } \\
\text { Volume. The system shall accept up to } 30,000 \mathrm{~L} \\
(8,000 \text { gal }) \text { of liquid waste per year from the PFP. }\end{array}$ & $\begin{array}{l}\text { This is the maximum projected annual volume from the PFP as described } \\
\text { in Appendix } H \text { of the TWRSO\&UP. }\end{array}$ \\
\hline
\end{tabular}


Table 2. Double-Shell Tank System Performance Requirements. (7 Sheets)

\begin{tabular}{|c|c|l|l|}
\hline Number & $\begin{array}{l}\text { Paragraph } \\
\text { Number }\end{array}$ & \multicolumn{1}{|c|}{ Requirement } & Basis \\
\hline 37. & $3.2 .3 .3 . \mathrm{a}$ & $\begin{array}{l}\text { S Plant Waste Volume. The system shall accept } \\
\text { up to 235,000 L (62,000 gal) of liquid waste per } \\
\text { year from S Plant. }\end{array}$ & $\begin{array}{l}\text { This is the maximum projected annual volume from S Plant (222-S) as } \\
\text { described in Appendix H of the TWRSO\&UP. }\end{array}$ \\
\hline 38. & $3.2 .3 .5 . \mathrm{d}$ & $\begin{array}{l}\text { Single-Shell Tank Waste Retrieval Volume, } \\
\text { Phase 1. Beginning in FY 2003, the system shall } \\
\text { be capable of accepting 30 ML (7.8 Mgal) of SST } \\
\text { waste through the end of Phase 1. }\end{array}$ & $\begin{array}{l}\text { This is the total projected volume from SST retrieval as described in } \\
\text { Appendix H of the TWRSO\&UP. }\end{array}$ \\
\hline 39. & $\begin{array}{l}\text { West Area Liquid Waste Annual Capacity. The } \\
\text { system shail be capable of receiving at least 15 ML } \\
\text { (4.0 Mgal) of liquid waste per year through } \\
\text { Phase 1. }\end{array}$ & $\begin{array}{l}\text { This value is the sum of the maximum yearly quantity of waste } \\
\text { transferred from each of the following facilities as listed in Appendix H } \\
\text { of the TWRSO\&UP: } \\
\text { 1) 222-S Laboratory } \\
\text { 2) SST Retrieval } \\
\text { 3) T-Plant } \\
\text { 4) Plutonium Finishing Plant } \\
\text { 5) Salt well pumping } \\
\text { The 9 year value is the duration of Phase 1. }\end{array}$ \\
\hline 40. & 3.2.3.5.a & $\begin{array}{l}\text { West Single-Shell Tank Salt Well Liquid Waste } \\
\text { Volume. The DST system shall be capable of } \\
\text { accepting 9.5 ML (2.5 Mgal) of dilute, non- } \\
\text { complexed (DN) and 3.4 ML (0.9 Mgal) of dilute, } \\
\text { complexed (DC) liquid from salt well pumping } \\
\text { from the SSTs over 4 years. }\end{array}$ & $\begin{array}{l}\text { These values are derived from the transfer schedule listed in Appendix H } \\
\text { of the TWRSO\&UU. }\end{array}$ \\
\hline
\end{tabular}

DST $=$ Double-shell tank

HLW $=$ High-level waste

LAW $=$ Low-activity waste

SST $=$ Single-shell tank

TBD $=$ To be determined

TWRSO\&UP = Tank Waste Remediation System Operation and Utilization Plan. 
HNF-2168

Revision 0

\subsection{REFERENCES}

Acree, C. D., 1998, Tank Waste Remediation System Mission Analysis Report, HNF-SD-WM-MAR-008, Rev. 0, Lockheed Martin Hanford Corporation, Richland, Washington.

Boston, H. L., 1997, Design Basis for Retrieval Equipment in Tanks AN-104 and AN-105, letter number LHMC-9758071 to A. M. Umek (September 15), Lockheed Martin Hanford Corporation, Richland, Washington.

Certa, P. J., C. M. McConville, L. W. Shelton, E. J. Slaathaug, 1996, Preliminary LowLevel Waste Feed Staging Plan, WHC-SD-WM-RPT-210, Rev. 0, Westinghouse Hanford Company, Richland, Washington.

Claghorn, R. D., J. D. Galbraith and T. B. Salzano, 1996, Alternatives Generation and Analysis for Phase 1 Intermediate Waste Feed Staging System Design Requirements, WHC-SD-TWR-AGA-001, Rev. 1, Westinghouse Hanford Company, Richland, Washington.

Claghorn, R. D., 1998, Decision Document, Waste Preparation Equipment for Phase I Source Tanks, (To be published), Numatec Hanford Corporation, Richland, Washington.

Claghorn, R. D., J. D. Galbraith, R. M. Marusich, T. B. Salzano, 1998, Alternatives Generation and Analysis for Waste Preparation Equipment: Phase I Source Tanks, HNF-SD-TWR-AGA-002, Rev. 0, Numatec Hanford Corporation, Richland, Washington.

Daling, P. M., 1996, Decision Document, Low-Level Waste Feed Staging Strategy, WHC-SD-WM-TI-788, Rev. 0, Westinghouse Hanford Company, Richland, Washington.

Galbraith, J. D., T. B. Salzano and J. R. Nicholson, 1996, Decision Document for Phase I Privatization Transfer System Needs, WHC-SD-WM-TI-750, Rev 0., Westinghouse Hanford Company, Richland, Washington.

Galbraith, J. D., and P. M. Daling (PNNL), 1997, Decision Document: Phase I Intermediate Waste Feed Staging System Design Requirements, HNF-SD-WM-TI-800, Rev. 0, Numatec Hanford Corporation, Richland, Washington.

Grenard, C. E., and R. D. Claghorn, 1998, System Specification for the Double-Shell Tank System, HNF-SD-WM-TRD-007, Rev. 0, Numatec Hanford Corporation, Richland, Washington. 
Hall, M. N., 1996, Life-Cycle Cost Analysis of Advanced Design Mixer Pump, WHC-SD-WM-CBA-001, Rev. 0, Westinghouse Hanford Company, Richland, Washington.

Kirkbride, R. A., G. K. Allen, P. J. Certa, A. F. Manuel, R. M. Orme, L. W. Shelton, E. J. Slaathaug, R. S. Wittman, and G. T. MacLean and D. L. Penwell (SESC), 1997, Tank Waste Remediation System Operation and Utilization Plan, HNF-SD-WM-SP-012, Rev. 0, Vol. I and II, Numatec Hanford Corporation, Richland, Washington.

Mulkey, C. H., 1997, Data Quality Objectives for Tank Farms Waste Compatibility Program, HNF-SD-WM-DQO-001, Rev 2, Lockheed Martin Hanford Corporation, Richland, Washington.

Swita, W. R., 1998, Tank Waste Remediation System Retrieval and Disposal Mission Initial Updated Baseline Summary, HNF-1946, Rev. 1, Lockheed Martin Hanford Corporation, Richland, Washington. 
HNF-2168

Revision 0

APPENDIX A

CALCULATION SHEETS

Performed by:

Checked by:

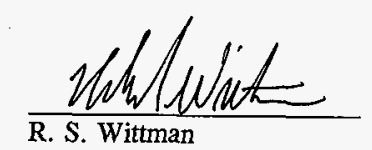

R. S. Wittman

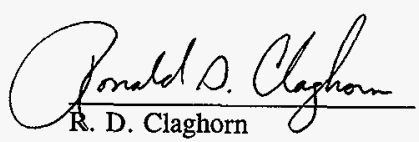

FEB 61998

Date

$7 e 66,1998$

A-1 
HNF-2168

Revision 0

This page intentionally left blank. 
HNF-2168

Revision 0

\section{APPENDIX A}

\section{CALCULATION SHEETS}

This appendix provides the raw data and the example calculations necessary to derive the performance requirements listed in the main body of this document. Sections A.1, A.2 and A.3 provide general information. Section A.4 is oriented to specific requirements.

\section{A.1 EXPLANATION OF TWRSO\&UP TRANSFER TABLE}

Table A-1 is taken from the Tank Waste Remediation System Operations and Utilization Plan (Kirkbride et al. 1997). It was generated using a computer program called the Hanford Tank Waste Operations Simulator (HTWOS). The HTWOS program was verified and validated by the Verification and Validation of the Tank Waste Remediation System Hanford Tank Waste Operations Simulator (Wittman 1997).

Table A-2 is a legend for the "from" and "to" information in Table A-1. Other information required to read Table A-1 is given below.

\section{A.1.1 Generic Transfers}

Most of the transfers in the table are simple tank to tank or waste source to tank transfers, but some are more complicated. The more elaborate transfers are described separately below. In any event, all transfers consist of the tank pumped from, the tank pumped to, the start date of the transfer, the end date of the transfer, the liquid volume transferred, and the solids volume transferred on a dry solids basis. The very small amount of solids transferred for some transfers is due to the assumption that a small fraction of the solids in a tank will be entrained in the liquid.

\section{A.1.2 Evaporator Runs}

Evaporator runs consist of three lines in the transfer table. The first line gives the transfer from the evaporator feed tank (AW-102) to 242-A, the second line gives the transfer from 242-A to the evaporator bottoms catch tank (AW-106), and the third line gives the transfer from 242-A to the condensate catch tank (defined as LERF in the model). 


\section{A.1.3 In-Line Dilution Transfers}

For some of the transfers in support of the LAW feed staging plan, DST contents will be retrieved and transferred using in-line dilution. In-line dilution consists of pumping (and mixing if desired) the DST contents as they exist and adding dilution/transfer water in the line. No water is added directly to the tank. Also if any dissolution/precipitation reactions are to occur they will occur in the line. Therefore, in order to be accurate, this transfer needed to be described in three steps: what is removed from the source tank, the amount of water added, and what is added to the destination tank.

The in-line dilution transfers can be found in the transfer table by examining the "To" column to find an entry that is "name of a DST"-PUMP. This entry is for the addition of the dilution water. The entry before this one gives the transfer out of the source tank and the entry after gives the transfer to the destination tank. For the some of the transfers one can see that the solids transferred out of the source tank do not equal the solids transferred into the destination tank. This is due to the dissolution/precipitation reactions that occur in-line. 
Table A-1. Projected Transfers Through October 2011. (26 sheets)

Note: This table is extracted from Table $\mathrm{H}-1$ of the Tank Waste Remediation System

Operations and Utilization Plan (Kirkbride et al. 1997)

\begin{tabular}{|c|c|c|c|c|c|c|c|c|c|}
\hline $\begin{array}{l}\text { Boot } \\
\text { Strap }\end{array}$ & $\begin{array}{l}\text { HLW } \\
\text { Flag }\end{array}$ & $\begin{array}{l}\text { LAW } \\
\text { Flag }\end{array}$ & From & To & $\begin{array}{l}\text { Start } \\
\text { Date }\end{array}$ & $\begin{array}{l}\text { End } \\
\text { Date }\end{array}$ & $\begin{array}{c}\text { Liquid } \\
\text { (gallons) }\end{array}$ & $\begin{array}{c}\text { Solid } \\
\text { (Gallons) }\end{array}$ & Notes \\
\hline & & & EVAPF & $A W-102$ & $12 / 5 / 93$ & $12 / 5 / 93$ & 28,000 & 0 & \\
\hline & & & ZNL87 & SY-102 & $4 / 4 / 94$ & $4 / 4 / 94$ & 4,000 & 0 & \\
\hline & & & ZNL87 & SY-102 & $4 / 4 / 94$ & $4 / 4 / 94$ & 3,000 & 0 & \\
\hline & & & WNW88 & SY-102 & $4 / 19 / 94$ & $4 / 19 / 94$ & 27,000 & 0 & . \\
\hline & & & AW-106 & AW-102 & $4 / 15 / 94$ & $4 / 19 / 94$ & 870,300 & 31 & \\
\hline & & & AW-102 & $242-A$ & $4 / 25 / 94$ & $5 / 11 / 94$ & $1,826,000$ & 64 & \\
\hline & & & 242-A & AW-106 & $4 / 25 / 94$ & $5 / 11 / 94$ & $1,223,000$ & 64 & \\
\hline & & & 242-A & LERF & $4 / 25 / 94$ & $5 / 11 / 94$ & 603,000 & 0 & \\
\hline & & & AW-106 & AW-102 & $5 / 15 / 94$ & $5 / 21 / 94$ & $1,222,000$ & 44 & \\
\hline & & & $\mathrm{AP}-103$ & AW-102 & $5 / 19 / 94$ & $5 / 25 / 94$ & $1,092,000$ & 37 & . \\
\hline & & & AW-106 & AW-102 & $6 / 5 / 94$ & $6 / 8 / 94$ & 680,000 & 56 & \\
\hline & & & AW-102 & $242-\mathrm{A}$ & $5 / 25 / 94$ & $6 / 15 / 94$ & $2,366,000$ & 83 & \\
\hline & & & $242-A$ & AW-106 & $5 / 25 / 94$ & $6 / 15 / 94$ & 968,800 & 83 & \\
\hline & & & $242-A$ & LERF & $5 / 25 / 94$ & $6 / 15 / 94$ & $1,397,000$ & 0 & \\
\hline & & & WATER & AW-102 & $6 / 16 / 94$ & $6 / 16 / 94$ & 28,000 & 0 & . \\
\hline & . & & AW-102 & $242-\mathrm{A}$ & $6 / 15 / 94$ & $6 / 21 / 94$ & 656,500 & 23 & \\
\hline & & & $242-A$ & $\mathrm{AW}-106$ & $6 / 15 / 94$ & $6 / 21 / 94$ & 379,900 & 23 & \\
\hline & & & $242-A$ & LERF & $6 / 15 / 94$ & $6 / 21 / 94$ & 276,600 & 아 & \\
\hline & & & AW-106 & $\mathrm{AW}-102$ & $6 / 25 / 94$ & $6 / 25 / 94$ & 52,998 & 2 & \\
\hline & & & AP-101 & AW-102 & $8 / 11 / 94$ & $8 / 15 / 94$ & 744,000 & 0 & \\
\hline & & & EVAPF & $\mathrm{AW}-102$ & $8 / 25 / 94$ & $8 / 25 / 94$ & 46,000 & 0 & \\
\hline & & & AP-107 & $\mathrm{AW}-102$ & $9 / 10 / 94$ & 9/15/94 & 900,000 & 0 & \\
\hline & & & $\mathrm{AW}-106$ & AN-106 & $9 / 19 / 94$ & 9/21/94 & 400,000 & 17 & \\
\hline & & & $\mathrm{AW}-102$ & $242-\mathrm{A}$ & $9 / 15 / 94$ & $9 / 30 / 94$ & $1,772,000$ & 61 & \\
\hline & & & $242-A$ & AW-106 & 9/15/94 & 9/30/94 & $1,240,000$ & 61 & . \\
\hline & & & $242-\mathrm{A}$ & LERF & $9 / 15 / 94$ & $9 / 30 / 94$ & 532,000 & 요 & \\
\hline & & & AP-107 & AW-102 & $10 / 1 / 94$ & $10 / 1 / 94$ & 157,300 & 0 & \\
\hline & & & AW-106 & AW-102 & $9 / 30 / 94$ & $10 / 4 / 94$ & 739,400 & 34 & \\
\hline & & & AW-106 & AW-102 & $10 / 5 / 94$ & $10 / 6 / 94$ & 213,000 & 10 & \\
\hline & & & AP-108 & AP-101 & $10 / 7 / 94$ & $10 / 8 / 94$ & 284,000 & 0 & \\
\hline & & & AP-101 & $\mathrm{AW}-102$ & $10 / 8 / 94$ & $10 / 10 / 94$ & 451,000 & 0 & \\
\hline & & & AP-108 & AW-102 & $10 / 15 / 94$ & $10 / 19 / 94$ & 814,400 & 0 & \\
\hline & & & AW-103 & AP-107 & $10 / 19 / 94$ & $10 / 19 / 94$ & 130,600 & 5 & - \\
\hline & & & AW-102 & $242-A$ & $10 / 25 / 94$ & $11 / 4 / 94$ & $2,347,000$ & 81 & \\
\hline & & & $242-A$ & AW-106 & $10 / 25 / 94$ & $11 / 4 / 94$ & 859,900 & 81 & \\
\hline & & & $242-A$ & LERF & $10 / 25 / 94$ & $11 / 4 / 94$ & $1,487,000$ & 0 & \\
\hline & & & AW-106 & AW-102 & $11 / 15 / 94$ & $11 / 21 / 94$ & $1,275,000$ & 46 & \\
\hline & & & AW-105 & AP-108 & $11 / 25 / 94$ & $11 / 27 / 94$ & 480,000 & 17. & \\
\hline & & & WATER & AW-102 & $11 / 30 / 94$ & $11 / 30 / 94$ & 35,000 & of & \\
\hline & & & AW-106 & AW-102 & $11 / 30 / 94$ & $11 / 30 / 94$ & 50,996 & 4 & \\
\hline & & & AW-105 & AP-108 & $12 / 1 / 94$ & $12 / 1 / 94$ & 170,700 & 6 & \\
\hline
\end{tabular}


HNF-2168

Revision 0

Table A-1. Projected Transfers Through October 2011. (26 sheets)

Note: This table is extracted from Table $\dot{\mathrm{H}}-1$ of the Tank Waste Remediation System

Operations and Utilization Plan (Kirkbride et al. 1997)

\begin{tabular}{|c|c|c|c|c|c|c|c|c|c|}
\hline $\begin{array}{l}\text { Boot } \\
\text { Strap }\end{array}$ & $\begin{array}{c}\text { HLW } \\
\text { Flag }\end{array}$ & $\begin{array}{c}\text { LAW } \\
\text { Flag } \\
\end{array}$ & From & To & $\begin{array}{l}\text { Start } \\
\text { Date } \\
\end{array}$ & $\begin{array}{l}\text { End } \\
\text { Date } \\
\end{array}$ & $\begin{array}{c}\text { Liquid } \\
\text { (gallons) }\end{array}$ & $\begin{array}{c}\begin{array}{c}\text { Solid } \\
\text { (Gallons) }\end{array} \\
\end{array}$ & Notes \\
\hline & & & $\mathrm{AW}-102$ & 242-A & $11 / 25 / 94$ & $12 / 6 / 94$ & $1,275,000$ & 46 & \\
\hline & & & $242-A$ & AW-106 & $11 / 25 / 94$ & $12 / 6 / 94$ & 501,700 & 46 & \\
\hline & & & $242-A$ & LERF & $11 / 25 / 94$ & $12 / 6 / 94$ & 773,300 & 0 & \\
\hline & & & AP-108 & AP-101 & $1 / 20 / 95$ & $1 / 23 / 95$ & 650,700 & 21 & \\
\hline & & & AP-101 & AP-107 & $1 / 22 / 95$ & $1 / 25 / 95$ & 774,600 & 19 & \\
\hline & & & WATER & SY-102 & $2 / 22 / 95$ & $2 / 22 / 95$ & 8,000 & 0 & \\
\hline & & & SPN87 & SY-102 & $2 / 22 / 95$ & $2 / 22 / 95$ & 22,000 & 0 & \\
\hline & & & $\mathrm{AP}-106$ & AW-102 & $3 / 28 / 95$ & $3 / 28 / 95$ & 83,000 & 0 & \\
\hline & & & EVAPF & AW-102 & $4 / 1 / 95$ & $4 / 1 / 95$ & 52,000 & 0 & \\
\hline & & & EVAPF & SY-102 & 4/7/95 & $4 / 7 / 95$ & 15,000 & 0 & \\
\hline & & & AP-106 & AP-108 & $5 / 4 / 95$ & $5 / 8 / 95$ & 934,000 & 0 & \\
\hline & & & AP-107 & AW-102 & $5 / 19 / 95$ & $5 / 20 / 95$ & 371,000 & 9 & \\
\hline & & & AP-108 & AW-102 & $5 / 23 / 95$ & $5 / 24 / 95$ & 372,000 & 0 & \\
\hline & & & WATER & $A W-102$ & $6 / 1 / 95$ & $6 / 1 / 95$ & 13,000 & 0 & \\
\hline & & & AW-106 & AW-102 & $6 / 5 / 95$ & $6 / 7 / 95$ & 443,000 & 17. & \\
\hline & & & AP-107 & AW-102 & $6 / 12 / 95$ & $6 / 14 / 95$ & 534,200 & 13 & \\
\hline & & & AP-108 & AW-102 & $6 / 13 / 95$ & $6 / 15 / 95$ & 559,000 & 1 & \\
\hline & & & AW-102 & $242-A$ & $6 / 15 / 95$ & $6 / 26 / 95$ & $2,513,000$ & 14 & \\
\hline & & & $242+\mathrm{A}$ & AW-106 & $6 / 15 / 95$ & $6 / 26 / 95$ & $1,180,000$ & 14 & \\
\hline & & & 242-A & LERF & $6 / 15 / 95$ & $6 / 26 / 95$ & $1,333,000$ & 0 & \\
\hline & & & AW-106 & $\mathrm{AW}-102$ & $6 / 30 / 95$ & $7 / 2 / 95$ & 424,000 & 16 & \\
\hline & & & AW-106 & AW-102 & $7 / 5 / 95$ & $7 / 8 / 95$ & 764,000 & 28 & - \\
\hline & & & AW-102 & $242-A$ & $7 / 15 / 95$ & $7 / 20 / 95$ & $1,188,000$ & 44 & \\
\hline & & & $242-A$ & AW-106 & $7 / 15 / 95$ & $7 / 20 / 95$ & 367,500 & 44 & \\
\hline & & & $242-\mathrm{A}$ & LERF & $7 / 15 / 95$ & $7 / 20 / 95$ & 820,400 & 0 & \\
\hline & & & AW-106 & AW-102 & $7 / 30 / 95$ & $7 / 30 / 95$ & 33,999 & 1 & \\
\hline & & & SY-102 & & $8 / 2 / 95$ & $8 / 4 / 95$ & 430,000 & 15 & $\begin{array}{l}\text { Out-of-system } \\
\text { Volume } \\
\text { Reconciliation }\end{array}$ \\
\hline & & & WNW88 & $S Y-102$ & $8 / 12 / 95$ & $8 / 13 / 95$ & 12,000 & 0 & $\cdot$ \\
\hline & & & WATER & SY-102 & $8 / 14 / 95$ & $8 / 14 / 95$ & 6,000 & 0 & . \\
\hline & & & AP-105 & AP-101 & $8 / 27 / 95$ & $8 / 30 / 95$ & 660,000 & 29 & \\
\hline & & & PXTCO & AW-105 & $11 / 7 / 95$ & $11 / 8 / 95$ & 334,000 & 요 & \\
\hline & & & WATER & AW-105 & $11 / 9 / 95$ & $11 / 9 / 95$ & 19,000 & 0 & \\
\hline & & & AW-105 & & $11 / 13 / 95$ & $11 / 14 / 95$ & 330,000 & 11 & $\begin{array}{l}\text { Out-of-system } \\
\text { Yolume } \\
\text { Reconciliation }\end{array}$ \\
\hline & & & BPTCO & AP-106 & $2 / 8 / 96$ & $2 / 8 / 96$ & 14,000 & 0 & . \\
\hline & & & WATER & AP-106 & $2 / 16 / 96$ & $2 / 16 / 96$ & 3,000 & 0 & \\
\hline & & & $34 \mathrm{~L} 87$ & AP-106 & $2 / 16 / 96$ & $2 / 16 / 96$ & 28,000 & 0 & \\
\hline & & & EVAPF & AW-102 & $3 / 1 / 96$ & $3 / 1 / 96$ & 67,000 & 0 & \\
\hline & & & AW-106 & AP-101 & $3 / 4 / 96$ & $3 / 5 / 96$ & 306,000 & 13 & \\
\hline
\end{tabular}


Table A-1. Projected Transfers Through October 2011. (26 sheets)

Note: This table is extracted from Table H-1 of the Tank Waste Remediation System

Operations and Utilization Plan (Kirkbride et al. 1997)

\begin{tabular}{|c|c|c|c|c|c|c|c|c|c|}
\hline $\begin{array}{l}\text { Boot } \\
\text { Strap }\end{array}$ & $\begin{array}{l}\text { HLW } \\
\text { Flag }\end{array}$ & $\begin{array}{c}\text { LAW } \\
\text { Flag } \\
\end{array}$ & From & To & $\begin{array}{l}\text { Start } \\
\text { Date }\end{array}$ & $\begin{array}{l}\text { End } \\
\text { Date }\end{array}$ & $\begin{array}{c}\text { Liquid } \\
\text { (gallons) }\end{array}$ & $\begin{array}{c}\text { Solid } \\
\text { (Gallons) }\end{array}$ & Notes \\
\hline & & & UNKN & AP-106 & $3 / 18 / 96$ & $3 / 18 / 96$ & 0 & 0 & \\
\hline & & & WATER & AP-106 & $3 / 18 / 96$ & $3 / 18 / 96$ & 28,000 & 0 & \\
\hline & & & BPTCO & AP-106 & $3 / 20 / 96$ & $3 / 20 / 96$ & 26,000 & 0 & $\cdot$ \\
\hline & & & EVAPF & AW-106 & $4 / 1 / 96$ & $4 / 1 / 96$ & 14,000 & 0 & \\
\hline & & & EVAPF & AW-102 & $4 / 1 / 96$ & $4 / 1 / 96$ & 22,000 & 0 & \\
\hline & & & WNE88 & SY-102 & $3 / 20 / 96$ & $4 / 12 / 96$ & 169,000 & 0 & \\
\hline & & & WATER & SY-102 & $4 / 13 / 96$ & $4 / 13 / 96$ & 49,000 & 0 & \\
\hline & & & SPN87 & AP-106 & $4 / 17 / 96$ & $4 / 17 / 96$ & 11,000 & 0 & \\
\hline & & & SPN87 & AP-106 & $4 / 17 / 96$ & $4 / 17 / 96$ & 11,000 & 0 & \\
\hline & & & WATER & AP-108 & $4 / 19 / 96$ & $4 / 19 / 96$ & 3,000 & 0 & . \\
\hline & & & BPTCO & AP-108 & $4 / 19 / 96$ & $4 / 19 / 96$ & 14,000 & 0 & \\
\hline & & & AW-106 & AW-102 & $5 / 6 / 96$ & $5 / 6 / 96$ & 28,999 & 1 & \\
\hline & & & AP-104 & AW-102 & $5 / 6 / 96$ & $5 / 10 / 96$ & 812,000 & 0 & \\
\hline & & & AW-102 & $242-A$ & $5 / 15 / 96$ & $5 / 19 / 96$ & 964,300 & 33 & \\
\hline & & & $242-\mathrm{A}$ & AW-106 & $5 / 15 / 96$ & $5 / 19 / 96$ & 124,600 & 33 & \\
\hline & & & $242-\mathrm{A}$ & LERF & $5 / 15 / 96$ & $5 / 19 / 96$ & 839,700 & 0 & \\
\hline & & & EVAPF & AW-102 & $5 / 30 / 96$ & $5 / 30 / 96$ & 3,000 & 0 & \\
\hline & & & AW-106 & AW-102 & $5 / 30 / 96$ & $5 / 30 / 96$ & 61,997 & 3 & .. \\
\hline & & & TAL88 & AP-106 & $6 / 6 / 96$ & $6 / 6 / 96$ & 10,000 & 0 & \\
\hline & & & PXTCO & AW-105 & $6 / 30 / 96$ & $6 / 30 / 96$ & 95,000 & 0 & \\
\hline & & & WATER & AP-105 & $7 / 1 / 96$ & $7 / 1 / 96$ & 1,000 & 0 & \\
\hline & & & WATER & $A Y-102$ & $8 / 15 / 96$ & $8 / 15 / 96$ & 27,000 & 0 & \\
\hline & & & AN-101 & AP-105 & $8 / 16 / 96$ & $8 / 20 / 96$ & 928,800 & 0 & \\
\hline & & & AW-106 & AP-101 & $1 / 9 / 97$ & $1 / 9 / 97$ & 69,511 & 3 & \\
\hline & & & AN-106 & AP-108 & $1 / 16 / 97$ & $1 / 16 / 97$ & 177,000 & 7 & \\
\hline & & & AP-105 & AW-102 & $2 / 10 / 97$ & $2 / 14 / 97$ & 942,000 & 42 & \\
\hline & & & AW-102 & 242-A & $2 / 26 / 97$ & $3 / 2 / 97$ & 977,500 & 43 & \\
\hline & & & $242-A$ & AW-106 & $2 / 26 / 97$ & $3 / 2 / 97$ & 576,400 & 43 & \\
\hline & & & 242-A & LERF & $2 / 26 / 97$ & $3 / 2 / 97$ & 401,100 & 0 & \\
\hline & & & AY-101 & AP-108 & $3 / 3 / 97$ & $3 / 7 / 97$ & 809,000 & 30 & \\
\hline & & & AW-106 & AP-105 & $4 / 3 / 97$ & $4 / 5 / 97$ & 576,200 & 29 & \\
\hline & & & AN-106 & AP-108 & $7 / 1 / 97$ & $7 / 7 / 97$ & 194,000 & 7 & \\
\hline & & & AP-108 & AW-102 & $7 / 3 / 97$ & $7 / 10 / 97$ & $1,198,000$ & 43 & \\
\hline & & & $\mathrm{AW}-102$ & $242-A$ & $.7 / 5 / 97$ & $7 / 10 / 97$ & $1,198,000$ & 40 & \\
\hline & & & 242-A & AW-106 & $7 / 5 / 97$ & 7/10/97 & 346,700 & 40 & \\
\hline & & & $242-A$ & LERF & $7 / 5 / 97$ & $7 / 10 / 97$ & 851,300 & of & \\
\hline & & & ZNL87 & SY-102 & $7 / 31 / 97$ & $7 / 31 / 97$ & 2,000 & 0 & \\
\hline & & & WATER & SY-102 & $7 / 31 / 97$ & $7 / 31 / 97$ & 62,000 & 0 & \\
\hline & & & WNW88 & SY-102 & $7 / 31 / 97$ & $8 / 1 / 97$ & 223,000 & 0 & \\
\hline & & & AW-106 & AN-106 & $8 / 5 / 97$ & $8 / 6 / 97$ & 346,400 & 16 & \\
\hline & & & $5 Y-102$ & AP-107 & $8 / 15 / 97$ & $8 / 17 / 97$ & 520,000 & 20 & · \\
\hline
\end{tabular}


HNF-2168

Revision 0

Table A-1. Projected Transfers Through October 2011. (26 sheets)

Note: This table is extracted from Table H-1 of the Tank Waste Remediation System Operations and Utilization Plan (Kirkbride et al. 1997)

\begin{tabular}{|c|c|c|c|c|c|c|c|c|c|}
\hline $\begin{array}{l}\text { Boot } \\
\text { Strap }\end{array}$ & $\begin{array}{c}\text { HLW } \\
\text { Flag }\end{array}$ & $\begin{array}{c}\text { LAW } \\
\text { Flag }\end{array}$ & From & To & $\begin{array}{l}\text { Start } \\
\text { Date }\end{array}$ & $\begin{array}{l}\text { End } \\
\text { Date }\end{array}$ & $\begin{array}{c}\text { Liquid } \\
\text { (gallons) }\end{array}$ & $\begin{array}{c}\text { Solid } \\
\text { (Gallons) }\end{array}$ & - Notes \\
\hline & & & $\begin{array}{l}\text { WASH- } \\
\text { CAUSTIC }\end{array}$ & AN-107 & $10 / 2 / 97$ & $10 / 2 / 97$ & 66,000 & 0 & \\
\hline & & & AY-102 & AP-106 & $10 / 7 / 97$ & $10 / 7 / 97$ & 35,999 & 1 & \\
\hline & & & WATER & AY-102 & $10 / 8 / 97$ & $10 / 8 / 97$ & 176,000 & 0 & \\
\hline & & & ZNL87 & SY-102 & $11 / 30 / 97$ & $11 / 30 / 97$ & 4,000 & 0 & \\
\hline & & & SPN87 & SY-102 & $11 / 30 / 97$ & $11 / 30 / 97$ & 7,000 & 0 & \\
\hline & & & WATER & SY-102 & $11 / 30 / 97$ & $11 / 30 / 97$ & 112,000 & 0 & . \\
\hline & & & WNW88 & SY-102 & $11 / 30 / 97$ & $12 / 2 / 97$ & 422,000 & 0 & \\
\hline & & & TNS88 & AP-106 & $12 / 5 / 97$ & $12 / 5 / 97$ & 1,500 & 500 & \\
\hline & & & PXTCO & $\mathrm{AP}-106$ & $12 / 5 / 97$ & $12 / 5 / 97$ & 10,000 & 0 & \\
\hline & & & TAL88 & AP-106 & $12 / 5 / 97$ & $12 / 5 / 97$ & 20,000 & 0 & \\
\hline & & & WESF & AP-106 & $12 / 5 / 97$ & $12 / 5 / 97$ & 25,000 & 0 & . \\
\hline & & & SPN87. & AP-106 & $12 / 5 / 97$ & $12 / 5 / 97$ & 25,000 & 0 & \\
\hline & & & $34 \mathbf{L} 87$ & AP-106 & $12 / 5 / 97$ & $12 / 5 / 97$ & 67,000 & 0 & \\
\hline & & & WATER & AP-106 & $12 / 5 / 97$ & $12 / 5 / 97$ & 109,000 & 0 & \\
\hline & & & BPTCO & AP-106 & $12 / 5 / 97$ & $12 / 5 / 97$ & 136,000 & 0 & \\
\hline & & & EVAPF & AP-106 & $12 / 5 / 97$ & $12 / 5 / 97$ & 150,000 & 0 & \\
\hline & & & WNE88 & AP-106 & $12 / 5 / 97$ & $12 / 6 / 97$ & 204,000 & 0 & \\
\hline & & & SY-102 & AP-107 & $12 / 15 / 97$ & $12 / 17 / 97$ & 520,000 & 21 & \\
\hline & & & AP-106 & AP-104 & $12 / 20 / 97$ & $12 / 25 / 97$ & 996,800 & 35 & \\
\hline & & & SPN87 & SY-102 & $2 / 28 / 98$ & $2 / 28 / 98$ & 6,000 & 0 & \\
\hline & & & WATER & SY-102 & $2 / 28 / 98$ & $2 / 28 / 98$ & 103,000 & 0 & \\
\hline & & & WNW88 & SY-102 & $2 / 28 / 98$ & $3 / 2 / 98$ & 410,000 & 0 & \\
\hline & & & AP-104 & AW-102 & $3 / 1 / 98$ & $3 / 6 / 98$ & 995,300 & 34 & \\
\hline & & & SY-102 & AP-104 & $3 / 15 / 98$ & $3 / 17 / 98$ & 520,000 & 21 & \\
\hline & & & AW-102 & $242-\mathrm{A}$ & $4 / 5 / 98$ & \begin{tabular}{|l|}
$4 / 9 / 98$ \\
\end{tabular} & 995,300 & 35 & \\
\hline & & & $242-\mathrm{A}$ & AW-106 & $4 / 5 / 98$ & $4 / 9 / 98$ & 168,500 & 35 & \\
\hline & & & $242-\mathrm{A}$ & LERF & $4 / 5 / 98$ & $4 / 9 / 98$ & 826,800 & 0 & \\
\hline & & & WATER & AP-107 & $4 / 10 / 98$ & $4 / 10 / 98$ & 70,000 & 0 & \\
\hline & & & AP-107 & AW-102 & $4 / 25 / 98$ & $5 / 7 / 98$ & $1,104,000$ & 40 & i \\
\hline & & & AW-102 & 242-A & $5 / 5 / 98$ & $5 / 10 / 98$ & $1,104,000$ & 39 & \\
\hline & & & $242-\mathrm{A}$ & AW-106 & $5 / 5 / 98$ & $5 / 10 / 98$ & 441,000 & 39 & \\
\hline & & & $242-\mathrm{A}$ & LERF & $5 / 5 / 98$ & $5 / 10 / 98$ & 663,000 & 0 & \\
\hline & & & SPN87 & SY-102 & $5 / 31 / 98$ & $5 / 31 / 98$ & 6,000 & 0 & \\
\hline & & & WCW88 & SY-102 & $5 / 31 / 98$ & $5 / 31 / 98$ & 38,000 & 0 & $\cdot$ \\
\hline & & & WATER & SY-102 & $5 / 31 / 98$ & $5 / 31 / 98$ & 119,000 & 0 & \\
\hline & & & WNW88 & SY-102 & $5 / 31 / 98$ & $6 / 2 / 98$ & 435,000 & 0 & \\
\hline & & & AW-106 & AP-105 & $6 / 3 / 98$ & $6 / 4 / 98$ & 341,000 & 16 & \\
\hline & & & SY-102 & AP-104 & $6 / 15 / 98$ & $6 / 17 / 98$ & 520,000 & 21 & \\
\hline
\end{tabular}


HNF-2168

Revision 0

Table A-1. Projected Transfers Through October 2011. (26 sheets)

Note: This table is extracted from Table $\mathrm{H}-1$ of the Tank Waste Remediation System

Operations and Utilization Plan (Kirkbride et al. 1997)

\begin{tabular}{|c|c|c|c|c|c|c|c|c|c|}
\hline $\begin{array}{l}\text { Boot } \\
\text { Strap }\end{array}$ & $\begin{array}{c}\text { HLW } \\
\text { Flag }\end{array}$ & $\begin{array}{l}\text { LAW } \\
\text { Flag }\end{array}$ & From & To & $\begin{array}{l}\text { Start } \\
\text { Date }\end{array}$ & $\begin{array}{l}\text { End } \\
\text { Date }\end{array}$ & $\begin{array}{c}\text { Liquid } \\
\text { (gallons) }\end{array}$ & $\begin{array}{c}\text { Solid } \\
\text { (Gallons) }\end{array}$ & Notes \\
\hline & $\mathrm{Y}$ & & AY-102 & C-106 & $7 / 1 / 98$ & $7 / 5 / 98$ & 920,700 & 31 & $\begin{array}{l}\text { Sluice "Water" } \\
\text { for C-106 } \\
\text { Retrieval - } \\
\text { Lumped (See } \\
7 / 15 / 98 \text { ) }\end{array}$ \\
\hline & & & AP-101 & AW-103 & $8 / 6 / 98$ & $8 / 9 / 98$ & 625,000 & 27 & . \\
\hline & & & SPN87 & SY-102 & $8 / 31 / 98$ & $8 / 31 / 98$ & 6,000 & 0. & \\
\hline & & & WCW88 & SY-102 & $8 / 31 / 98$ & $8 / 31 / 98$ & 34,000 & 0 & \\
\hline & & & WATER & SY-102 & $8 / 31 / 98$ & $8 / 31 / 98$ & 104,000 & 0 & \\
\hline & & & WNW88 & SY-102 & $8 / 31 / 98$ & 9/1/98 & 385,000 & 0 & \\
\hline & & & SY-102 & AP-107 & $9 / 15 / 98$ & 9/17/98 & 520,000 & 21 & \\
\hline & & & WATER & AP-104 & $9 / 18 / 98$ & $9 / 18 / 98$ & 70,000 & 0 & . \\
\hline & & & AW-104 & AP-107 & $10 / 10 / 98$ & $10 / 12 / 98$ & 540,000 & 18 & \\
\hline & & & EVAPF & ÁW-102 & $10 / 13 / 98$ & $10 / 14 / 98$ & 195,000 & 0 & \\
\hline & & & AW-104 & AP-104 & $10 / 14 / 98$ & $10 / 14 / 98$ & 164,000 & 6 & \\
\hline & & & AP-101 & AW-104 & $10 / 21 / 98$ & $10 / 23 / 98$ & 460,000 & 20 & \\
\hline & & $\dot{1}$ & AW-106 & AP-101 & $10 / 27 / 98$ & $10 / 28 / 98$ & 289,000 & 13 & \\
\hline & & & AW-102 & AW-106 & $10 / 28 / 98$ & $10 / 28 / 98$ & 150,000 & 6 & \\
\hline & & & AW-106 & $A W-102$ & $11 / 6 / 98$ & $11 / 6 / 98$ & 150,000 & 6 & \\
\hline & & & AP-104 & $\mathrm{AW}-102$ & $10 / 3 / 98$ & $11 / 10 / 98$ & $1,110,000$ & 42 & $\cdot$ \\
\hline & & & AW-102 & 242-A & $11 / 5 / 98$ & $11 / 10 / 98$ & $1,195,000$ & 46 & \\
\hline & & & 242-A & AW-106 & $11 / 5 / 98$ & $11 / 10 / 98$ & 585,000 & 46 & \\
\hline & & & $242-\mathrm{A}$ & LERF & $11 / 5 / 98$ & $11 / 10 / 98$ & 610,000 & 0 & \\
\hline & $\mathbf{Y}$ & & C-106 & $A Y-102$ & $7 / 15 / 98$ & $11 / 26 / 98$ & 923,500 & 17,315 & $\begin{array}{l}\text { C-106 Retrieval } \\
\text { (Project W-320) }\end{array}$ \\
\hline & & & WCW88 & SY-102 & $11 / 30 / 98$ & $11 / 30 / 98$ & 14,000 & 0 & \\
\hline & & & SPN87 & SY-102 & $11 / 30 / 98$ & $11 / 30 / 98$ & 7,000 & 0 & \\
\hline & & & ZNL87 & SY-102 & $11 / 30 / 98$ & $11 / 30 / 98$ & 4,000 & 0 & \\
\hline & & & WATER & SY-102 & $11 / 30 / 98$ & $11 / 30 / 98$ & 98,000 & 0 & \\
\hline & & & WNW88 & SY-102 & $11 / 30 / 98$ & $12 / 1 / 98$ & 357,000 & 0 & \\
\hline & & & AW-106 & AP-101 & $12 / 1 / 98$ & $12 / 3 / 98$ & 563,900 & 25 & \\
\hline & & & SY-102 & AP-104 & $12 / 15 / 98$ & $12 / 17 / 98$ & 520,000 & 21 & \\
\hline & & & PXTCO & AP-106 & $12 / 31 / 98$ & $12 / 31 / 98$ & 5,000 & 0 & \\
\hline & & & TNS88 & AP-106 & $12 / 31 / 98$ & $12 / 31 / 98$ & 750 & 250 & \\
\hline & & & WESF & AP-106 & $12 / 31 / 98$ & $12 / 31 / 98$ & 5,000 & 0 & \\
\hline & & & TAL88 & AP-106 & $12 / 31 / 98$ & $12 / 31 / 98$ & 20,000 & 0 & \\
\hline & & & BPTCO & AP-106 & $12 / 31 / 98$ & $12 / 31 / 98$ & 44,000 & 0 & \\
\hline & & & $34 \mathrm{~L} 87$ & AP-106 & $12 / 31 / 98$ & $12 / 31 / 98$ & 60,000 & 0 & \\
\hline & & & WATER & AP-106 & $12 / 31 / 98$ & $12 / 31 / 98$ & 76,000 & 0 & \\
\hline & & & EVAPF & AP-106 & $12 / 31 / 98$ & $12 / 31 / 98$ & 130,000 & 0 & \\
\hline & & & WNE88 & AP-106 & $12 / 31 / 98$ & $1 / 1 / 99$ & 186,000 & 0 & \\
\hline & & & AP-106 & A.P-104 & $1 / 15 / 99$ & $1 / 16 / 99$ & 385,000 & 14 & \\
\hline
\end{tabular}


Table A-1. Projected Transfers Through October 2011. (26 sheets) Note: This table is extracted from Table H-1 of the Tank Waste Remediation System Operations and Utilization Plan (Kirkbride et al. 1997)

\begin{tabular}{|c|c|c|c|c|c|c|c|c|c|}
\hline $\begin{array}{l}\text { Boot } \\
\text { Strap }\end{array}$ & $\begin{array}{l}\text { HLW } \\
\text { Flag }\end{array}$ & $\begin{array}{l}\text { LAW } \\
\text { Flag }\end{array}$ & From & To & $\begin{array}{l}\text { Start } \\
\text { Date } \\
\end{array}$ & $\begin{array}{l}\text { End } \\
\text { Date }\end{array}$ & $\begin{array}{c}\text { Liquid } \\
\text { (gallons) }\end{array}$ & $\begin{array}{c}\text { Solid } \\
\text { (Galions) }\end{array}$ & Notes \\
\hline & & & WATER & AP-107 & $1 / 31 / 99$ & $1 / 31 / 99$ & 35,000 & 0 & \\
\hline & & & WATER & AN-101 & $2 / 15 / 99$ & $2 / 16 / 99$ & 194,000 & 0 & \\
\hline & & & WCE88 & AN-101 & $2 / 15 / 99$ & $2 / 18 / 99$ & 775,000 & 0 & \\
\hline & & & SPN87 & SY-102 & $2 / 28 / 99$ & $2 / 28 / 99$ & 6,000 & 0 & \\
\hline & & & WATER & SY-102 & $2 / 28 / 99$ & $2 / 28 / 99$ & 103,000 & 0 & \\
\hline & & & WCW88 & SY-102 & $2 / 28 / 99$ & $2 / 28 / 99$ & 135,000 & 0 & \\
\hline & & & WNW88 & SY-102 & $2 / 28 / 99$ & $3 / 1 / 99$ & 275,000 & 0 & \\
\hline & & & AN-101 & AP-103 & $3 / 2 / 99$ & $3 / 7 / 99$ & $1,024,000$ & 0 & \\
\hline & . & & AP-107 & AW-102 & $2 / 15 / 99$ & $3 / 9 / 99$ & $1,095,000$ & 40 & \\
\hline & & & AW-102 & $242-A$ & $3 / 5 / 99$ & $3 / 10 / 99$ & $1,130,000$ & 36 & \\
\hline & & & 242-A & AW-106 & $3 / 5 / 99$ & $3 / 10 / 99$ & 360,900 & 36 & \\
\hline & & & $242-\mathrm{A}$ & LERF & $3 / 5 / 99$ & $3 / 10 / 99$ & 769,000 & 0 & \\
\hline & & & SY-102 & AP-107 & $3 / 15 / 99$ & $3 / 17 / 99$ & 520,000 & 21 & \\
\hline & & & AW-106 & AP-101 & $4 / 3 / 99$ & $4 / 4 / 99$ & 224,000 & 10 & \\
\hline & & & AW-106 & AW-104 & $4 / 5 / 99$ & 4/5/99 & 136,800 & 6 & \\
\hline & & & SPN87 & SY-102 & $5 / 31 / 99$ & $5 / 31 / 99$ & 6,000 & 0 & : \\
\hline & & & WATER & SY-102 & $5 / 31 / 99$ & $5 / 31 / 99$ & 107,000 & 0 & . \\
\hline & & & WNW88 & SY-102 & $5 / 31 / 99$ & $5 / 31 / 99$ & 180,000 & 0 & \\
\hline & & & WCW88 & SY-102 & $5 / 31 / 99$ & $.6 / 1 / 99$ & 249,000 & 0 & \\
\hline & & & SY-102 & AP-107 & $6 / 15 / 99$ & $6 / 17 / 99$ & 520,000 & 22 & \\
\hline & & & AP-103 & AW-102 & $7 / 1 / 99$ & $7 / 8 / 99$ & $1,017,000$ & 44 & . \\
\hline & & & $\mathrm{AW}-102$ & 242-A & $7 / 5 / 99$ & 7/9/99 & 979,200 & 36 & \\
\hline & & & $242-A$ & AW-106 & $7 / 5 / 99$ & $7 / 9 / 99$ & 891,000 & 36 & \\
\hline & & & $242-A$ & LERF & $7 / 5 / 99$ & 7/9/99 & 88,202 & 0 & \\
\hline & & & AW-106 & AN-106 & $7 / 20 / 99$ & $7 / 23 / 99$ & 748,000 & 33 & \\
\hline & & & AW-106 & AP-108 & $7 / 25 / 99$ & $7 / 25 / 99$ & 143,000 & 6 & \\
\hline & & & WATER & AP-104 & $8 / 26 / 99$ & $8 / 26 / 99$ & 35,000 & 0 & \\
\hline & & & SPN87 & SY-102 & $8 / 31 / 99$ & $8 / 31 / 99$ & 6,000 & 0 & \\
\hline$\dot{-}$ & & & WATER & SY-102 & $8 / 31 / 99$ & $8 / 31 / 99$ & 98,000 & 0 & \\
\hline & & & WCW88 & SY-102 & $8 / 31 / 99$ & 9/1/99 & 188,000 & 0 & \\
\hline & & & WNW88 & SY-102 & $8 / 31 / 99$ & 9/1/99 & 206,000 & 0 &. \\
\hline & & & EVAPF & AW-102 & $9 / 7 / 99$ & 9/7/99 & 70,000 & 0 & \\
\hline & & & AW-102 & AW-106 & $9 / 22 / 99$ & $9 / 23 / 99$ & 300,000 & 12 & \\
\hline & & & SY-102 & AP-104 & 9/15/99 & $9 / 27 / 99$ & 520,000 & 22 & \\
\hline$Y$ & & $Y$ & AP-104 & AW-102 & $9 / 10 / 99$ & 9/29/99 & $1,104,000$ & 41 & Empty 4AP \\
\hline & & & $A W-106$ & AW-102 & $10 / 6 / 99$ & $10 / 7 / 99$ & 300,000 & 13 & \\
\hline$Y$ & & $Y$ & AP-102 & AP-103 & 10/3/99 & $10 / 8 / 99$ & $1,070,000$ & 0 & Empty $2 \mathrm{AP}$ \\
\hline & & & AW-102 & $242-\mathrm{A}$ & $10 / 5 / 99$ & $10 / 10 / 99$ & $1,139,000$ & 45 & \\
\hline & & & $242-A$ & AW-106 & $10 / 5 / 99$ & $10 / 10 / 99$ & 432,200 & 45 & \\
\hline & & & 242-A & LERF & $10 / 5 / 99$ & $10 / 10 / 99$ & 706,800 & 0 & \\
\hline & & & AW-106 & AW-104 & $10 / 12 / 99$ & $10 / 12 / 99$ & 111,000 & 5 & \\
\hline
\end{tabular}


Table A-1. Projected Transfers Through October 2011. (26 sheets)

Note: This table is extracted from Table H-1 of the Tank Waste Remediation System

Operations and Utilization Plan (Kirkbride et al. 1997)

\begin{tabular}{|c|c|c|c|c|c|c|c|c|c|}
\hline $\begin{array}{l}\text { Boot } \\
\text { Strap }\end{array}$ & $\begin{array}{l}\text { HLW } \\
\text { Flag }\end{array}$ & $\begin{array}{c}\text { LAW } \\
\text { Flag }\end{array}$ & From & To & $\begin{array}{l}\text { Start } \\
\text { Date }\end{array}$ & $\begin{array}{l}\text { End } \\
\text { Date }\end{array}$ & $\begin{array}{c}\text { Liquid } \\
\text { (gallons) }\end{array}$ & $\begin{array}{c}\text { Solid } \\
\text { (Gallons) }\end{array}$ & Notes \\
\hline & & & AW-106 & AN-101 & $10 / 13 / 99$ & $10 / 14 / 99$ & 321,000 & 16 & \\
\hline & & & WATER & AP-107 & $10 / 17 / 99$ & $10 / 17 / 99$ & 70,000 & 0 & \\
\hline & & & $A P-107$ & AW-102 & $11 / 1 / 99$ & $11 / 8 / 99$ & $1,110,000$ & 43 & \\
\hline & & & AW-102 & $242-A$ & $11 / 5 / 99$ & $11 / 10 / 99$ & $1,110,000$ & 34 & \\
\hline & & & $242-A$ & $A W-106$ & $11 / 5 / 99$ & $11 / 10 / 99$ & 732,200 & 34 & $\cdot$ \\
\hline & & & $242-\mathrm{A}$ & LERF & $11 / 5 / 99$ & $11 / 10 / 99$ & 377,700 & 0 & . \\
\hline & & & AW-106 & AN-101 & $12 / 12 / 99$ & $12 / 15 / 99$ & 732,200 & 33 & \\
\hline & & & SPN87 & SY-102 & $12 / 31 / 99$ & $12 / 31 / 99$ & 13,000 & 0 & \\
\hline & & & ZNL.87 & SY-102 & $12 / 31 / 99$ & $12 / 31 / 99$ & 4,000 & 0 & \\
\hline & & & WATER & SY-102 & $12 / 31 / 99$ & $12 / 31 / 99$ & 92,000 & 0 & . \\
\hline & & & WCW88 & SY-102 & $12 / 31 / 99$ & $12 / 31 / 99$ & 147,000 & 0 & \\
\hline & & & WNW88 & SY-102 & $12 / 31 / 99$ & $1 / 1 / 00$ & 192,000 & 0 & \\
\hline & & & SY-102 & AP-104 & $1 / 15 / 00$ & $1 / 17 / 00$ & 520,000 & 22 & \\
\hline & & & WATER & AP-104 & $6 / 17 / 00$ & $6 / 17 / 00$ & 70,000 & 0 & \\
\hline & & & AP-104 & AW-102 & $7 / 2 / 00$ & $7 / 8 / 00$ & $1,110,000$ & 43 & \\
\hline & & & AW-102 & $242-\mathrm{A}$ & $7 / 6 / 00$ & $7 / 11 / 00$ & $1,145,000$ & 36 & \\
\hline & & & 242-A & AW-106 & $7 / 6 / 00$ & $7 / 11 / 00$ & 778,300 & 36 & \\
\hline & & & 242-A & LERF & $7 / 6 / 00$ & $7 / 11 / 00$ & 366,700 & 0 & \\
\hline & & & WATER & AP-108 & $8 / 17 / 00$ & $8 / 17 / 00$ & 35,000 & 0 & \\
\hline & & & WCE88 & AP-108 & $8 / 17 / 00$ & $8 / 18 / 00$ & 141,000 & 0 & \\
\hline $\mathbf{Y}$ & $Y$ & $Y$ & AZ-10I & AY-101 & $8 / 18 / 00$ & $8 / 21 / 00$ & 685,000 & 0 & $\begin{array}{l}\text { Pre-stage } \\
\text { Envelope B/Initial } \\
\text { Decant Envelope } \\
\text { D }\end{array}$ \\
\hline & $Y$ & & $\begin{array}{l}\text { WASH- } \\
\text { WATER }\end{array}$ & $A Z-101$ & $8 / 21 / 00$ & $8 / 22 / 00$ & 146,000 & 0 & First Wash $1 \mathrm{AZ}$ \\
\hline & & & TNS88 & AP-106 & $9 / 17 / 00$ & $9 / 17 / 00$ & 1,500 & 500 & \\
\hline & & & WESF & AP-106 & $9 / 17 / 00$ & 9/17/00 & 10,000 & 0 & \\
\hline & & & PXTCO & AP-106 & $9 / 17 / 00$ & 9/17/00 & 10,000 & 0 & $\cdot$ \\
\hline & & & WATER & AP-106 & $9 / 17 / 00$ & $9 / 17 / 00$ & 40,000 & 0 & \\
\hline & & & TAL88 & AP-106 & $9 / 17 / 00$ & $9 / 17 / 00$ & 54,000 & 0 & \\
\hline & & & 1 NS96 & AW-105 & $9 / 18 / 00$ & $9 / 18 / 00$ & 15,002 & 4,998 & \\
\hline & & & $34 \mathrm{~L} 87$ & AP-106 & $9 / 17 / 00$ & $9 / 18 / 00$ & 137,000 & 0 & \\
\hline & & & AW-106 & AP-107 & $9 / 14 / 00$ & $9 / 18 / 00$ & 778,200 & 34 & \\
\hline & & & WATER & AW-105 & $9 / 18 / 00$ & $9 / 18 / 00$ & 174,000 & 0 & \\
\hline & & & WATER & AP-106 & $9 / 18 / 00$ & $9 / 19 / 00$ & 30,000 & 0 & \\
\hline & & & EVAPF & AP-106 & $9 / 17 / 00$ & $9 / 19 / 00$ & 320,000 & 0 & \\
\hline & & & 1FL96 & AW-105 & $9 / 18 / 00$ & $9 / 19 / 00$ & 53,000 & 0 & \\
\hline & & & $1 \mathrm{KL} 96$ & AW-105 & $9 / 18 / 00$ & 9/19/00 & 332,000 & 0 & \\
\hline $\mathbf{Y}$ & & $\mathrm{Y}$ & $\begin{array}{l}\text { WASH- } \\
\text { WATER }\end{array}$ & AP-104 & $10 / 1 / 00$ & $10 / 2 / 00$ & 200,000 & 0 & Flush 4AP \\
\hline$Y$ & & $\mathrm{X}$ & AP-106 & AW-102 & $10 / 2 / 00$ & $10 / 4 / 00$ & 474,000 & 16 & Empty 6AP \\
\hline
\end{tabular}


Table A-1. Projected Transfers. Through October 2011. (26 sheets)

Note: This table is extracted from Table H-1 of the Tank Waste Remediation System

Operations and Utilization Plan (Kirkbride et al. 1997)

\begin{tabular}{|c|c|c|c|c|c|c|c|c|c|}
\hline $\begin{array}{l}\text { Boot } \\
\text { Strap }\end{array}$ & $\begin{array}{c}\text { HLW } \\
\text { Flag }\end{array}$ & $\begin{array}{l}\text { LAW } \\
\text { Flag }\end{array}$ & From & To & $\begin{array}{l}\text { Start } \\
\text { Date }\end{array}$ & $\begin{array}{l}\text { End } \\
\text { Date }\end{array}$ & $\begin{array}{c}\text { Liquid } \\
\text { (gallons) }\end{array}$ & $\begin{array}{c}\text { Solid } \\
\text { (Gallons) }\end{array}$ & Notes \\
\hline $\mathrm{Y}$ & & $\mathrm{Y}$ & AP-106 & AW-102 & $10 / 5 / 00$ & $10 / 5 / 00$ & 71,998 & 2 & Empty 6AP \\
\hline $\mathrm{Y}$ & & $Y$ & AP-106 & AW-102 & $10 / 6 / 00$ & $10 / 6 / 00$ & 130,000 & 4 & Empty 6AP \\
\hline \multirow[t]{2}{*}{$\mathbf{Y}$} & & $\mathrm{Y}$ & AP-106 & AW-102 & $10 / 7 / 00$ & $10 / 7 / 00$ & 60,191 & 2 & Empty 6AP \\
\hline & & & AW-105 & AW-102 & $10 / 6 / 00$ & $10 / 8 / 00$ & 414,000 & 14 & \\
\hline \multirow[t]{14}{*}{$\mathrm{Y}$} & & $\mathbf{Y}$ & AP-106 & AW-102 & $10 / 8 / 00$ & $10 / 8 / 00$ & 0 & 0 & Empty $6 \mathrm{AP}$ \\
\hline & & . & AW-105 & $A W-102$ & $10 / 9 / 00$ & $10 / 10 / 00$ & 213,100 & 7 & $\cdot$ \\
\hline & $\mathbf{Y}$ & & AZ-101 & AW-105 & $10 / 10 / 00$ & $10 / 10 / 00$ & 142,500 & 0 & $\begin{array}{l}\text { First Wash } \\
\text { Decant IAZ }\end{array}$ \\
\hline & & & AW-102 & $242-A$ & $10 / 5 / 00$ & $10 / 12 / 00$ & $1,476,000$ & 52 & $\cdot$ \\
\hline & & & $242-\mathrm{A}$ & AW-106 & $10 / 5 / 00$ & $10 / 12 / 00$ & 87,361 & 52 & \\
\hline & & & $242-A$ & LERF & $10 / 5 / 00$ & $10 / 12 / 00$ & $1,389,000$ & 0 & \\
\hline & $\mathrm{Y}$ & & $\begin{array}{l}\text { WASH- } \\
\text { WATER }\end{array}$ & $A Z-101$ & $10 / 11 / 00$ & $10 / 12 / 00$ & 146,000 & 0 & $\begin{array}{l}\text { Second Wash } \\
1 \mathrm{AZ}\end{array}$ \\
\hline & & $\mathrm{Y}$ & AP-104 & AP-102 & $10 / 15 / 00$ & $10 / 16 / 00$ & 200,000 & 1 & Flush 2AP \\
\hline & & & AW-106 & AP-107 & $11 / 3 / 00$ & $11 / 3 / 00$ & 86,999 & 5 & \\
\hline & $\mathrm{Y}$ & & AZ-101 & $A W-105$ & $11 / 30 / 00$ & $12 / 1 / 00$ & 140,200 & 0 & $\begin{array}{l}\text { Second Wash } \\
\text { Decant 1AZ }\end{array}$ \\
\hline & $\mathrm{Y}$ & & $\begin{array}{l}\text { WASH- } \\
\text { WATER }\end{array}$ & $A Z-101$ & $12 / 2 / 00$ & $12 / 3 / 00$ & 146,000 & 0 & Third Wash IAZ \\
\hline & $\mathbf{Y}$ & & AZ-101 & AW-10S & $1 / 21 / 01$ & $1 / 21 / 01$ & 142,900 & 0 & $\begin{array}{l}\text { Third Wash } \\
\text { Decant } 1 \mathrm{AZ}\end{array}$ \\
\hline & $\mathbf{Y}$ & & $\begin{array}{l}\text { WASH- } \\
\text { WATER }\end{array}$ & $A Z-101$ & $1 / 22 / 01$ & $1 / 23 / 01$ & 140,200 & 0 & $\begin{array}{l}\text { Transfer Water } \\
\text { Add } 1 \mathrm{AZ}\end{array}$ \\
\hline & & & AP-106 & AW-102 & $2 / 7 / 01$ & $2 / 7 / 01$ & 0 & 0 & \\
\hline \multirow[t]{12}{*}{$\mathrm{Y}$} & & $Y$ & AP-102 & AP-106 & $2 / 15 / 01$ & $2 / 16 / 01$ & 200,000 & 1 & Flush 6AP \\
\hline & & & ZNL87 & SY-102 & $3 / 20 / 01$ & $3 / 20 / 01$ & 4,000 & 0 & \\
\hline & & & WCW88 & SY-102 & $3 / 20 / 01$ & $3 / 20 / 01$ & 45,000 & 0 & \\
\hline & & & SPN87 & SY-102 & $3 / 20 / 01$ & $3 / 20 / 01$ & 37,000 & 0 & \\
\hline & & & WATER & SY-102 & $3 / 20 / 01$ & $3 / 20 / 01$ & 37,000 & 0 & \\
\hline & & & WNW88 & SY-102 & $3 / 20 / 01$ & $3 / 20 / 01$ & 83,000 & 0 & \\
\hline & & $\mathbf{Y}$ & AN-105 & AP-102 & $3 / 17 / 01$ & $3 / 20 / 01$ & 542,400 & 7,895 & $\begin{array}{l}\text { Retrieve/Stage } \\
\text { LAW Batch 1, } \\
\text { Contractor 1, } \\
\text { Envelope A } \\
\end{array}$ \\
\hline & & $Y$ & LERF & \begin{tabular}{|l} 
AN-105- \\
PUMP
\end{tabular} & $3 / 17 / 01$ & $3 / 20 / 01$ & 154,100 & 0 & Dilution Water \\
\hline & & $\mathrm{Y}$ & AN-105 & $\mathrm{AP}-102$ & $3 / 17 / 01$ & $3 / 20 / 01$ & 686,500 & 294 & As-received Ghost \\
\hline & & $X$ & AN-105 & AP-104 & $3 / 21 / 01$ & $3 / 24 / 01$ & 542,400 & 7,895 & $\begin{array}{l}\text { Retrieve/Stage } \\
\text { LAW Batch 1, } \\
\text { Contractor 2, } \\
\text { Envelope A }\end{array}$ \\
\hline & & $\mathbf{Y}$ & LERF & $\begin{array}{l}\text { AN-105- } \\
\text { PUMP }\end{array}$ & $3 / 21 / 01$ & $3 / 24 / 01$ & 154,100 & 0 & Dilution Water \\
\hline & & $\mathbf{Y}$ & AN-105 & AP-104 & $3 / 21 / 01$ & $3 / 24 / 01$ & 686,500 & 294 & As-received Ghost \\
\hline
\end{tabular}


HNF-2168

Revision 0

Table A-1. Projected Transfers Through October 2011. (26 sheets)

Note: This table is extracted from Table $\mathrm{H}-1$ of the Tank Waste Remediation System

Operations and Utilization Plan (Kirkbride et al. 1997).

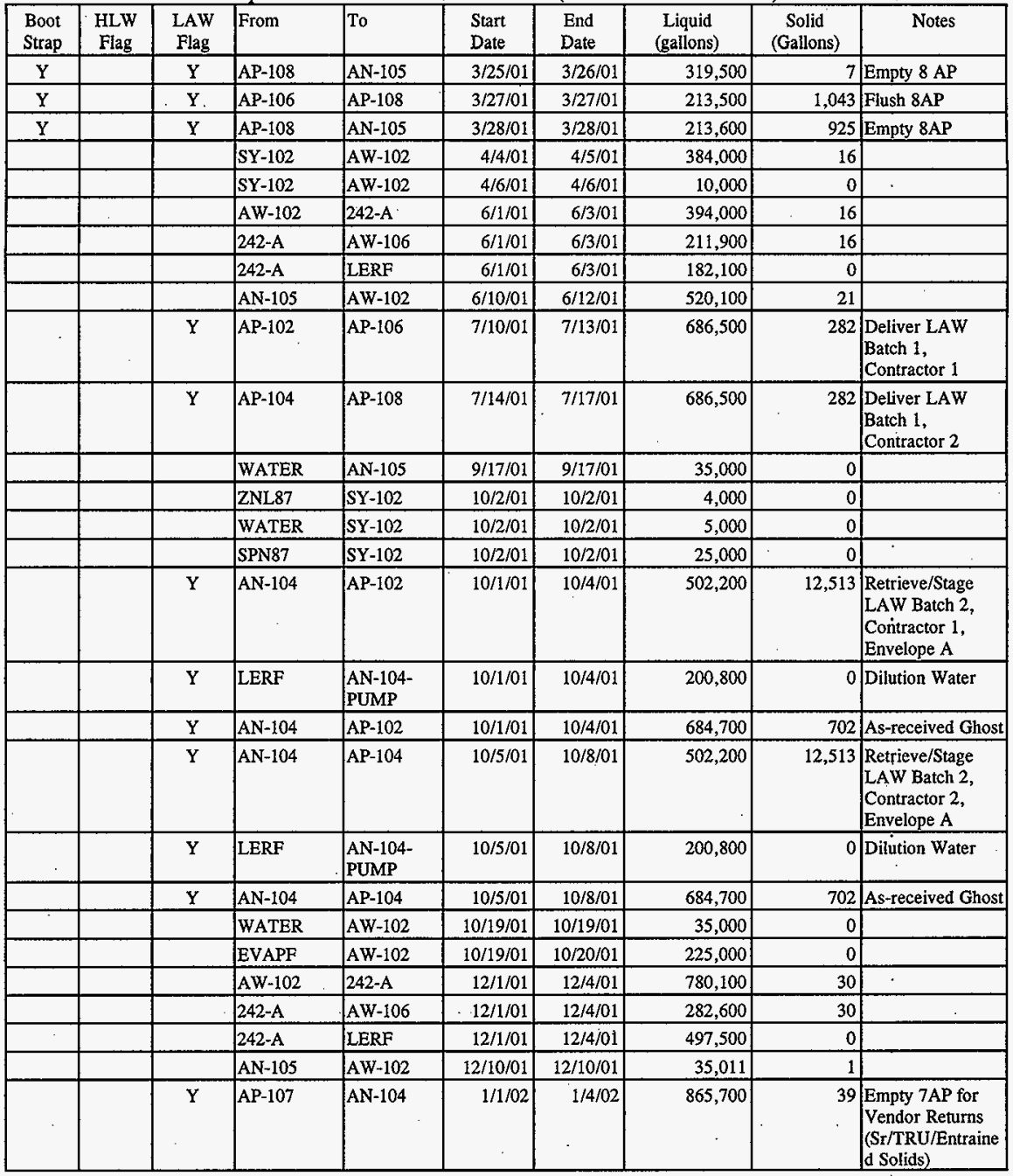


HNF-2168

Revision 0

Table A-1. Projected Transfers Through October 2011. (26 sheets)

Note: This table is extracted from Table H-1 of the Tank Waste Remediation System

Operations and Utilization Plan (Kirkbride et al. 1997)

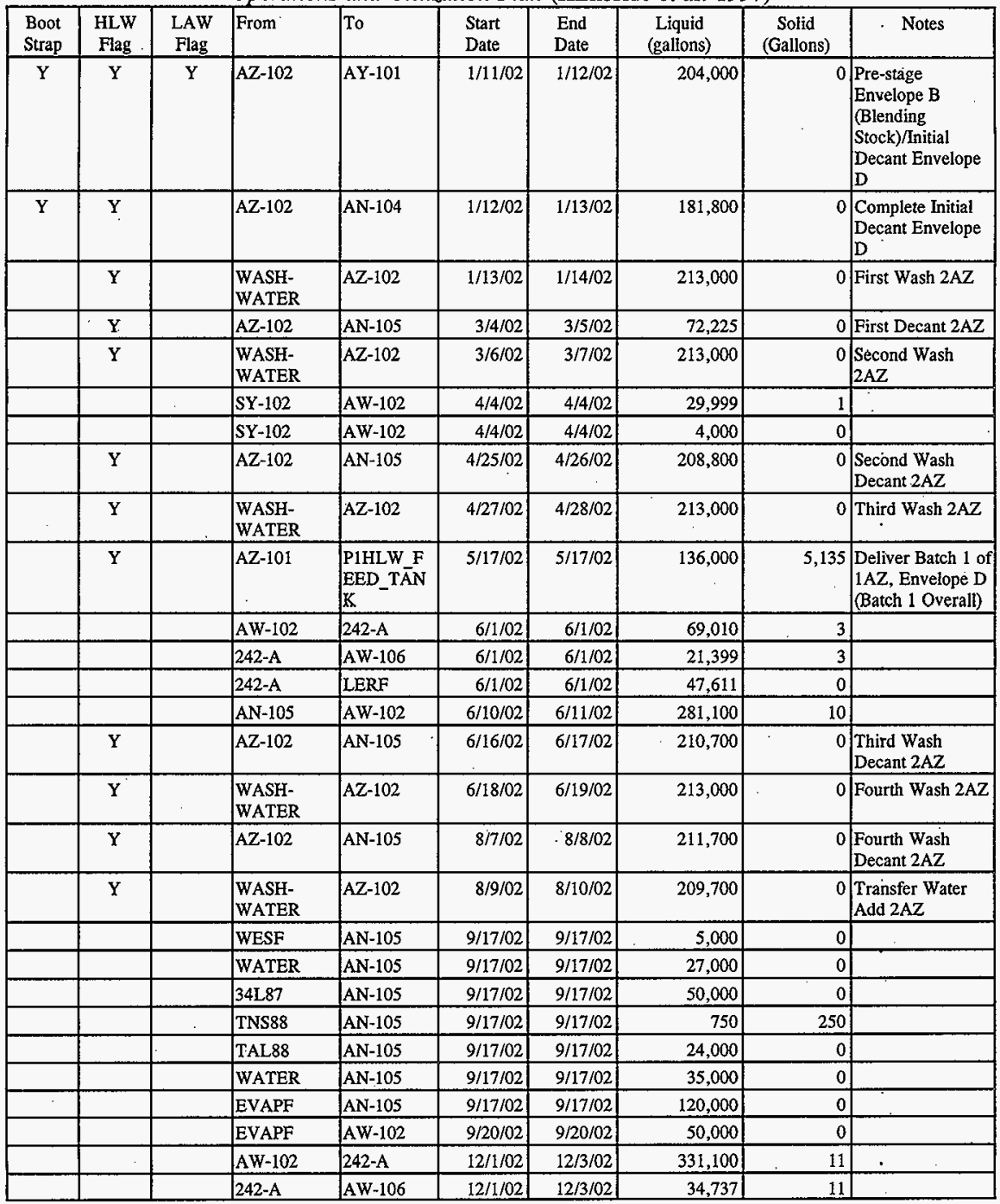


HNF-2168

Revision 0

Table A-1. Projected Transfers Through October 2011. (26 sheets)

Note: This table is extracted from Table H-1 of the Tank Waste Remediation System Operations and Utilization Plan (Kirkbride et al. 1997)

\begin{tabular}{|c|c|c|c|c|c|c|c|c|c|}
\hline $\begin{array}{l}\text { Boot } \\
\text { Strap }\end{array}$ & $\begin{array}{c}\text { HLW } \\
\text { Flag }\end{array}$ & $\begin{array}{l}\text { LAW } \\
\text { Flag }\end{array}$ & From & To & $\begin{array}{l}\text { Start } \\
\text { Date }\end{array}$ & $\begin{array}{l}\text { End } \\
\text { Date }\end{array}$ & $\begin{array}{c}\text { Liquid } \\
\text { (gallons) }\end{array}$ & $\begin{array}{c}\text { Solid } \\
\text { (Gallons) }\end{array}$ & Notes \\
\hline & & & 242-A & LERF & $12 / 1 / 02$ & $12 / 3 / 02$ & 296,400 & 0 & \\
\hline & & $\mathrm{Y}$ & RETURN_1 & AP-107 & $12 / 3 / 02$ & $12 / 3 / 02$ & 8,706 & 295 & \begin{tabular}{|l|} 
Sr/TRU \& \\
Entrained Solids \\
Return from LAW \\
Contractor 1 \\
\end{tabular} \\
\hline & & & AN-105 & AW-102 & $12 / 10 / 02$ & $12 / 13 / 02$ & 681,500 & 23 & \\
\hline & & $\mathrm{Y}$ & RETURN_2 & AP-107 & $1 / 2 / 03$ & $1 / 2 / 03$ & 8,669 & 331 & $\begin{array}{l}\text { Entrained Solids } \\
\text { Return from LAW } \\
\text { Contractor } 2\end{array}$ \\
\hline & & $\mathrm{Y}$ & AP-102 & AP-106 & $1 / 5 / 03$ & $1 / 9 / 03$ & 684,700 & 686 & $\begin{array}{l}\text { Deliver LAW } \\
\text { Batch 2, } \\
\text { Contractor 1 } \\
\end{array}$ \\
\hline & & $\mathrm{Y}$ & AP-104 & AP-108 & $1 / 9 / 03$ & $1 / 12 / 03$ & 684,700 & 686 & \begin{tabular}{|l|} 
Deliver LAW \\
Batch 2, \\
Contractor 2 \\
\end{tabular} \\
\hline & & $\mathbf{Y}$ & AW-101 & AP-102 & $1 / 9 / 03$ & $1 / 13 / 03$ & 537,200 & 13,013 & $\begin{array}{l}\text { Retrieve/Stage } \\
\text { LAW Batch } 3 \text {, } \\
\text { Contractor } 1 \text {, } \\
\text { Envelope A }\end{array}$ \\
\hline & & $\mathrm{Y}$ & LERF & \begin{tabular}{|l} 
AW-101- \\
PUMP \\
\end{tabular} & $1 / 9 / 03$ & $1 / 13 / 03$ & 308,100 & 0 & Dilution Water \\
\hline & & $Y$ & AW-101 & AP-102 & $1 / 9 / 03$ & $1 / 13 / 03$ & 845,400 & 13,013 & As-received Ghost \\
\hline & & $\mathrm{Y}$ & AW-101 & AP-104 & $1 / 14 / 03$ & $1 / 18 / 03$ & 537,200 & 13,013 & $\begin{array}{l}\text { Retrieve/Stage } \\
\text { LAW Batch 3, } \\
\text { Contractor 2, } \\
\text { Envelope A }\end{array}$ \\
\hline & & $\mathbf{Y}$ & LERF & $\begin{array}{l}\text { AW-101- } \\
\text { PUMP }\end{array}$ & $1 / 14 / 03$ & $1 / 18 / 03$ & 308,100 & 0 & Dilution Water \\
\hline & & $Y$ & AW-101 & AP-104 & $1 / 14 / 03$ & $1 / 18 / 03$ & 845,400 & 13,013 & As-received Ghost \\
\hline & $Y$ & & $\begin{array}{l}\text { PIHLW_FE } \\
\text { ED_TANKK }\end{array}$ & $\begin{array}{l}\text { HLW- } \\
\text { CONTRAC } \\
\text { TOR }\end{array}$ & $6 / 1 / 02$ & $2 / 24 / 03$ & 136,000 & 5,135 & $\begin{array}{l}\text { Process Batch 1 } \\
\text { of 1AZ, Envelope } \\
\text { D (Batch 1 } \\
\text { Overall) } \\
\end{array}$ \\
\hline & $\mathrm{Y}$ & & AZ-10I & $\begin{array}{l}\text { P1HLW_F } \\
\text { EED_TANN } \\
\text { K }\end{array}$ & $2 / 24 / 03$ & $2 / 25 / 03$ & 136,000 & 5,135 & $\begin{array}{l}\text { Deliver Batch } 2 \text { of } \\
\text { 1AZ, Envelope D } \\
\text { (Batch } 2 \text { Overall) } \\
\end{array}$ \\
\hline & & & AW-105 & AW-101 & $3 / 1 / 03$ & $3 / 3 / 03$ & 550,400 & 26 & \\
\hline & & & ZNL87 & SY-102 & $3 / 20 / 03$ & $3 / 20 / 03$ & 3,000 & 0 & \\
\hline & & & WATER & SY-102 & $3 / 20 / 03$ & $3 / 20 / 03$ & 6,000 & 0 & \\
\hline & & & SPN87 & SY-102 & $3 / 20 / 03$ & $3 / 20 / 03$ & 25,000 & 0 & \\
\hline & & & SY-102 & AW-102 & $4 / 4 / 03$ & $4 / 4 / 03$ & 29,999 & 1 & \\
\hline & & & SY-102 & AW-102 & $4 / 4 / 03$ & $4 / 4 / 03$ & 4,000 & 0 & \\
\hline & & $Y$ & RETURN_1 & AP-107 & $4 / 28 / 03$ & $4 / 28 / 03$ & 8,679 & 325 & $\begin{array}{l}\text { Sr/TRU \& } \\
\text { Entrained Solids } \\
\text { Return from LAW } \\
\text { Contractor 1 }\end{array}$ \\
\hline
\end{tabular}


Table A-1. Projected Transfers Through October 2011. (26 sheets)

Note: This table is extracted from Table $\mathrm{H}-1$ of the Tank Waste Remediation System

Operations and Utilization Plan (Kirkbride et al. 1997)

\begin{tabular}{|c|c|c|c|c|c|c|c|c|c|}
\hline $\begin{array}{l}\text { Boot } \\
\text { Strap } \\
\end{array}$ & $\begin{array}{c}\text { HLW } \\
\text { Flag }\end{array}$ & $\begin{array}{c}\text { LAW } \\
\text { Flag }\end{array}$ & From & To & $\begin{array}{l}\text { Start } \\
\text { Date }\end{array}$ & $\begin{array}{l}\text { End } \\
\text { Date }\end{array}$ & $\begin{array}{c}\text { Liquid } \\
\text { (gallons) }\end{array}$ & $\begin{array}{c}\text { Solid } \\
\text { (Gallons) } \\
\end{array}$ & Notes \\
\hline & & $\mathbf{Y}$ & RETURN_2 & AP-107 & $5 / 22 / 03$ & $5 / 23 / 03$ & 8,672 & 332 & \begin{tabular}{|l|} 
Entrained Solids \\
Return from LAW \\
Contractor 2 \\
\end{tabular} \\
\hline & & & AW-102 & $242-A$ & $6 / 1 / 03$ & $6 / 4 / 03$ & 715,500 & 24 & \\
\hline - & & & $242-\mathrm{A}$ & AW-106 & $6 / 1 / 03$ & $6 / 4 / 03$ & 34,944 & 24 & \\
\hline & & & $242-A$ & LERF & $6 / 1 / 03$ & $6 / 4 / 03$ & 680,600 & 0 & \\
\hline & & & AN-105 & $A W-102$ & $6 / 10 / 03$ & $6 / 10 / 03$ & 0 & 0 & \\
\hline & & & AW-106 & AW-101 & $7 / 1 / 03$ & $7 / 1 / 03$ & 34,750 & 2 & \\
\hline & & $Y$ & RETURN_1 & AP-107 & $9 / 12 / 03$ & $9 / 12 / 03$ & 8,674 & 332 & \begin{tabular}{|l|} 
Sr/TRU \& \\
Entrained Solids \\
Return from LAW \\
Contractor 1 \\
\end{tabular} \\
\hline & & & TNS88 & $A N-105$ & 9/17/03 & $9 / 17 / 03$ & 750 & 250 & \\
\hline & & & WESF & AN-105 & $9 / 17 / 03$ & $9 / 17 / 03$ & 5,000 & 0 & \\
\hline & & & TAL88 & AN-105 & $9 / 17 / 03$ & $9 / 17 / 03$ & 24,000 & 0 & \\
\hline & & & WATER & AN-105 & 9/17/03 & $9 / 17 / 03$ & 35,000 & 0 & \\
\hline & & & $34 \mathrm{~L} 87$ & AN-105 & $9 / 17 / 03$ & $9 / 17 / 03$ & 57,000 & 0 & \\
\hline & & & WATER & AN-105 & $9 / 17 / 03$ & $9 / 17 / 03$ & 8,000 & 0 & \\
\hline & & & EVAPF & AN-105 & $9 / 17 / 03$ & $9 / 17 / 03$ & 120,000 & 0 & \\
\hline & & & WATER & AN-105 & $9 / 18 / 03$ & $9 / 18 / 03$ & 22,000 & 0 & $:$ \\
\hline & & & EVAPF & AW-102 & $9 / 20 / 03$ & $9 / 20 / 03$ & 35,000 & 0 & \\
\hline & & $\mathbf{Y}$ & AP-102 & AP-106 & $9 / 29 / 03$ & $10 / 2 / 03$ & 740,300 & 1,581 & $\begin{array}{l}\text { Deliver LAW } \\
\text { Batch 3, } \\
\text { Contractor 1 } \\
\end{array}$ \\
\hline & & Y & RETURN_2 & AP-107 & $10 / 6 / 03$ & $10 / 6 / 03$ & 8,669 & 332 & $\begin{array}{l}\text { Entrained Solids } \\
\text { Return from LAW } \\
\text { Contractor } 2 \\
\end{array}$ \\
\hline & & $\mathbf{Y}$ & AP-104 & AP-108 & $10 / 2 / 03$ & $10 / 6 / 03$ & 740,300 & 1,581 & $\begin{array}{l}\text { Deliver LAW } \\
\text { Batch 3, } \\
\text { Contractor } 2 \\
\end{array}$ \\
\hline & & $\mathbf{Y}$ & AN-103 & AP-102 & $10 / 3 / 03$ & $10 / 6 / 03$ & 436,600 & 28,133 & $\begin{array}{l}\text { Retrieve/Stage } \\
\text { LAW Batch } 4 \text {, } \\
\text { Contractor } 1 \text {, } \\
\text { Envelope A }\end{array}$ \\
\hline & & $\mathrm{Y}$ & LERF & $\begin{array}{l}\text { AN-103- } \\
\text { PUMP }\end{array}$ & $10 / 3 / 03$ & $10 / 6 / 03$ & 264,900 & 0 & Dilution Water \\
\hline & & $\mathrm{Y}$ & AN-103 & AP-102 & $10 / 3 / 03$ & $10 / 6 / 03$ & 738,300 & 549 & As-received Ghost \\
\hline & & $\mathbf{Y}$ & AN-103 & AP-104 & $10 / 7 / 03$ & $10 / 10 / 03$ & 436,600 & 28,133 & $\begin{array}{l}\text { Retrieve/Stage } \\
\text { LAW Batch 4, } \\
\text { Contractor 2, } \\
\text { Envelope A } \\
\end{array}$ \\
\hline & & $\mathrm{Y}$ & LERF & $\begin{array}{l}\text { AN-103- } \\
\text { PUMP }\end{array}$ & $10 / 7 / 03$ & $10 / 10 / 03$ & 264,900 & 0 & Dilution Water \\
\hline & & $\mathbf{Y}$ & AN-103 & AP-104 & $10 / 7 / 03$ & $10 / 10 / 03$ & 738,300 & 549 & As-received Ghost \\
\hline
\end{tabular}




\section{Revision 0}

Table A-1. Projected Transfers Through October 2011. (26 sheets)

Note: This table is extracted from Table H-1 of the Tank Waste Remediation System

Operations and Utilization Plan (Kirkbride et al. 1997)

\begin{tabular}{|c|c|c|c|c|c|c|c|c|c|}
\hline $\begin{array}{l}\text { Boot } \\
\text { Strap }\end{array}$ & $\begin{array}{l}\text { HLW } \\
\text { Flag }\end{array}$ & $\begin{array}{c}\text { LAW } \\
\text { Flag }\end{array}$ & From & To & $\begin{array}{l}\text { Start } \\
\text { Date }\end{array}$ & $\begin{array}{l}\text { End } \\
\text { Date }\end{array}$ & $\begin{array}{c}\text { Liquid } \\
\text { (gallons) }\end{array}$ & $\begin{array}{c}\text { Solid } \\
\text { (Gallons) }\end{array}$ & - Notes \\
\hline & & $\mathrm{Y}$ & RETURN_1 & AP-107 & $11 / 14 / 03$ & $11 / 14 / 03$ & 8,675 & 330 & $\begin{array}{l}\text { Sr/TRU \& } \\
\text { Entrained Solids } \\
\text { Return from LAW } \\
\text { Contractor } 1 \\
\end{array}$ \\
\hline & $\mathbf{Y}$ & & $\begin{array}{l}\text { P1HLW_FE } \\
\text { ED_TAÑK }\end{array}$ & \begin{tabular}{|l} 
HLW- \\
CONTRAC \\
TOR
\end{tabular} & $2 / 25 / 03$ & $11 / 20 / 03$ & 136,000 & 5,135 & $\begin{array}{l}\text { Process Batch } 2 \\
\text { of 1AZ, Envelope } \\
\text { D (Batch } 2 \\
\text { Overall) } \\
\end{array}$ \\
\hline & $Y$ & & AZ-102 & $\begin{array}{l}\text { P1HLW_F } \\
\text { EED_TÂN } \\
\text { K }\end{array}$ & $11 / 20 / 03$ & $11 / 21 / 03$ & 145,900 & 6,032 & $\begin{array}{l}\text { Deliver Batch } 1 \text { of } \\
\text { 2AZ, Envelope D } \\
\text { (Batch } 3 \text { Overall) }\end{array}$ \\
\hline & & $\mathbf{Y}$ & RETURN_2 & AP-107 & $11 / 25 / 03$ & $11 / 25 / 03$ & 8,675 & 330 & \begin{tabular}{|l|} 
Entrained Solids \\
Return from LAW \\
Contractor 2 \\
\end{tabular} \\
\hline & & & AW-102 & $242-\mathrm{A}$ & $12 / 1 / 03$ & $12 / 1 / 03$ & 35,011 & 1 & \\
\hline & & & $242-\mathrm{A}$ & AW-106 & $12 / 1 / 03$ & $12 / 1 / 03$ & 756 & 1. & \\
\hline & & & $242-\mathrm{A}$ & LERF & $12 / 1 / 03$ & $12 / 1 / 03$ & 34,256 & 0 & . \\
\hline & & & AN-105 & AW-102 & $12 / 10 / 03$ & $12 / 11 / 03$ & 268,900 & 9 & \\
\hline & & & AW-106 & AW-101 & $1 / 1 / 04$ & $1 / 1 / 04$ & 746 & 0 & \\
\hline & & $Y$ & RETURN_1 & AP-107 & $1 / 3 / 04$ & $1 / 3 / 04$ & 8,674 & $\begin{array}{l}330 \\
\end{array}$ & $\begin{array}{l}\text { Sr/TRU \& } \\
\text { Entrained Solids } \\
\text { Return from LAW } \\
\text { Contractor } \\
\end{array}$ \\
\hline & & $\mathrm{Y}$ & RETURN_2 & AP-107 & $1 / 14 / 04$ & $1 / 14 / 04$ & 8,674 & 330 & $\begin{array}{l}\text { Entrained Solids } \\
\text { Return from LAW } \\
\text { Contractor } 2 \\
\end{array}$ \\
\hline & & $\bar{Y}$ & RETURN_1 & AP-107 & $2 / 22 / 04$ & $2 / 22 / 04$ & 8,674 & 330 & $\begin{array}{l}\text { Sr/TRU \& } \\
\text { Entrained Solids } \\
\text { Return from LAW } \\
\text { Contractor 1 } \\
\end{array}$ \\
\hline & & $\mathbf{Y}$ & RETURN_2 & AP-107 & $3 / 3 / 04$ & $3 / 3 / 04$ & 8,674 & 330 & $\begin{array}{l}\text { Entrained Solids } \\
\text { Return from LAW } \\
\text { Contractor } 2 \\
\end{array}$ \\
\hline & & & WATER & SY-102 & $3 / 20 / 04$ & $3 / 20 / 04$ & 5,000 & 0 & \\
\hline & & & ZNL87 & SY-102 & $3 / 20 / 04$ & $3 / 20 / 04$ & 3,000 & 0 & $\cdot$ \\
\hline & & & SPN87. & $S Y-102$ & $3 / 20 / 04$ & $3 / 20 / 04$ & 25,000 & 0 & \\
\hline & & & SY-102 & AW-102 & $4 / 4 / 04$ & $4 / 4 / 04$ & 29,999 & 1 & \\
\hline & & & $S Y-102$ & AW-102 & $4 / 4 / 04$ & $4 / 4 / 04$ & 3,000 & 0 & \\
\hline & & $\mathrm{Y}$ & RETURN_1 & AP-107 & $4 / 13 / 04$ & $4 / 13 / 04$ & 8,674 & 330 & $\begin{array}{l}\text { Sr/TRU \& } \\
\text { Entrained Solids } \\
\text { Return from LAW } \\
\text { Contractor 1 } \\
\end{array}$ \\
\hline & & $\bar{Y}$ & RETURN_2 & AP-107 & $4 / 22 / 04$ & $4 / 22 / 04$ & 8,674 & 330 & $\begin{array}{l}\text { Entrained Solids } \\
\text { Return from LAW } \\
\text { Contractor } 2 \\
\end{array}$ \\
\hline & & $\mathrm{Y}$ & AP-102 & AP-106 & $5 / 3 / 04$ & $5 / 7 / 04$ & 753,200 & 1,687 & $\begin{array}{l}\text { Deliver LAW } \\
\text { Batch 4, } \\
\text { Contractor } 1 \\
\end{array}$ \\
\hline
\end{tabular}


HNF-2168

Revision 0

Table A-1. Projected Transfers Through October 2011. (26 sheets)

Note: This table is extracted from Table $\mathrm{H}-1$ of the Tank Waste Remediation System

Operations and Utilization Plan (Kirkbride et al. 1997)

\begin{tabular}{|c|c|c|c|c|c|c|c|c|c|}
\hline $\begin{array}{l}\text { Boot } \\
\text { Strap }\end{array}$ & $\begin{array}{l}\text { HLW } \\
\text { Flag }\end{array}$ & $\begin{array}{l}\text { LAW } \\
\text { Flag }\end{array}$ & From & To & $\begin{array}{l}\text { Start } \\
\text { Date }\end{array}$ & $\begin{array}{l}\text { End } \\
\text { Date }\end{array}$ & $\begin{array}{c}\text { Liquid } \\
\text { (gallons) }\end{array}$ & $\begin{array}{c}\text { Solid } \\
\text { (Gallons) }\end{array}$ & Notes \\
\hline & & $\bar{Y}$ & $\begin{array}{l}\text { WASH- } \\
\text { WATER }\end{array}$ & AP-102 & $5 / 8 / 04$ & $5 / 8 / 04$ & 118,100 & 0 & $\begin{array}{l}\text { Flush Water for } \\
\text { Solids Cleanout }\end{array}$ \\
\hline & & $Y$ & AP-104 & AP-108 & $5 / 7 / 04$ & $5 / 10 / 04$ & 753,200 & 1,687 & $\begin{array}{l}\text { Deliver LAW } \\
\text { Batch 4, } \\
\text { Contractor 2 } \\
\end{array}$ \\
\hline & & $\mathbf{Y}$ & $\begin{array}{l}\text { WASH- } \\
\text { WATER }\end{array}$ & AP-104 & $5 / 11 / 04$ & $5 / 11 / 04$ & 118,100 & 0 & $\begin{array}{l}\text { Fiush Water for } \\
\text { Solids Cleanout }\end{array}$ \\
\hline & & & AN-105 & AW-102 & $5 / 12 / 04$ & $5 / 12 / 04$ & 0 & 0 & \\
\hline & & $\mathrm{Y}$ & AP-102 & AN-105 & $5 / 13 / 04$ & $5 / 13 / 04$ & 209,500 & 9,168 & \begin{tabular}{|l} 
Cleanout \\
Accumulated \\
Solids
\end{tabular} \\
\hline & & $Y$ & AP-104 & AN-105 & $5 / 14 / 04$ & $5 / 14 / 04$ & 209,500 & 9,168 & \begin{tabular}{|l} 
Cleanout \\
Accumulated \\
Solids \\
\end{tabular} \\
\hline & & $\mathrm{Y}$ & $A X-101$ & AP-102 & $5 / 15 / 04$ & $5 / 16 / 04$ & 283,600 & 12 & $\begin{array}{l}\text { Stage LAW Batch } \\
5 \text {, Envelope B, } \\
\text { Contractor } 1 \\
\end{array}$ \\
\hline & & $\mathrm{Y}$ & AY-101 & AP-104 & $5 / 17 / 04$ & $5 / 18 / 04$ & 279,700 & 11 & $\begin{array}{l}\text { Stage LAW Batch } \\
5 \text {, Envelope B, } \\
\text { Contractor } 2 \\
\end{array}$ \\
\hline & & & AX-103 & AN-103 & $1 / 11 / 04$ & $5 / 29 / 04$ & 312,500 & 2,846 & \\
\hline & & & AW-102 & 242-A & $6 / 1 / 04$ & $6 / 2 / 04$ & 301,900 & 10 & \\
\hline & & & 242-A & AW-106 & $6 / 1 / 04$ & $6 / 2 / 04$ & 11,234 & 10 & \\
\hline & & & $242-\mathrm{A}$ & LERF & $6 / 1 / 04$ & $6 / 2 / 04$ & 290,700 & 0 & \\
\hline & & $\mathrm{Y}$ & RETURN_I & AP-107 & $6 / 5 / 04$ & $6 / 5 / 04$ & 8,675 & 331 & \begin{tabular}{|l} 
Sr/TRU \& \\
Entrained Solids \\
Return from LAW \\
Contractor 1 \\
\end{tabular} \\
\hline & & & AN-105 & AW-102 & $6 / 10 / 04$ & $6 / 11 / 04$ & 231,200 & 9 & \\
\hline & & $\dot{Y}$ & RETURN_2 & AP-107 & $6 / 15 / 04$ & $6 / 16 / 04$ & 8,682 & 331 & $\begin{array}{l}\text { Entrained Solids } \\
\text { Return from LAW } \\
\text { Contractor } 2\end{array}$ \\
\hline & & & AW-106 & AW-101 & $7 / 1 / 04$ & $7 / 1 / 04$ & 11,151 & 1 & \\
\hline & & $Y$ & RETURN_1 & AP-107 & $8 / 1 / 04$ & $8 / 1 / 04$ & 8,681 & 331 & \begin{tabular}{|l} 
Sr/TRU \& \\
Entrained Solids \\
Return from LAW \\
Contractor 1 \\
\end{tabular} \\
\hline & & $\mathbf{Y}$ & RETURN_2 & AP-107 & $8 / 11 / 04$ & $8 / 11 / 04$ & 8,678 & 331 & $\begin{array}{l}\text { Entrained Solids } \\
\text { Return from LAW } \\
\text { Contractor } 2 \\
\end{array}$ \\
\hline & & & C-103 & AN-103 & $5 / 29 / 04$ & $8 / 12 / 04$ & 290,500 & 10,424 & \\
\hline & & & TNS88 & AN-105 & $9 / 17 / 04$ & $9 / 17 / 04$ & 750 & 250 & \\
\hline & & & ZNL87 & SY-102 & $9 / 17 / 04$ & $9 / 17 / 04$ & 3,000 & 0 & \\
\hline & & & SPN87 & SY-102 & $9 / 17 / 04$ & $9 / 17 / 04$ & 25,000 & 0 & \\
\hline & & & TAL88 & AN-105 & $9 / 17 / 04$ & 9/17/04 & 24,000 & 0 & \\
\hline & & & $34 L 87$ & AN-105 & $9 / 17 / 04$ & $9 / 17 / 04$ & 50,000 & 0 & \\
\hline
\end{tabular}


Table A-1. Projected Transfers Through October 2011. (26 sheets)

Note: This table is extracted from Table H-1 of the Tank Waste Remediation System Operations and Utilization Plan (Kirkbride et al. 1997)

\begin{tabular}{|c|c|c|c|c|c|c|c|c|c|}
\hline $\begin{array}{l}\text { Boot } \\
\text { Strap } \\
\end{array}$ & $\begin{array}{c}\text { HLW } \\
\text { Flag } \\
\end{array}$ & $\begin{array}{l}\text { LAW } \\
\text { Flag } \\
\end{array}$ & From & To & $\begin{array}{l}\text { Start } \\
\text { Date }\end{array}$ & $\begin{array}{l}\text { End } \\
\text { Date }\end{array}$ & $\begin{array}{c}\text { Liquid } \\
\text { (gallons) }\end{array}$ & $\begin{array}{c}\text { Solid } \\
\text { (Gallons) }\end{array}$ & Notes \\
\hline & & & WATER & AN-105 & $9 / 17 / 04$ & $9 / 17 / 04$ & 35,000 & 0 & \\
\hline & & & WESF & AN-105 & $9 / 17 / 04$ & $9 / 17 / 04$ & 5,000 & 0 & \\
\hline & & & EVAPF & AN-105 & $9 / 17 / 04$ & $9 / 17 / 04$ & 120,000 & 0 & \\
\hline & & & WATER & SY-102 & 9/1.7/04 & $9 / 19 / 04$ & 426,000 & 0 & \\
\hline & & & WATER & AN-10S & 9/19/04 & 9/19/04 & 27,000 & 0 & \\
\hline & & & WATER & SY-102 & 9/19/04 & 9/19/04 & 6,000 & 0 & \\
\hline & & & WATER & AW-105 & 9/19/04 & $9 / 20 / 04$ & 88,000 & 0 & \\
\hline & & & EVAPF & AW-102 & $9 / 20 / 04$ & $9 / 20 / 04$ & 85,000 & 0 & \\
\hline & & & 1FL96 & AW-105 & $9 / 19 / 04$ & $9 / 20 / 04$ & 200,000 & 0 & \\
\hline & $\mathbf{Y}$ & & $\begin{array}{l}\text { P1HLW FE } \\
\text { ED_TANKK }\end{array}$ & $\begin{array}{l}\text { HLW- } \\
\text { CONTRAC } \\
\text { TOR }\end{array}$ & $11 / 21 / 03$ & $9 / 26 / 04$ & 145,900 & 6,032 & $\begin{array}{l}\text { Process Batch } 1 \\
\text { of } 2 \mathrm{AZ} \text {, Envelope } \\
\mathrm{D} \text { (Batch } 3 \\
\text { Overall) } \\
\end{array}$ \\
\hline & & $Y$ & RETURN_1 & AP-107 & $9 / 27 / 04$ & $9 / 27 / 04$ & 8,681 & 331 & $\begin{array}{l}\text { Sr/TRU \& } \\
\text { Entrained Solids } \\
\text { Return from LAW } \\
\text { Contractor 1 } \\
\end{array}$ \\
\hline & $\mathrm{Y}$ & & AZ-102 & $\begin{array}{l}\text { P1HLW_F } \\
\text { EED_TAN } \\
\text { K }\end{array}$ & $9 / 26 / 04$ & $9 / 27 / 04$ & 145,900 & 6,032 & $\begin{array}{l}\text { Deliver Batch } 2 \text { of } \\
2 \mathrm{AZ}, \text { Envelope D } \\
\text { (Batch } 4 \text { Overali) } \\
\end{array}$ \\
\hline & & $\mathbf{Y}$ & RETURN_2 & AP-107 & $10 / 7 / 04$ & $10 / 7 / 04$ & 8,678 & 331 & $\begin{array}{l}\text { Entrained Solids } \\
\text { Return from LAW } \\
\text { Contractor } 2 \\
\end{array}$ \\
\hline & & $Y$ & RETURN_I & AP-107 & $11 / 23 / 04$ & $11 / 23 / 04$ & 8,681 & 331 & $\begin{array}{l}\text { Sr/TRU \& } \\
\text { Entrained Solids } \\
\text { Return from LAW } \\
\text { Contractor 1 } \\
\end{array}$ \\
\hline & & & A-102 & AN-103 & $8 / 12 / 04$ & $11 / 30 / 04$ & 102,200 & 744 & \\
\hline & & & AW-102 & $242-A$ & $12 / 1 / 04$ & $12 / 2 / 04$ & 316,200 & 12 & \\
\hline & & & $242-\mathrm{A}$ & AW-106 & $12 / 1 / 04$ & $12 / 2 / 04$ & 95,369 & 12 & \\
\hline & & & $242-\mathrm{A}$ & LERF & $12 / 1 / 04$ & $12 / 2 / 04$ & 220,900 & 0 & \\
\hline & & $Y$ & RETURN_2 & AP-107 & $12 / 3 / 04$ & $12 / 3 / 04$ & 8,678 & 331 & $\begin{array}{l}\text { Entrained Solids } \\
\text { Return from LAW } \\
\text { Contractor } 2 \\
\end{array}$ \\
\hline & & & AN-105 & AW-102 & $12 / 10 / 04$ & $12 / 11 / 04$ & 258,900 & 9 & \\
\hline & & & AW-106 & AW-101 & $1 / 1 / 05$ & $1 / 1 / 05$ & 95,306 & 4 & \\
\hline & & & AN-103 & AN-104 & $1 / 17 / 05$ & $1 / 17 / 05$ & 67,122 & 1,770 & \\
\hline & & & $\mathrm{AN}-103$ & AW-104 & $1 / 17 / 05$ & $1 / 18 / 05$ & 17,750 & 468 & \\
\hline & & & AN-103 & AW-103 & $1 / 18 / 05$ & $1 / 18 / 05$ & 3,897 & 103 & \\
\hline & & & $A N-103$ & AP-105 & $1 / 18 / 05$ & $1 / 18 / 05$ & 43,576 & 1,147 & \\
\hline & & & AN-103 & AP-103 & $1 / 18 / 05$ & $1 / 18 / 05$ & 42,878 & 1,128 & \\
\hline & & & AN-103 & AP-101 & $1 / 18 / 05$ & $1 / 18 / 05$ & 38,554 & 1,014 & $\cdot$ \\
\hline & & & AN-103 & $A N-101$ & $1 / 18 / 05$ & $1 / 19 / 05$ & 28,962 & 761 & \\
\hline
\end{tabular}


Table A-1. Projected Transfers Through October 2011. (26 sheets)

Note: This table is extracted from Table H-1 of the Tank Waste Remediation System

Operations and Utilization Plan (Kirkbride et al. 1997)

\begin{tabular}{|c|c|c|c|c|c|c|c|c|c|}
\hline $\begin{array}{l}\text { Boot } \\
\text { Strap }\end{array}$ & $\begin{array}{c}\text { HLW } \\
\text { Flag }\end{array}$ & $\begin{array}{c}\text { LAW } \\
\text { Flag }\end{array}$ & From & To & $\begin{array}{l}\text { Start } \\
\text { Date }\end{array}$ & $\begin{array}{l}\text { End } \\
\text { Date }\end{array}$ & $\begin{array}{c}\text { Liquid } \\
\text { (gallons) }\end{array}$ & $\begin{array}{c}\text { Solid } \\
\text { (Gallons) }\end{array}$ & Notes \\
\hline & . & $\mathbf{Y}$ & RETURN_1 & AP-107 & $1 / 19 / 05$ & $1 / 19 / 05$ & 8,681 & 331 & \begin{tabular}{|l|} 
Sr/TRU \& \\
Entrained Solids \\
Return from LAW \\
Contractor 1 \\
\end{tabular} \\
\hline & & $Y$ & RETURN_2 & AP-107 & $1 / 29 / 05$ & $1 / 29 / 05$ & 8,678 & 331 & $\begin{array}{l}\text { Entrained Solids } \\
\text { Return from LAW } \\
\text { Contractor } 2 \\
\end{array}$ \\
\hline & & $Y$ & RETURN_1 & AP-107 & $3 / 17 / 05$ & $3 / 17 / 05$ & 8,681 & 331 & \begin{tabular}{|l|} 
St/TRU \& \\
Entrained Solids \\
Return from LAW \\
Contractor 1 \\
\end{tabular} \\
\hline & & $\bar{Y}$ & AP-106 & \begin{tabular}{|l|} 
LAW- \\
CONTRAC \\
TOR-1
\end{tabular} & $6 / 1 / 02$ & $3 / 18 / 05$ & $2,865,000$ & 4,311 & $\begin{array}{l}\text { Process Envelope } \\
\text { A Batches } \\
\text { Contractor } 1 \\
\end{array}$ \\
\hline & & $\mathrm{Y}$ & AP-108 & \begin{tabular}{|l|} 
LAW- \\
CONTRAC \\
TOR-2
\end{tabular} & $6 / 1 / 02$ & $3 / 19 / 05$ & $2,865,000$ & 4,295 & $\begin{array}{l}\text { Process Envelope } \\
\text { A Batches } \\
\text { Contractor } 2 \\
\end{array}$ \\
\hline & & Y & AP-102 & AP-106 & $3 / 18 / 05$ & $3 / 20 / 05$ & 277,000 & 552 & $\begin{array}{l}\text { Deliver LAW } \\
\text { Batch 5, } \\
\text { Contractor 1, } \\
\text { Envelope B } \\
\end{array}$ \\
\hline & & $\mathbf{Y}$ & AP-104 & AP-108 & $3 / 20 / 05$ & $3 / 21 / 05$ & 273,000 & 544 & $\begin{array}{l}\text { Deliver LAW } \\
\text { Batch 5, } \\
\text { Contractor 2, } \\
\text { Envelope B } \\
\end{array}$ \\
\hline & & $Y$ & AN-107 & AP-102 & $3 / 21 / 05$ & $3 / 23 / 05$ & 481,700 & 22 & $\begin{array}{l}\text { Retrieve/Stage } \\
\text { LAW Batch } 6 / 7 \text {, } \\
\text { Contractor } 1 \text {, } \\
\text { Envelope C }\end{array}$ \\
\hline & & $Y$ & LERF & $\begin{array}{l}\text { AN-107- } \\
\text { PUMP }\end{array}$ & $3 / 21 / 05$ & $3 / 23 / 05$ & 72,259 & 0 & Dilution Water \\
\hline & & $\mathbf{Y}$ & AN-107 & AP-102 & $3 / 21 / 05$ & $3 / 23 / 05$ & 554,000 & 22 & As-received Ghost \\
\hline & & $\mathrm{Y}$ & AN-107 & AP-104 & $3 / 24 / 05$ & $3 / 26 / 05$ & 481,700 & 22 & $\begin{array}{l}\text { Retrieve/Stage } \\
\text { LAW Batch } 6 / 7, \\
\text { Contractor } 2, \\
\text { Envelope C } \\
\end{array}$ \\
\hline & & $\mathbf{Y}$ & LERF & $\begin{array}{l}\text { AN-107- } \\
\text { PUMP }\end{array}$ & $3 / 24 / 05$ & $3 / 26 / 05$ & 72,260 & 0 & Dilution Water \\
\hline & & $\mathrm{Y}$ & AN-107 & $\mathrm{AP}-104$ & $3 / 24 / 05$ & $3 / 26 / 05$ & 554,000 & 22 & As-received Ghost \\
\hline & & $\mathbf{Y}$ & RETURN_2 & AP-107 & $3 / 27 / 05$ & $3 / 27 / 05$ & 8,688 & 331 & $\begin{array}{l}\text { Entrained Solids } \\
\text { Return from LAW } \\
\text { Contractor } 2 \\
\end{array}$ \\
\hline $\mathrm{Y}$ & $\mathbf{Y}$ & & AY-102 & AN-105 & $3 / 26 / 05$ & $3 / 30 / 05$ & 724,600 & 0 & $\begin{array}{l}\text { Initial Decant } \\
\text { 2AY/6C }\end{array}$ \\
\hline & $\mathrm{X}$ & & $\begin{array}{l}\text { WASH- } \\
\text { WATER }\end{array}$ & AY-102 & $3 / 30 / 05$ & $4 / 1 / 05$ & 324,000 & 0 & $\begin{array}{l}\text { First Wash. } \\
\text { 2AY/6C }\end{array}$ \\
\hline
\end{tabular}


Table A-1. Projected Transfers Through October 2011. (26 sheets)

Note: This table is extracted from Table $\mathrm{H}-1$ of the Tank Waste Remediation System

Operations and Utilization Plan (Kirkbride et al. 1997)

\begin{tabular}{|c|c|c|c|c|c|c|c|c|c|}
\hline $\begin{array}{l}\text { Boot } \\
\text { Strap }\end{array}$ & $\begin{array}{c}\text { HLW } \\
\text { Flag }\end{array}$ & $\begin{array}{l}\text { LAW } \\
\text { Flag }\end{array}$ & From & To & $\begin{array}{l}\text { Start } \\
\text { Date }\end{array}$ & $\begin{array}{l}\text { End } \\
\text { Date }\end{array}$ & $\begin{array}{c}\text { Liquid } \\
\text { (gallons) }\end{array}$ & $\begin{array}{c}\text { Solid } \\
\text { (Gallons) } \\
\end{array}$ & Notes \\
\hline & & $\mathbf{Y}$ & RETURN_1 & AP-107 & $4 / 25 / 05$ & $4 / 25 / 05$ & 8,680 & 328 & \begin{tabular}{|l|} 
St/TRU \& \\
Entrained Solids \\
Return from LAW \\
Contractor 1 \\
\end{tabular} \\
\hline . & & $\mathrm{Y}$ & RETURN_2 & AP-107 & $5 / 3 / 05$ & $5 / 3 / 05$ & 8,676 & 328 & $\begin{array}{l}\text { Entrained Solids } \\
\text { Return from LAW } \\
\text { Contractor } 2 \\
\end{array}$ \\
\hline & & $\mathbf{Y}$ & AP-106 & \begin{tabular}{|l|} 
LAW- \\
CONTRAC \\
TOR-1 \\
\end{tabular} & $3 / 20 / 05$ & $5 / 22 / 05$ & 277,000 & 558 & $\begin{array}{l}\text { Process Envelope } \\
\text { B Batch } \\
\text { Contractor } 1 \\
\end{array}$ \\
\hline & & Y & AP-108 & $\begin{array}{l}\text { LAW- } \\
\text { CONTRAC } \\
\text { TOR-2 } \\
\end{array}$ & $3 / 21 / 05$ & $-5 / 23 / 05$ & 273,000 & 550 & $\begin{array}{l}\text { Process Envelope } \\
\text { B Batch } \\
\text { Contractor } 2 \\
\end{array}$ \\
\hline & & & $\mathrm{AN}-105$ & AW-102 & $5 / 21 / 05$ & $5 / 25 / 05$ & 772,500 & 13,977 & \\
\hline & $Y$ & & AY-102 & AN-105 & $5 / 20 / 05$ & $5 / 26 / 05$ & 302,200 & 0 & $\begin{array}{l}\text { Firrst Wash } \\
\text { Decant 2AY/6C }\end{array}$ \\
\hline & $\mathbf{Y}$ & & $\begin{array}{l}\text { WASH- } \\
\text { WATER }\end{array}$ & AY-102 & $5 / 27 / 05$ & $5 / 29 / 05$ & 324,000 & 0 & $\begin{array}{l}\text { Second Wash } \\
2 \mathrm{AY} / 6 \mathrm{C}\end{array}$ \\
\hline & & & AW-102 & $242-A$ & $6 / 1 / 05$ & $6 / 5 / 05$ & 872,800 & 31 & 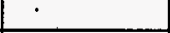 \\
\hline & & & $242-A$ & AW-106 & $6 / 1 / 05$ & $6 / 5 / 05$ & 121,900 & 31 & \\
\hline & & & 242-A & LERF & $6 / 1 / 05$ & $6 / 5 / 05$ & 750,900 & 0 & \\
\hline & & & $\mathrm{AN}-105$ & AW-102 & $6 / 10 / 05$ & $6 / 12 / 05$ & 390,600 & 14 & \\
\hline & & & AW-106 & AW-101 & $7 / 1 / 05$ & $7 / 1 / 05$ & 121,700 & 6 & \\
\hline & & $Y$ & AP-102 & AP-106 & $7 / 13 / 05$ & $7 / 14 / 05$ & 170,400 & 184 & $\begin{array}{l}\text { Delíver LAW } \\
\text { Batch 6, } \\
\text { Contractor 1, } \\
\text { Envelope C } \\
\end{array}$ \\
\hline & & $\mathbf{Y}$ & AP-104 & AP-108 & $7 / 16 / 05$ & $7 / 17 / 05$ & 170,500 & 186 & \begin{tabular}{|l} 
Deliver LAW \\
Batch 6, \\
Contractor 2, \\
Envelope C \\
\end{tabular} \\
\hline & $Y$ & & AY -102 & AN-105 & $7 / 17 / 05$ & $7 / 19 / 05$ & 322,100 & 0 & $\begin{array}{l}\text { Second Wash } \\
\text { Decant } 2 \mathrm{AY} / 6 \mathrm{C}\end{array}$ \\
\hline & $Y$ & & $\begin{array}{l}\text { WASH- } \\
\text { WATER }\end{array}$ & AY-102 & $7 / 20 / 05$ & $7 / 22 / 05$ & 559,000 & 0 & $\begin{array}{l}\text { Transfer Water } \\
\text { Add 2AY/6C }\end{array}$ \\
\hline & & $Y$ & RETURN_1 & AP-107 & $8 / 3 / 05$ & $8 / 4 / 05$ & 8,701 & 305 & \begin{tabular}{|l|} 
Sr/TRU \& \\
Entrained Solids \\
Return from LAW \\
Contractor 1 \\
\end{tabular} \\
\hline & $\mathbf{Y}$ & & $\begin{array}{l}\text { PIHLW_FE } \\
\text { ED_TANKK }\end{array}$ & $\begin{array}{l}\text { HLW- } \\
\text { CONTRAC } \\
\text { TOR }\end{array}$ & $9 / 27 / 04$ & $8 / 4 / 05$ & 145,900 & 6,032 & $\begin{array}{l}\text { Process Batch } 2 \\
\text { of } 2 \mathrm{AZ} \text {, Envelope } \\
\text { D (Batch } 4 \\
\text { Overall) }\end{array}$ \\
\hline & $Y$ & & $A Z-102$ & $\begin{array}{l}\text { P1HLW_F } \\
\text { EED_TAN } \\
\text { K }\end{array}$ & $8 / 4 / 05$ & $8 / 5 / 05$ & 145,900 & 6,032 & $\begin{array}{l}\text { Deliver Batch } 3 \text { of } \\
\text { 2AZ, Envelope D } \\
\text { (Batch } 5 \text { Overall) }\end{array}$ \\
\hline
\end{tabular}


Table A-1. Projected Transfers Through October 2011. (26 sheets)

Note: This table is extracted from Table $\mathrm{H}-1$ of the Tank Waste Remediation System

Operations and Utilization Plan (Kirkbride et al. 1997)

\begin{tabular}{|c|c|c|c|c|c|c|c|c|c|}
\hline $\begin{array}{l}\text { Boot } \\
\text { Strap } \\
\end{array}$ & $\begin{array}{l}\text { HLW } \\
\text { Flag }\end{array}$ & $\begin{array}{l}\text { LAW } \\
\text { Flag }\end{array}$ & From & To & $\begin{array}{l}\text { Start } \\
\text { Date } \\
\end{array}$ & $\begin{array}{l}\text { End } \\
\text { Date } \\
\end{array}$ & $\begin{array}{c}\text { Liquid } \\
\text { (gallons) }\end{array}$ & $\begin{array}{c}\text { Solid } \\
\text { (Gallons) } \\
\end{array}$ & Notes \\
\hline & & $\mathbf{Y}$ & AP-102 & AP-106 & $8 / 10 / 05$ & $8 / 12 / 05$ & 389,000 & 420 & $\begin{array}{l}\text { Deliver LAW } \\
\text { Batch 7, } \\
\text { Contractor 1, } \\
\text { Envelope C } \\
\end{array}$ \\
\hline & & $\mathrm{Y}$ & AP-104 & AP-108 & $8 / 13 / 05$ & $8 / 15 / 05$ & 389,100 & 426 & $\begin{array}{l}\text { Deliver LAW } \\
\text { Batch 7, } \\
\text { Contractor 2, } \\
\text { Envelope C } \\
\end{array}$ \\
\hline & & $Y$ & AN-102 & AP-102 & $8 / 13 / 05$ & $8 / 16 / 05$ & 481,700 & 23 & $\begin{array}{l}\text { Retrieve/Stage } \\
\text { LAW Batch } 8 \text {, } \\
\text { Contractor 1, } \\
\text { Envelope C } \\
\end{array}$ \\
\hline & & Y & LERF & $\begin{array}{l}\text { AN-102- } \\
\text { PUMP }\end{array}$ & $8 / 13 / 05$ & $8 / 16 / 05$ & 289,000 & 0 & Dilution Water \\
\hline & & $Y$ & AN-102 & AP-102 & $8 / 13 / 05$ & $8 / 16 / 05$ & 770,700 & 23 & As-received Ghost \\
\hline & & $\mathbf{Y}$ & AN-102 & AP-104 & $8 / 17 / 05$ & $8 / 20 / 05$ & 481,700 & 23 & $\begin{array}{l}\text { Retrieve/Stage } \\
\text { LAW Batch } 8 \text {, } \\
\text { Contractor 2, } \\
\text { Envelope C }\end{array}$ \\
\hline & . & $\mathbf{Y}$ & LERF & $\begin{array}{l}\text { AN-102- } \\
\text { PUMP } \\
\end{array}$ & $8 / 17 / 05$ & $8 / 20 / 05$ & 289,000 & 0 & Dilution Water \\
\hline & & $Y$ & $\mathrm{AN}-102$ & AP-104 & $8 / 17 / 05$ & $8 / 20 / 05$ & 770,700 & 23 & As-received Ghost \\
\hline & & $Y$ & RETURN_2 & AP-107 & $9 / 2 / 05$ & $9 / 2 / 05$ & 8,672 & 329 & $\begin{array}{l}\text { Entrained Solids } \\
\text { Return from LAW } \\
\text { Contractor } 2\end{array}$ \\
\hline & & & WESF & $\mathrm{AN}-105$ & $9 / 17 / 05$ & $9 / 17 / 05$ & 5,000 & 0 & \\
\hline & & & TNS88 & $\mathrm{AN}-105$ & $9 / 17 / 05$ & $9 / 17 / 05$ & 750 & 250 & \\
\hline & & & TAL88 & AN-105 & 9/17/05 & 9/17/05 & 24,000 & 0 & \\
\hline & & & $34 \mathrm{~L} 87$ & AN-105 & $9 / 17 / 05$ & $9 / 17 / 05$ & 50,000 & 0 & \\
\hline & & & WATER & AN-105 & $9 / 17 / 05$ & $9 / 17 / 05$ & 35,000 & 0 & \\
\hline & & $\therefore$ & WATER & AN-105 & $9 / 17 / 05$ & $9 / 17 / 05$ & 5,000 & 0 & \\
\hline & & & EVAPF & AN-105 & $9 / 17 / 05$ & 9/17/05 & 120,000 & 0 & . \\
\hline & & & WATER & AN-105 & $9 / 18 / 05$ & $9 / 18 / 05$ & 22,000 & 0 & $:$ \\
\hline & & & EVAPF & AW-102 & $9 / 20 / 05$ & 9/20/05 & 35,000 & 0 & \\
\hline & & $\mathbf{Y}$ & RETURN_1 & AP-107 & $10 / 15 / 05$ & $10 / 15 / 05$ & 8,774 & 234 & $\begin{array}{l}\text { Sr/TRU \& } \\
\text { Entrained Solids } \\
\text { Return from LAW } \\
\text { Contractor } 1\end{array}$ \\
\hline & & & AW-102 & $242-A$ & $11 / 22 / 05$ & $11 / 25 / 05$ & 425,600 & 15 & \\
\hline & & & $242-A$ & AW-106 & $11 / 22 / 05$ & $11 / 25 / 05$ & 51,529 & 15 & \\
\hline & & & $242-A$ & LERF & $11 / 22 / 05$ & $11 / 25 / 05$ & 374,100 & 0 & \\
\hline & & & AW-102 & 242-A & $12 / 1 / 05$ & $12 / 1 / 05$ & 0 & 0 & \\
\hline & & & $242-A$ & AW-106 & $12 / 1 / 05$ & $12 / 1 / 05$ & 아 & 와 & \\
\hline & & & $242-\mathrm{A}$ & LERF & $12 / 1 / 05$ & $12 / 1 / 05$ & 아 & 와 & \\
\hline & & & AN-105 & AW-102 & $12 / 10 / 05$ & $12 / 12 / 05$ & 581,100 & 20 & \\
\hline
\end{tabular}


Table A-1. Projected Transfers Through October 2011. (26 sheets)

Note: This table is extracted from Table H-1 of the Tank Waste Remediation System

Operations and Utilization Plan (Kirkbride et al. 1997)

\begin{tabular}{|c|c|c|c|c|c|c|c|c|c|}
\hline $\begin{array}{l}\text { Boot } \\
\text { Strap }\end{array}$ & $\begin{array}{c}\text { HLW } \\
\text { Flag }\end{array}$ & $\begin{array}{l}\text { LAW } \\
\text { Flag }\end{array}$ & From & To & $\begin{array}{l}\text { Start } \\
\text { Date }\end{array}$ & $\begin{array}{l}\text { End } \\
\text { Date }\end{array}$ & $\begin{array}{c}\text { Liquid } \\
\text { (gallons) }\end{array}$ & $\begin{array}{c}\text { Solid } \\
\text { (Gallons) }\end{array}$ & Notes \\
\hline & & $Y$ & RETURN_2 & AP-107 & $12 / 13 / 05$ & $12 / 13 / 05$ & 8,673 & 330 & \begin{tabular}{|l|} 
Entrained Solids \\
Return from LAW \\
Contractor 2
\end{tabular} \\
\hline & & & AW-105 & AN-105 & $12 / 15 / 05$ & $12 / 18 / 05$ & 713,900 & 25 & \\
\hline & & $Y$ & RETURN_1 & AP-107 & $12 / 27 / 05$ & $12 / 27 / 05$ & 8,778 & 232 & \begin{tabular}{|l|} 
Sr/TRU \& \\
Entrained Solids \\
Return from LAW \\
Contractor 1 \\
\end{tabular} \\
\hline & & $\mathbf{Y}$ & AP-102 & AP-106 & $12 / 24 / 05$ & $12 / 27 / 05$ & 770,700 & 51 & \begin{tabular}{|l} 
Deliver LAW \\
Batch 8, \\
Contractor 1, \\
Envelope C
\end{tabular} \\
\hline & & $Y$ & AN-106 & AP-102 & $12 / 28 / 05$ & $12 / 31 / 05$ & 547,500 & 24 & $\begin{array}{l}\text { Retrieve/Stage } \\
\text { LAW Batch 9, } \\
\text { Contractor 1, } \\
\text { Envelope C }\end{array}$ \\
\hline & & $\mathbf{Y}$ & LERF & $\begin{array}{l}\text { AN-106- } \\
\text { PUMP }\end{array}$ & $12 / 28 / 05$ & $12 / 31 / 05$ & 98,555 & 0 & Dilution Water \\
\hline & & $Y$ & AN-106 & AP-102 & $12 / 28 / 05$ & $12 / 31 / 05$ & 646,100 & 24 & As-received Ghost \\
\hline & & $\mathrm{Y}$ & AP-104 & AP-108 & $12 / 27 / 05$ & $12 / 31 / 05$ & 770,700 & 51 & $\begin{array}{l}\text { Deliver LAW } \\
\text { Batch 8, } \\
\text { Contractor 2, } \\
\text { Envelope C }\end{array}$ \\
\hline & & & AW-106 & AW-101 & $1 / 1 / 06$ & $1 / 1 / 06$ & 51,422 & 2 & \\
\hline & & $\mathbf{X}$ & $S Y-102$ & AW-105 & $1 / 1 / 06$ & $1 / 3 / 06$ & 580,400 & 22,129 & $\begin{array}{l}\text { Cleanout Solids in } \\
2 \mathrm{SY}\end{array}$ \\
\hline & & $\mathbf{Y}$ & AN-106 & AP-104 & $1 / 1 / 06$ & $1 / 4 / 06$ & 547,500 & 24 & $\begin{array}{l}\text { Retrieve/Stage } \\
\text { LAW Batch 9, } \\
\text { Contractor 2, } \\
\text { Envelope C }\end{array}$ \\
\hline & & $\mathbf{Y}$ & LERF & $\begin{array}{l}\text { AN-106- } \\
\text { PUMP }\end{array}$ & $1 / 1 / 06$ & $1 / 4 / 06$ & 98,555 & 0 & Dilution Water \\
\hline & & $Y$ & AN-106 & AP-104 & $1 / 1 / 06$ & $1 / 4 / 06$ & 646,100 & 24 & As-received Ghost \\
\hline & & & AW-105 & AN-105 & $3 / 1 / 06$ & $3 / 2 / 06$ & 321,000 & 11 & \\
\hline & & & ZNL87 & SY-102 & $3 / 20 / 06$ & $3 / 20 / 06$ & 3,000 & 0 & \\
\hline & & & WATER & SY-102 & $3 / 20 / 06$ & $3 / 20 / 06$ & 6,000 & 0 & \\
\hline & & & SPN87 & SY-102 & $3 / 20 / 06$ & $3 / 20 / 06$ & 25,000 & 0 & $\cdot$ \\
\hline & & & AN-103 & AW-105 & $4 / 2 / 06$ & $4 / 4 / 06$ & 458,900 & 12,056 & \\
\hline & & $\mathbf{Y}$ & SY-101 & SY-102 & $4 / 1 / 06$ & $4 / 6 / 06$ & 537,500 & 5,484 & $\begin{array}{l}\text { Retrieve/Stage } \\
\text { LAW Batch } 10\end{array}$ \\
\hline & & $\mathrm{X}$ & LERF & $\begin{array}{l}\text { SY-101- } \\
\text { PUMP }\end{array}$ & $4 / 1 / 06$ & $4 / 6 / 06$ & 532,100 & 0 & Dilution Water \\
\hline & & $Y$ & SY-101 & SY-102 & $4 / 1 / 06$ & $4 / 6 / 06$ & $1,082,000$ & 611 & As-received Ghost \\
\hline & & & AN-103 & AN-106 & $4 / 4 / 06$ & $4 / 6 / 06$ & 390,600 & 10,249 & \\
\hline & & $\mathbf{Y}$ & SY-102 & AN-102 & $4 / 7 / 06$ & $4 / 10 / 06$ & $1,027,000$ & 1,457 & $\begin{array}{l}\text { Cross-site/Pre- } \\
\text { stage LAW Batch } \\
10\end{array}$ \\
\hline
\end{tabular}


Table A-1. Projected Transfers Through October 2011. (26 sheets)

Note: This table is extracted from Table H-1 of the Tank Waste Remediation System

Operations and Utilization Plan (Kirkbride et al. 1997)

\begin{tabular}{|c|c|c|c|c|c|c|c|c|c|}
\hline $\begin{array}{l}\text { Boot } \\
\text { Strap } \\
\end{array}$ & $\begin{array}{l}\text { HLW } \\
\text { Flag }\end{array}$ & $\begin{array}{l}\text { LAW } \\
\text { Flag } \\
\end{array}$ & From & To & $\begin{array}{l}\text { Start } \\
\text { Date }\end{array}$ & $\begin{array}{l}\text { End } \\
\text { Date }\end{array}$ & $\begin{array}{c}\text { Liquid } \\
\text { (gallons) }\end{array}$ & $\begin{array}{c}\text { Solid } \\
\text { (Gallons) } \\
\end{array}$ & Notes \\
\hline & & $\mathbf{Y}$ & SY-101 & SY-102 & $4 / 11 / 06$ & $4 / 16 / 06$ & 510,600 & 5,209 & $\begin{array}{l}\text { Retrieve/Stage } \\
\text { LAW Batch } 10\end{array}$ \\
\hline & & $\mathbf{Y}$ & LERF & $\begin{array}{l}\text { SY-101- } \\
\text { PUMP }\end{array}$ & $4 / 11 / 06$ & $4 / 16 / 06$ & 505,500 & 0 & Dilution Water \\
\hline & & $\mathbf{Y}$ & $S Y-101$ & SY-102 & $4 / 11 / 06$ & $4 / 16 / 06$ & $1,028,000$ & 580 & As-received Ghost \\
\hline & & $\dot{Y}$ & SY-102 & AN-107 & $4 / 17 / 06$ & $4 / 20 / 06$ & 983,800 & 640 & $\begin{array}{l}\text { Cross-site/Pre- } \\
\text { stage LAW Batch } \\
10\end{array}$ \\
\hline & & $\mathrm{Y}$ & SY-101 & SY-102 & $4 / 21 / 06$ & $4 / 21 / 06$ & 27,388 & 279 & $\begin{array}{l}\text { Retrieve/Stage } \\
\text { LAW Batch } 11\end{array}$ \\
\hline & & $\mathrm{Y}$ & LERF & $\begin{array}{l}\text { SY-101- } \\
\text { PUMP }\end{array}$ & $4 / 21 / 06$ & $4 / 21 / 06$ & 27,114 & 0 & Dilution Water \\
\hline & & $\mathbf{Y}$ & SY-101 & SY-102 & $4 / 21 / 06$ & $4 / 21 / 06$ & 55,131 & 31 & As-received Ghost \\
\hline & & & AW-102 & $242-A$ & $6 / 1 / 06$ & $6 / 4 / 06$ & 581,100 & 20 & \\
\hline & & & $242-\mathrm{A}$ & AW-106 & $6 / 1 / 06$ & $6 / 4 / 06$ & 22,200 & 20 & $\therefore$ \\
\hline & & & $242-A$ & LERF & $6 / 1 / 06$ & $6 / 4 / 06$ & 558,900 & 0 & \\
\hline & & & $C-105$ & AN-103 & $11 / 30 / 04$ & $6 / 7 / 06$ & 891,200 & 31,875 & \\
\hline & $Y$ & & $\begin{array}{l}\text { PlHLW_FE } \\
\text { ED_TAÑK }\end{array}$ & $\begin{array}{l}\text { HLW- } \\
\text { CONTRAC } \\
\text { TOR }\end{array}$ & $8 / 5 / 05$ & $6 / 12 / 06$ & 145,900 & 6,032 & $\begin{array}{l}\text { Process Batch } 3 \\
\text { of 2AZ, Envelope } \\
\text { D (Batch } 5 \\
\text { Overall) } \\
\end{array}$ \\
\hline & $\mathrm{Y}$ & & AY-102 & $\begin{array}{l}\text { P1HLW_F } \\
\text { EED_TÃN } \\
K\end{array}$ & $6 / 12 / 06$ & $6 / 12 / 06$ & 135,000 & 3,416 & $\begin{array}{l}\text { Deliver Batch } 1 \text { of } \\
\text { 2AY/6C, } \\
\text { Envelope D (Batc } \\
6 \text { Overall) } \\
\end{array}$ \\
\hline & & Y & RETURN_1 & AP-107 & $6 / 13 / 06$ & $6 / 13 / 06$ & 8,902 & 102 & \begin{tabular}{|l|} 
St/TRU \& \\
Entrained Solids \\
Return from LAW \\
Contractor 1 \\
\end{tabular} \\
\hline & & & AN-105 & AW-102 & $6 / 10 / 06$ & $6 / 14 / 06$ & 872,900 & 30 & \\
\hline & & & AW-106 & AW-101 & $7 / 1 / 06$ & $7 / 1 / 06$ & 22,041 & 1 & \\
\hline & . & $Y$ & AP-102 & AP-106 & $8 / 20 / 06$ & $8 / 24 / 06$ & 646,100 & 25 & $\begin{array}{l}\text { Deliver LAW } \\
\text { Batch 9, } \\
\text { Contractor } 1, \\
\text { Envelope C }\end{array}$ \\
\hline & & $\mathbf{Y}$ & AP-104 & AP-108 & $8 / 24 / 06$ & $8 / 27 / 06$ & 646,100 & 25 & $\begin{array}{l}\text { Deliver LAW } \\
\text { Batch 9, } \\
\text { Contractor 2, } \\
\text { Envelope C } \\
\end{array}$ \\
\hline & & $\mathbf{Y}$ & SY-103 & SY-102 & $8 / 26 / 06$ & $8 / 28 / 06$ & 350,000 & 9,759 & $\begin{array}{l}\text { Retrieve/Stage } \\
\text { LAW Batch } 11 \\
\end{array}$ \\
\hline & & $\mathbf{Y}$ & LERF & $\begin{array}{l}\text { SY-103- } \\
\text { PUMP }\end{array}$ & $8 / 26 / 06$ & $8 / 28 / 06$ & 183,500 & 0 & Dilation Water \\
\hline & & $\mathrm{Y}$ & SY-103 & SY-102 & $8 / 26 / 06$ & $8 / 28 / 06$ & 537,500 & 1,449 & As-received Ghost \\
\hline & & $\mathbf{Y}$ & AN-102 & AP-102 & $8 / 25 / 06$ & $8 / 30 / 06$ & $1,014,000$ & 44 & $\begin{array}{l}\text { Stage LAW Batch } \\
10, \text { Contractor } 1, \\
\text { Envelope C }\end{array}$ \\
\hline
\end{tabular}


Table A-1. Projected Transfers Through October 2011. (26 sheets)

Note: This table is extracted from Table H-1 of the Tank Waste Remediation System

Operations and Utilization Plan (Kirkbride et al. 1997)

\begin{tabular}{|c|c|c|c|c|c|c|c|c|c|}
\hline $\begin{array}{l}\text { Boot } \\
\text { Strap }\end{array}$ & $\begin{array}{c}\text { HLW } \\
\text { Flag }\end{array}$ & $\begin{array}{l}\text { LAW } \\
\text { Flag }\end{array}$ & From & To & $\begin{array}{l}\text { Start } \\
\text { Date }\end{array}$ & $\begin{array}{l}\text { End } \\
\text { Date }\end{array}$ & $\begin{array}{c}\text { Liquid } \\
\text { (gallons) }\end{array}$ & $\begin{array}{c}\text { Solid } \\
\text { (Gallons) }\end{array}$ & Notes \\
\hline & & $\mathrm{Y}$ & SY-102 & AN-102 & $8 / 31 / 06$ & $9 / 2 / 06$ & 724,700 & 1,526 & $\begin{array}{l}\text { Cross-site/Pre- } \\
\text { stage LAW Batch } \\
11\end{array}$ \\
\hline & . & $Y$ & SY-103 & SY-102 & $9 / 3 / 06$ & $9 / 5 / 06$ & 350,000 & 9,759 & $\begin{array}{l}\text { Retrieve/Stage } \\
\text { LAW Batch } 11\end{array}$ \\
\hline & & $\mathrm{Y}$ & LERF & $\begin{array}{l}\text { SY-103- } \\
\text { PUMP }\end{array}$ & $9 / 3 / 06$ & $9 / 5 / 06$ & 183,500 & 0 & Dilution Water \\
\hline & & $Y$ & SY-103 & SY-102 & $9 / 3 / 06$ & 9/5/06 & 537,500 & 1,449 & As-received Ghost \\
\hline & & $\mathrm{Y}$ & AN-107 & AP-104 & $9 / 1 / 06$ & $9 / 5 / 06$ & 978,400 & 43 & \begin{tabular}{|l|} 
Stage LAW Batch \\
10, Contractor 2, \\
Envelope C
\end{tabular} \\
\hline & & $\mathbf{Y}$ & SY-102 & AN-107 & $9 / 6 / 06$ & $9 / 7 / 06$ & 537,500 & 1,434 & $\begin{array}{l}\text { Cross-site/Pre- } \\
\text { stage LAW Batch } \\
11\end{array}$ \\
\hline & & & $A-104$ & AN- 103 & $6 / 7 / 06$ & $9 / 15 / 06$ & 29,319 & 832 & \\
\hline & & & WESF & AN-105 & $9 / 17 / 06$ & $9 / 17 / 06$ & 5,000 & 0 & \\
\hline & & & TNS88 & AN-105 & $9 / 17 / 06$ & $9 / 17 / 06$ & 750 & 250 & \\
\hline & & & TAL88 & AN-105 & $9 / 17 / 06$ & $9 / 17 / 06$ & 25,000 & $\overline{0}$ & \\
\hline & & & WATER & AN-105 & $9 / 17 / 06$ & $9 / 17 / 06$ & 35,000 & 0 & \\
\hline & & & $34 \mathrm{~L} 87$ & AN-105 & $9 / 17 / 06$ & $9 / 17 / 06$ & 57,000 & 0 & \\
\hline & & & EVAPF & AN-105 & $9 / 17 / 06$ & $9 / 17 / 06$ & 120,000 & 0 & \\
\hline & & & WATER & AN-105 & $9 / 18 / 06$ & $9 / 18 / 06$ & 30,000 & 0 & \\
\hline & & & C-204 & AN-103 & $9 / 15 / 06$ & $9 / 22 / 06$ & 3,808 & 55 & \\
\hline & & & C-201 & AN-103 & $9 / 22 / 06$ & $9 / 28 / 06$ & 2,522 & 59 & \\
\hline & & & $\mathrm{AX}-104$ & AN-103 & $9 / 28 / 06$ & $11 / 3 / 06$ & 19,592 & 572 & \\
\hline & & & AW-102 & $242-\mathrm{A}$ & $12 / 1 / 06$ & $12 / 5 / 06$ & 872,900 & 30 & \\
\hline & & & $242-A$ & AW-106 & $12 / 1 / 06$ & $12 / 5 / 06$ & 75,050 & 30 & $\therefore$ \\
\hline & & & $242-A$ & LERF & $12 / 1 / 06$ & $12 / 5 / 06$ & 797,800 & 0 & \\
\hline & & & AN-105 & AW-102 & $12 / 10 / 06$ & $12 / 12 / 06$ & 431,900 & 15 & \\
\hline & & $\mathbf{Y}$ & RETURN_1 & AP-107 & $12 / 24 / 06$ & $12 / 24 / 06$ & 8,937 & 66 & \begin{tabular}{|l|} 
Sr/TRU \& \\
Entrained Solids \\
Return from LAW \\
Contractor 1 \\
\end{tabular} \\
\hline & & & AW-106 & AW-101 & $1 / 1 / 07$ & $1 / 1 / 07$ & 74,822 & 3 & \\
\hline & $\mathbf{Y}$ & & $\begin{array}{l}\text { P1HLW_FE } \\
\text { ED_TAÑK }\end{array}$ & $\begin{array}{l}\text { HLW- } \\
\text { CONTRAC } \\
\text { TOR }\end{array}$ & $6 / 12 / 06$ & $1 / 4 / 07$ & 135,000 & 3,416 & $\begin{array}{l}\text { Process Batch } 1 \\
\text { of } 2 \mathrm{AY} / 6 \mathrm{C} \text {, } \\
\text { Envelope D } \\
\text { (Batch 6 Overall) }\end{array}$ \\
\hline & $Y$ & & AY-102 & $\begin{array}{l}\text { P1HLW_F } \\
\text { EED_TÂN } \\
\text { K }\end{array}$ & $1 / 4 / 07$ & $1 / 4 / 07$ & 135,000 & 3,416 & \begin{tabular}{|l|} 
Deliver Batch 2 of \\
2AY/6C, \\
Envelope D \\
(Batch 7 Overall) \\
\end{tabular} \\
\hline & & & S-103 & SY-101 & $7 / 24 / 06$ & $1 / 15 / 07$ & 719,200 & 1,011 & \\
\hline & & & AX-102 & AN-103 & $11 / 3 / 06$ & $2 / 21 / 07$ & 88,610 & 897 & \\
\hline & & & $S X-113$ & SY-103 & $12 / 6 / 06$ & $3 / 13 / 07$ & 11,203 & 399 & \\
\hline
\end{tabular}


Table A-1. Projected Transfers Through October 2011. (26 sheets)

Note: This table is extracted from Table H-1 of the Tank Waste Remediation System

Operations and Utilization Plan (Kirkbride et al. 1997)

\begin{tabular}{|c|c|c|c|c|c|c|c|c|c|}
\hline $\begin{array}{l}\text { Boot } \\
\text { Strap } \\
\end{array}$ & $\begin{array}{c}\text { HLW } \\
\text { Flag } \\
\end{array}$ & $\begin{array}{c}\text { LAW } \\
\text { Flag }\end{array}$ & From & Tó & $\begin{array}{l}\text { Start } \\
\text { Date } \\
\end{array}$ & $\begin{array}{l}\text { End } \\
\text { Date }\end{array}$ & $\begin{array}{c}\text { Liquid } \\
\text { (gallons) }\end{array}$ & $\begin{array}{c}\text { Solid } \\
\text { (Gallons) }\end{array}$ & Notes \\
\hline & & $\mathbf{Y}$ & AP-102 & AP-106 & $3 / 13 / 07$ & $3 / 19 / 07$ & $1,014,000$ & 44 & \begin{tabular}{|l} 
Deliver LAW \\
Batch 10, \\
Contractor 1, \\
Envelope C \\
\end{tabular} \\
\hline & & & WATER & SY-102 & $3 / 20 / 07$ & $3 / 20 / 07$ & 5,000 & 0 & \\
\hline & & & SPN87 & SY-102 & $3 / 20 / 07$ & $3 / 20 / 07$ & 25,000 & 0 & \\
\hline & & $\mathbf{Y}$ & AN-102 & AP-102 & $3 / 19 / 07$ & $3 / 22 / 07$ & 711,200 & 31 & \begin{tabular}{|l|} 
Stage LAW Batch \\
11,Contractor 1, \\
Envelope C
\end{tabular} \\
\hline & & $\mathbf{Y}$ & AP-104 & AP-108 & $3 / 19 / 07$ & $3 / 23 / 07$ & 978,400 & 43 & $\begin{array}{l}\text { Deliver LAW } \\
\text { Batch 10, } \\
\text { Contractor 2, } \\
\text { Envelope C } \\
\end{array}$ \\
\hline & & $Y$ & AN-107 & AP-104 & $3 / 24 / 07$ & $3 / 26 / 07$ & 524,700 & 23 & $\begin{array}{l}\text { Stage LAW Batch } \\
11 \text {, Contractor 2, } \\
\text { Envelope C } \\
\end{array}$ \\
\hline & & & SY-102 & AW-102 & $4 / 4 / 07$ & $4 / 4 / 07$ & 29,195 & 1 & \\
\hline & & & AN-103 & AN-106 & $5 / 23 / 07$ & $5 / 26 / 07$ & 685,000 & 13,746 & \\
\hline & & & AW-102 & $242-\mathrm{A}$ & $6 / 1 / 07$ & $6 / 3 / 07$ & 461,100 & 16 & \\
\hline & & & $242-A$ & AW-106 & $6 / 1 / 07$ & $.6 / 3 / 07$ & 33,509 & 16 & \\
\hline & & & $242-\mathrm{A}$ & LERF & $6 / 1 / 07$ & $6 / 3 / 07$ & 427,600 & 0 & \\
\hline & & & AN-105 & AW-102 & $6 / 10 / 07$ & $6 / 10 / 07$ & 0 & 0 & \\
\hline & & & SY-101 & AN-102 & $6 / 20 / 07$ & $6 / 25 / 07$ & 997,400 & 1,678 & \\
\hline & & & AN-103 & AN-107 & $6 / 24 / 07$ & $6 / 26 / 07$ & 410,600 & 8,230 & \\
\hline & & & AN-102 & AN-107 & $6 / 26 / 07$ & $6 / 29 / 07$ & 541,200 & 4,267 & \\
\hline & & & AW-106 & AW-101 & $7 / 1 / 07$ & $7 / 1 / 07$ & 33,387 & 2 & \\
\hline & & & $\mathrm{SX}-112$ & $S Y-103$ & $3 / 13 / 07$ & $7 / 23 / 07$ & 485,300 & 16,406 & \\
\hline & $\mathbf{Y}$ & & $\begin{array}{l}\text { P1HLW_FE } \\
\text { ED_TANKK }\end{array}$ & \begin{tabular}{|l|} 
HLW- \\
CONTRAC \\
TOR
\end{tabular} & $1 / 4 / 07$ & $7 / 29 / 07$ & 135,000 & 3,416 & $\begin{array}{l}\text { Process Batch } 2 \\
\text { of } 2 \mathrm{AY} / 6 \mathrm{C}, \\
\text { Envelope D } \\
\text { (Batch } 7 \text { Overall) } \\
\end{array}$ \\
\hline & & $\mathbf{Y}$ & RETURN_1 & AP-107 & $7 / 29 / 07$ & $7 / 29 / 07$ & 8,967 & 36 & \begin{tabular}{|l|} 
Sr/TRU \& \\
Entrained Solids \\
Return from LAW \\
Contractor 1 \\
\end{tabular} \\
\hline & $\mathbf{Y}$ & & AY-102 & $\begin{array}{l}\text { P1HLW_F } \\
\text { EED_TÃN } \\
\text { K }\end{array}$ & $7 / 29 / 07$ & $7 / 29 / 07$ & 135,000 & 3,416 & $\begin{array}{l}\text { Deliver Batch } 3 \text { of } \\
\text { 2AY/6C, } \\
\text { Envelope D } \\
\text { (Batch 8 Overall) } \\
\end{array}$ \\
\hline & & & TNS88 & AN-105 & $9 / 17 / 07$ & $9 / 17 / 07$ & 750 & 250 & \\
\hline & & & TAL88 & AN-105 & $9 / 17 / 07$ & $9 / 17 / 07$ & 25,000 & 0 & \\
\hline & & & $34 \mathrm{~L} 87$ & AN-105 & $9 / 17 / 07$ & $9 / 17 / 07$ & 50,000 & 0 & \\
\hline & & & WATER & AN-105 & 9/17/07 & $9 / 17 / 07$ & 35,000 & 0 & \\
\hline & & & WESF & AN-105 & 9/17/07 & $9 / 17 / 07$ & 5,000 & 0 & \\
\hline & & & WATER & AN-105 & $9 / 17 / 07$ & $9 / 17 / 07$ & 27,000 & 0 & \\
\hline & & & EVAPF & AN- 105 & $9 / 17 / 07$ & $9 / 17 / 07$ & 120,000 & of & \\
\hline
\end{tabular}


HNF-2168

Revision 0

Table A-1. Projected Transfers Through October 2011. (26 sheets)

Note: This table is extracted from Table $\mathrm{H}-1$ of the Tank Waste Remediation System

Operations and Utilization Plan (Kirkbride et al. 1997)

\begin{tabular}{|c|c|c|c|c|c|c|c|c|c|}
\hline $\begin{array}{l}\text { Boot } \\
\text { Strap }\end{array}$ & $\begin{array}{l}\text { HLW } \\
\text { Flag }\end{array}$ & $\begin{array}{l}\text { LAW } \\
\text { Flag }\end{array}$ & From & To & $\begin{array}{l}\text { Start } \\
\text { Date }\end{array}$ & $\begin{array}{l}\text { End } \\
\text { Date }\end{array}$ & $\begin{array}{c}\text { Liquid } \\
\text { (gallons) }\end{array}$ & $\begin{array}{c}\text { Solid } \\
\text { (Gallons) }\end{array}$ & Notes \\
\hline & & & EVAPF & AW-102 & $9 / 20 / 07$ & $9 / 20 / 07$ & 35,000 & 0 & \\
\hline & & & SX-110 & SY-103 & $7 / 23 / 07$ & $11 / 25 / 07$ & 285,900 & 8,910 & \\
\hline & & & AW-102 & $242-\mathrm{A}$ & $12 / 1 / 07$ & $12 / 1 / 07$ & 35,013 & 1 & \\
\hline & & & $242-\mathrm{A}$ & AW-106 & $12 / 1 / 07$ & $12 / 1 / 07$ & 1,120 & 1 & \\
\hline & & & 242-A & LERF & $12 / 1 / 07$ & $12 / 1 / 07$ & 33,893 & 0 & \\
\hline & & & AN-105 & AW-102 & $12 / 10 / 07$ & $12 / 11 / 07$ & 259,900 & 9 & \\
\hline & & & AW-106 & AW-101 & $1 / 1 / 08$ & $1 / 1 / 08$ & 1,111 & 0 & \\
\hline & & $\mathbf{Y}$ & AP-102 & AP-106 & $1 / 16 / 08$ & $1 / 19 / 08$ & 711,200 & 31 & \begin{tabular}{|l} 
Deliver LAW \\
Batch 11, \\
Contractor 1, \\
Envelope C \\
\end{tabular} \\
\hline & & $Y$ & AP-104 & AP-108 & $1 / 19 / 08$ & $1 / 22 / 08$ & 524,700 & 23 & $\begin{array}{l}\text { Deliver LAW } \\
\text { Batch 11, } \\
\text { Contractor 2, } \\
\text { Envelope C } \\
\end{array}$ \\
\hline & & & SX-115 & SY-103 & $11 / 25 / 07$ & $1 / 26 / 08$ & 51,011 & 1,557 & \\
\hline & $\mathbf{Y}$ & & $\begin{array}{l}\text { P1HLW_FE } \\
\text { ED_TAÑK }\end{array}$ & $\begin{array}{l}\text { HLW- } \\
\text { CONTRAC } \\
\text { TOR }\end{array}$ & $7 / 29 / 07$ & $2 / 20 / 08$ & 135,000 & 3,416 & $\begin{array}{l}\text { Process Batch } 3 \\
\text { of 2AY/6C, } \\
\text { Envelope D } \\
\text { (Batch 8 Overall) } \\
\end{array}$ \\
\hline & $Y$ & & AY -102 & $\begin{array}{l}\text { P1HLW_F } \\
\text { EED_TAN } \\
\text { K }\end{array}$ & $2 / 20 / 08$ & $2 / 20 / 08$ & 135,000 & 3,416 & $\begin{array}{l}\text { Deliver Batch } 4 \text { of } \\
\text { 2AY } / 6 \mathrm{C}, \\
\text { Envelope D } \\
\text { (Batch } 9 \text { Overall) } \\
\end{array}$ \\
\hline & & $\mathbf{Y}$ & RETURN_1 & AP-107 & $3 / 12 / 08$ & $3 / 12 / 08$ & 8,968 & 33 & $\begin{array}{l}\text { St/TRU \& } \\
\text { Entrained Solids } \\
\text { Return from LAW } \\
\text { Contractor 1 } \\
\end{array}$ \\
\hline & & & WATER & SY-102 & $3 / 20 / 08$ & $3 / 20 / 08$ & 5,000 & 0 & - \\
\hline & & & SPN87 & SY-102 & $3 / 20 / 08$ & $3 / 20 / 08$ & 25,000 & 0 & . \\
\hline & & & SY-102 & AW-102 & $4 / 4 / 08$ & $4 / 4 / 08$ & 29,999 & 1 & \\
\hline & & & AW-102 & $242-A$ & $6 / 1 / 08$ & $.6 / 2 / 08$ & 289,900 & 10 & \\
\hline & & & 242-A & AW-106 & $6 / 1 / 08$ & $6 / 2 / 08$ & 12,379 & 10 & \\
\hline & & . & 242-A & LERF & $6 / 1 / 08$ & $6 / 2 / 08$ & 277,500 & 0 & \\
\hline & & & AN-105 & AW-102 & $6 / 10 / 08$ & $6 / 10 / 08$ & 0 & 0 & \\
\hline & & & AW-106 & AW-101 & $7 / 1 / 08$ & $7 / 1 / 08$ & 12,300 & 1 & \\
\hline & & $Y$ & AP-108 & $\begin{array}{l}\text { LAW- } \\
\text { CONTRAC } \\
\text { TOR-2 } \\
\end{array}$ & $7 / 17 / 05$ & $8 / 3 / 08$ & $3,479,000$ & 809 & $\begin{array}{l}\text { Process Envelope } \\
\text { C Batches } \\
\text { Contractor } 2 \\
\end{array}$ \\
\hline & & $Y$ & RETURN_2 & AP-107 & $8 / 4 / 08$ & $8 / 4 / 08$ & 8,149 & 310 & $\begin{array}{l}\text { Entrained Solids } \\
\text { Return from LAW } \\
\text { Contractor } 2 \\
\end{array}$ \\
\hline & $\mathbf{Y}$ & & $\begin{array}{l}\text { P1HLW_FE } \\
\text { ED_TANKK }\end{array}$ & $\begin{array}{l}\text { HLW- } \\
\text { CONTRAC } \\
\text { TOR }\end{array}$ & $2 / 20 / 08$ & $9 / 13 / 08$ & 135,000 & 3,416 & $\begin{array}{l}\text { Process Batch } 4 \\
\text { of } 2 \mathrm{AY} / 6 \mathrm{C} \text {, } \\
\text { Envelope D } \\
\text { (Baich } 9 \text { Overall) }\end{array}$ \\
\hline
\end{tabular}


HNF-2168

Revision 0

Table A-1. Projected Transfers Through October 2011. (26 sheets)

Note: This table is extracted from Table $\mathrm{H}-1$ of the Tank Waste Remediation System

Operations and Utilization Plan (Kirkbride et al. 1997)

\begin{tabular}{|c|c|c|c|c|c|c|c|c|c|}
\hline $\begin{array}{l}\text { Boot } \\
\text { Strap }\end{array}$ & $\begin{array}{c}\text { HLW } \\
\text { Flag }\end{array}$ & $\begin{array}{c}\text { LAW } \\
\text { Flag } \\
\end{array}$ & From & To & $\begin{array}{l}\text { Start } \\
\text { Date }\end{array}$ & $\begin{array}{l}\text { End } \\
\text { Date }\end{array}$ & $\begin{array}{c}\text { Liquid } \\
\text { (gallons) }\end{array}$ & $\begin{array}{c}\text { Solid } \\
\text { (Gallons) } \\
\end{array}$ & Notes \\
\hline & $\mathbf{Y}$ & & $A Y-102$ & $\begin{array}{l}\text { P1HLW_F } \\
\text { EED_TAN } \\
\text { K }\end{array}$ & $9 / 13 / 08$ & $9 / 13 / 08$ & 135,000 & 3,416 & $\begin{array}{l}\text { Deliver Batch } 5 \text { of } \\
2 \mathrm{AY} / 6 \mathrm{C}, \\
\text { Envelope D } \\
\text { (Batch } 10 \text { Overall) } \\
\end{array}$ \\
\hline & & & TNS88 & AN-105 & 9/17/08 & $9 / 17 / 08$ & 750 & 250 & \\
\hline & & & WATER & AN-105 & $9 / 17 / 08$ & $9 / 17 / 08$ & 5,000 & 0 & \\
\hline & & & TAL88 & AN-105 & 9/17/08 & $9 / 17 / 08$ & 25,000 & 0 & \\
\hline & & & WESF & AN-105 & $9 / 17 / 08$ & $9 / 17 / 08$ & 5,000 & 0 & \\
\hline & & & WATER & AN-105 & $9 / 17 / 08$ & $9 / 17 / 08$ & 35,000 & 0 & \\
\hline & & & $34 \mathrm{~L} 87$ & AN-105 & $9 / 17 / 08$ & $9 / 17 / 08$ & 50,000 & 0 & \\
\hline & & & EVAPF & AN-105 & $9 / 17 / 08$ & $9 / 17 / 08$ & 120,000 & 0 & \\
\hline & & & WATER & $\mathrm{AN}-105$ & $9 / 18 / 08$ & $9 / 18 / 08$ & 22,000 & 0 & \\
\hline & & & EVAPF & AW-102 & $9 / 20 / 08$ & $9 / 20 / 08$ & 85,000 & 0 & \\
\hline & & $\mathbf{Y}$ & AP-106 & \begin{tabular}{|l|} 
LAW- \\
CONTRAC \\
TOR-1 \\
\end{tabular} & $7 / 14 / 05$ & $9 / 27 / 08$ & $3,701,000$ & 810 & \begin{tabular}{|l|} 
Process Envelope \\
C Batches \\
Contractor 1 \\
\end{tabular} \\
\hline & & $\mathbf{Y}$ & RETURN_1 & AP-107 & $9 / 28 / 08$ & $9 / 28 / 08$ & 7,048 & 28 & \begin{tabular}{|l|} 
Sr/TRU \& \\
Entrained Solids \\
Return from LAW \\
Contractor 1 \\
\end{tabular} \\
\hline & & & AP-107 & AZ-101 & $10 / 9 / 08$ & $10 / 10 / 08$ & 338,400 & 10,357 & \\
\hline & & & AN-103 & AP-107 & $10 / 20 / 08$ & $10 / 25 / 08$ & $1,113,000$ & 3,571 & \\
\hline & & & $A W-102$ & $242-\mathrm{A}$ & $12 / 1 / 08$ & $12 / 1 / 08$ & 85,032 & 3 & \\
\hline & & & $242-\mathrm{A}$ & AW-106 & $12 / 1 / 08$ & $12 / 1 / 08$ & 891 & 3 & \\
\hline & & & $242-\mathrm{A}$ & LERF & $12 / 1 / 08$ & $12 / 1 / 08$ & 84,141 & 0 & \\
\hline & & & AN-105 & $\mathrm{AW}-102$ & $12 / 10 / 08$ & $12 / 11 / 08$ & $\therefore \quad 259,900$ & 9 & \\
\hline & & & AW-106 & AW-101 & $1 / 1 / 09$ & $1 / 1 / 09$ & 867 & 0 & \\
\hline & & & U-202 & UA-1 & $1 / 4 / 09$ & $1 / 11 / 09$ & 29,792 & 1,061 & \\
\hline & & & $\mathrm{U}-203$ & UA-1 & $1 / 11 / 09$ & $1 / 17 / 09$ & 15,084 & 538 & \\
\hline & & & $\mathrm{AX}-101$ & AN-103 & $2 / 21 / 07$ & $2 / 26 / 09$ & $2,167,000$ & 6,072 & \\
\hline & & & U-110 & UA-1 & $1 / 17 / 09$ & $2 / 27 / 09$ & 100,200 & 3,312 & $\dot{.}$ \\
\hline & & & UA-1 & SY-102 & $2 / 28 / 09$ & $3 / 1 / 09$ & 145,500 & 4,907 & \\
\hline & & & C-202 & $\mathrm{AN}-103$ & $2 / 26 / 09$ & $3 / 3 / 09$ & 1,208 & 28 & \\
\hline & & & WATER & SY-102 & $3 / 20 / 09$ & $3 / 20 / 09$ & 5,000 & 0 & \\
\hline & & & SPN87 & SY-102 & $3 / 20 / 09$ & $3 / 20 / 09$ & 25,000 & 0 & \\
\hline & & & SY-102 & AW-102 & $4 / 4 / 09$ & 4/4/09 & 29,999 & 1 &. \\
\hline & $\mathbf{Y}$ & & $\begin{array}{l}\text { P1HLW_FE } \\
\text { ED_TANKK }\end{array}$ & $\begin{array}{l}\text { HLW- } \\
\text { CONTRAC } \\
\text { TOR }\end{array}$ & $9 / 13 / 08$ & $4 / 7 / 09$ & 135,000 & 3,416 & $\begin{array}{l}\text { Process Batch } 5 \\
\text { of 2AY/6C, } \\
\text { Envelope D } \\
\text { (Batch 10 Overall) } \\
\end{array}$ \\
\hline & $Y$ & & AY-102 & $\mid \begin{array}{l}\text { P1HLW_F } \\
\text { EED_TAN } \\
K\end{array}$ & $4 / 7 / 09$ & $4 / 7 / 09$ & 135,000 & 3,416 & $\begin{array}{l}\text { Deliver Batch } 6 \text { of } \\
\text { 2AY/6C, } \\
\text { Envelope D } \\
\text { Batch } 11 \text { Overalt) }\end{array}$ \\
\hline
\end{tabular}


Table A-1. Projected Transfers Through October 2011. (26 sheets)

Note: This table is extracted from Table $\mathrm{H}-1$ of the Tank Waste Remediation System

Operations and Utilization Plan (Kirkbride et al. 1997)

\begin{tabular}{|c|c|c|c|c|c|c|c|c|c|}
\hline $\begin{array}{l}\text { Boot } \\
\text { Strap } \\
\end{array}$ & $\begin{array}{c}\text { HLW } \\
\text { Flag } \\
\end{array}$ & $\begin{array}{c}\text { LAW } \\
\text { Flag } \\
\end{array}$ & From & To & $\begin{array}{l}\text { Start } \\
\text { Date } \\
\end{array}$ & $\begin{array}{l}\text { End } \\
\text { Date }\end{array}$ & $\begin{array}{c}\text { Liquid } \\
\text { (gallons) }\end{array}$ & $\begin{array}{c}\begin{array}{c}\text { Solid } \\
\text { (Gallons) }\end{array} \\
\end{array}$ & Notes \\
\hline & & & U-110 & UA-2 & $2 / 27 / 09$ & $4 / 29 / 09$ & 145,200 & 4,798 & \\
\hline & & & UA-2 & SY-102 & $4 / 30 / 09$ & $5 / 1 / 09$ & 145,600 & 4,795 & \\
\hline & & & A-105 & AN-103 & $3 / 3 / 09$ & $5 / 26 / 09$ & 52,980 & 1,545 & \\
\hline & & & $\mathrm{U}-110$ & UA-1 & $4 / 29 / 09$ & $5 / 31 / 09$ & 75,945 & 2,510 & \\
\hline & & & C-203 & AN-103 & $5 / 26 / 09$ & $6 / 2 / 09$ & 6,429 & 151 & \\
\hline & & & AW-102 & 242-A & $6 / 1 / 09$ & $6 / 2 / 09$ & 289,900 & 10 & \\
\hline & & & $242-\mathrm{A}$ & AW-106 & $6 / 1 / 09$ & $6 / 2 / 09$ & 10,702 & 10 & \\
\hline & & & $242-\mathrm{A}$ & LERF & $6 / 1 / 09$ & $6 / 2 / 09$ & 279,200 & 0 & \\
\hline & & & AN-105 & $\mathrm{AW}-102$ & $6 / 10 / 09$ & $6 / 10 / 09$ & 0 & 0 & \\
\hline & & & AW-106 & AW-101 & $7 / 1 / 09$ & $7 / 1 / 09$ & 10,622 & 0 & - \\
\hline & & & U-101 & UA-1 & $5 / 31 / 09$ & $7 / 30 / 09$ & 28,629 & 668 & \\
\hline & & & C-108 & AN-103 & $6 / 2 / 09$ & $8 / 18 / 09$ & 121,700 & 3,842 & \\
\hline & & & WESF & AN-105 & $9 / 17 / 09$ & $9 / 17 / 09$ & 5,000 & 0 & \\
\hline & & & WATER & AN-105 & $9 / 17 / 09$ & 9/17/09 & 35,000 & 0 & \\
\hline & & & $34 \mathrm{~L} 87$ & AN-105 & $9 / 17 / 09$ & $9 / 17 / 09$ & 57,000 & 0 & \\
\hline & & & WATER & AN-105 & $9 / 17 / 09$ & $9 / 17 / 09$ & 8,000 & 0 & \\
\hline & & & TNS88 & AN-105 & $9 / 17 / 09$ & 9/17/09 & 750 & 250 & \\
\hline & & & TAL 88 & AN-105 & $9 / 17 / 09$ & $9 / 17 / 09$ & 26,000 & 0 & $\cdot$ \\
\hline & & & EVAPF & AN-105 & $9 / 17 / 09$ & $9 / 17 / 09$ & 120,000 & 0 & \\
\hline & & & WATER & AN-105 & $9 / 18 / 09$ & $9 / 18 / 09$ & 22,000 & 0 & \\
\hline & & & EVAPF & AW-102 & $9 / 20 / 09$ & $9 / 20 / 09$ & 35,000 & 0 & \\
\hline & & & U-104 & UA-1 & $7 / 30 / 09$ & $10 / 20 / 09$ & 41,182 & 966 & \\
\hline & & & UA-1 & SY-102 & $10 / 21 / 09$ & $10 / 22 / 09$ & 146,300 & 4,144 & \\
\hline & $\mathbf{Y}$ & & $\begin{array}{l}\text { PlHLW_FE } \\
\text { ED_TAÑK }\end{array}$ & $\begin{array}{l}\text { HLW- } \\
\text { CONTRAC } \\
\text { TOR }\end{array}$ & $4 / 7 / 09$ & $10 / 30 / 09$ & 135,000 & 3,416 & $\begin{array}{l}\text { Process Batch } 6 \\
\text { of 2AY/6C, } \\
\text { Envelope D } \\
\text { (Batch 11 Overaill) }\end{array}$ \\
\hline & & & C-111 & AN-103 & $8 / 18 / 09$ & $10 / 30 / 09$ & 204,800 & 7,081 & \\
\hline & & & $\mathrm{U}-104$ & UA-2 & $10 / 20 / 09$ & $11 / 10 / 09$ & 10,889 & 255 & \\
\hline & & & AW-102 & 242-A & $12 / 1 / 09$ & $12 / 1 / 09$ & 35,013 & 1 & \\
\hline & & & $242-A$ & AW-106 & $12 / 1 / 09$ & $12 / 1 / 09$ & 570 & 1 & . \\
\hline & & & 242-A & LERF & $12 / 1 / 09$ & $12 / 1 / 09$ & 34,444 & 0 & \\
\hline & & & AN-105 & AW-102 & $12 / 10 / 09$ & $12 / 11 / 09$ & 270,900 & 9 & \\
\hline & & & U-112 & UA-2 & $11 / 10 / 09$ & $12 / 31 / 09$ & 134,100 & 4,664 & \\
\hline & & & AW-106 & AW-101 & $1 / 1 / 10$ & $1 / 1 / 10$ & 560 & 0 & \\
\hline & & & UA-2 & SY-102 & $1 / 1 / 10$ & $1 / 1 / 10$ & 145,500 & 4,919 & \\
\hline & & & U-112 & UA-1 & $12 / 31 / 09$ & $1 / 18 / 10$ & 49,643 & 1,727 & \\
\hline & & & $\mathrm{U}-204$ & UA-1 & $\mathrm{t} / 18 / 10$ & $1 / 24 / 10$ & 14,154 & 503 & \\
\hline & & & $\mathrm{U}-201$ & UA-1 & $1 / 24 / 10$ & $1 / 31 / 10$ & 29,794 & 1,061 & \\
\hline & & & U-106 & UA-1 & $1 / 31 / 10$ & $2 / 12 / 10$ & 52,886 & 131 & \\
\hline & & & UA-1 & SY-102 & $2 / 13 / 10$ & $2 / 14 / 10$ & 147,000 & 3,423 & \\
\hline & & & U-106 & UA-2 & $2 / 12 / 10$ & $3 / 17 / 10$ & 149,500 & 370 & \\
\hline
\end{tabular}


HNF-2168

Revision 0

Table A-1. Projected Transfers Through October 2011. (26 sheets)

Note: This table is extracted from Table $\mathrm{H}-1$ of the Tank Waste Remediation System

Operations and Utilization Plan (Kirkbride et al. 1997)

\begin{tabular}{|c|c|c|c|c|c|c|c|c|c|}
\hline $\begin{array}{l}\text { Boot } \\
\text { Strap } \\
\end{array}$ & $\begin{array}{c}\text { HLW } \\
\text { Flag } \\
\end{array}$ & $\begin{array}{l}\text { LAW } \\
\text { Flag } \\
\end{array}$ & From & To & $\begin{array}{l}\text { Start } \\
\text { Date } \\
\end{array}$ & $\begin{array}{l}\text { End } \\
\text { Date } \\
\end{array}$ & $\begin{array}{c}\text { Liquid } \\
\text { (gallons) }\end{array}$ & $\begin{array}{c}\text { Solid } \\
\text { (Gallons) }\end{array}$ & Notes \\
\hline & & & UA-2 & SY-102 & $3 / 18 / 10$ & $3 / 19 / 10$ & 150,000 & 373 & \\
\hline & & & WATER & SY-102 & $3 / 20 / 10$ & $3 / 20 / 10$ & 5,000 & 0 & \\
\hline & & & SPN87 & SY-102 & $3 / 20 / 10$ & $3 / 20 / 10$ & 25,000 & 0 & \\
\hline & & & SY-102 & AW-102 & $4 / 4 / 10$ & $4 / 4 / 10$ & 29,999 & 1 & \\
\hline & & & U-106 & UA-1 & $3 / 17 / 10$ & $4 / 19 / 10$ & 149,500 & 370 & \\
\hline & & & U-106 & UA-2 & $4 / 19 / 10$ & $5 / 22 / 10$ & $\$ 49,500$ & 370 & \\
\hline & & & AW-102 & $242-\mathrm{A}$ & $6 / 1 / 10$ & $6 / 2 / 10$ & 300,900 & 10 & . \\
\hline & & & $242-A$ & AW-106 & $6 / 1 / 10$ & $6 / 2 / 10$ & 16,159 & 10 & \\
\hline & & & 242-A & LERF & $6 / 1 / 10$ & $6 / 2 / 10$ & 284,700 & 0 & \\
\hline & & & AN-105 & AW-102 & $6 / 10 / 10$ & $6 / 10 / 10$ & 0 & 0 & \\
\hline & & & AW-106 & AW-101 & $7 / 1 / 10$ & $7 / 1 / 10$ & 16,078 & 1 & \\
\hline & & & WESF & AN-105 & $9 / 17 / 10$ & $9 / 17 / 10$ & 5,000 & 0 & . \\
\hline & & & WATER & SY-102 & $9 / 17 / 10$ & $9 / 17 / 10$ & 5,000 & 0 & \\
\hline & & & WATER & AN-105 & $9 / 17 / 10$ & $9 / 17 / 10$ & 35,000 & 0 & \\
\hline & & & WATER & AN-105 & $9 / 17 / 10$ & $9 / 17 / 10$ & 25,000 & 0 & \\
\hline & & & SPN87 & SY-102 & $9 / 17 / 10$ & $9 / 17 / 10$ & 25,000 & 0 & \\
\hline & & & TNS88 & AN-105 & $9 / 17 / 10$ & $9 / 17 / 10$ & 750 & 250 & \\
\hline & & 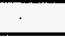 & WATER & AN-105 & $9 / 17 / 10$ & $9 / 17 / 10$ & 22,000 & 0 & \\
\hline & & & TAL88 & AN-105 & $9 / 17 / 10$ & $9 / 17 / 10$ & 26,000 & 0 & \\
\hline & & & $34 \mathrm{~L} 87$ & AN-105 & $9 / 17 / 10$ & $9 / 17 / 10$ & 50,000 & 0 & \\
\hline & & & EVAPF & AN-105 & $9 / 17 / 10$ & $9 / 18 / 10$ & 120,000 & 0 & \\
\hline & & & WATER & AN-105 & $9 / 18 / 10$ & $9 / 18 / 10$ & 5,000 & 0 & \\
\hline & & & EVAPF & AW-102 & $9 / 20 / 10$ & $9 / 20 / 10$ & 85,000 & 0 & \\
\hline & & & EVAPF & AW-102 & $10 / 19 / 10$ & $10 / 19 / 10$ & 35,000 & 0 & \\
\hline & . & & AW-102 & $242-A$ & $12 / 1 / 10$ & $12 / 2 / 10$ & 120,000 & 4 & \\
\hline & & & $242-\mathrm{A}$ & AW-106 & $12 / 1 / 10$ & $12 / 2 / 10$ & 1,207 & 4 & \\
\hline & & & $242-A$ & LERF & $12 / 1 / 10$ & $12 / 2 / 10$ & 118,800 & 0 & \\
\hline & & & AN-105 & AW-102 & $12 / 10 / 10$ & $12 / 11 / 10$ & 285,900 & 10 & \\
\hline & & & AW-106 & AW-101 & $1 / 1 / 11$ & $1 / 1 / 11$ & 1,173 & 0 & \\
\hline & & & TX-109 & TA-2 & $1 / 4 / 11$ & $2 / 4 / 11$ & 145,100 & 4,929 & $\cdot$ \\
\hline & & & BY-110 & $B A-3$ & $1 / 4 / 11$ & $2 / 8 / 11$ & 147,400 & 2,619 & \\
\hline & & & TY-101 & TA-3 & $1 / 4 / 11$ & $2 / 26 / 11$ & 146,300 & 3,722 & \\
\hline & & & T-106 & TA-1 & $1 / 4 / 11$ & $.3 / 3 / 11$ & 83,358 & 2,929 & \\
\hline & & & TX-109 & TA-4 & $2 / 4 / 11$ & $3 / 7 / 11$ & 145,100 & 4,929 & \\
\hline & & & B-112 & BA-1 & $1 / 4 / 11$ & $3 / 9 / 11$ & 73,902 & 1,083 & . \\
\hline & & & BY-110 & $\mathrm{BA}-4$ & $2 / 8 / 11$ & $3 / 16 / 11$ & 147,400 & 2,619 & \\
\hline & & & WATER & AN-105 & $3 / 20 / 11$ & $3 / 20 / 11$ & 50,000 & 0 & \\
\hline & & & $\mathrm{T}-101$ & TA-1 & $3 / 3 / 11$ & $3 / 25 / 11$ & 61,500 & 2,213 & \\
\hline & & & SY-102 & AW-102 & $4 / 4 / 11$ & $4 / 4 / 11$ & 29,360 & 640 & \\
\hline & & & BX-102 & BA-2 & $1 / 4 / 11$ & $4 / 4 / 11$ & 77,293 & 2,445 & \\
\hline & & & TX-109 & TA-6 & $3 / 7 / 11$ & $4 / 8 / 11$ & 145,100 & 4,929 & \\
\hline
\end{tabular}


Table A-1. Projected Transfers Through October 2011. (26 sheets)

Note: This table is extracted from Table H-1 of the Tank Waste Remediation System

Operations and Utilization Plan (Kirkbride et al. 1997)

\begin{tabular}{|c|c|c|c|c|c|c|c|c|c|}
\hline $\begin{array}{l}\text { Boot } \\
\text { Strap }\end{array}$ & $\begin{array}{c}\text { HLW } \\
\text { Flag }\end{array}$ & $\begin{array}{l}\text { LAW } \\
\text { Flag }\end{array}$ & From & To & $\begin{array}{l}\text { Start } \\
\text { Date }\end{array}$ & $\begin{array}{l}\text { End } \\
\text { Date }\end{array}$ & $\begin{array}{c}\text { Liquid } \\
\text { (gallons) }\end{array}$ & $\begin{array}{c}\text { Solid } \\
\text { (Gallons) }\end{array}$ & Notes \\
\hline & & & B-111 & BA-1 & $3 / 9 / 11$ & $4 / 8 / 11$ & 72,735 & 2,279 & \\
\hline & & & TY-101 & TA-5 & $2 / 26 / 11$ & $4 / 21 / 11$ & 146,300 & 3,722 & \\
\hline & & & BY-110 & BA-5 & $3 / 16 / 11$ & $4 / 21 / 11$ & 147,400 & 2,619 & \\
\hline & & & BX-112 & BA-2 & $4 / 4 / 11$ & $4 / 26 / 11$ & 68,037 & 2,225 & \\
\hline & & & $\mathrm{BA}-3$ & AP-102 & $6 / 3 / 11$ & $6 / 3 / 11$ & 154,800 & 2,618 & \\
\hline & & & BA-4 & AP-106 & $6 / 3 / 11$ & $6 / 3 / 11$ & 154,800 & 2,618 & \\
\hline & & & BA-1 & AP-102 & $6 / 3 / 11$ & $6 / 4 / 11$ & 154,000 & 3,360 & \\
\hline & & & BA-5 & AP-106 & $6 / 3 / 11$ & $6 / 4 / 11$ & 154,800 & 2,618 & \\
\hline & & & BA-2 & AP-102 & $6 / 4 / 11$ & $6 / 5 / 11$ & 152,700 & 4,666 & \\
\hline & & & AN-102 & AP-108 & $6 / 3 / 11$ & $6 / 5 / 11$ & 567600 & 4471.731 & \\
\hline & & & AN-103 & AP-104 & $6 / 3 / 11$ & $6 / 8 / 11$ & 1100000 & 16309.103 & \\
\hline & & & AN-103 & AP-108 & $6 / 8 / 11$ & $6 / 8 / 11$ & 1286.432 & 18.73 & \\
\hline & & & B-111 & BA-6 & $4 / 8 / 11$ & $6 / 8 / 11$ & 145400 & 4557.783 & \\
\hline & & . & $\mathrm{BA}-6$ & AP-108 & $6 / 9 / 11$ & $6 / 10 / 11$ & 152800 & 4554.889 & \\
\hline & & & AN-105 & AW-102 & $6 / 10 / 11$ & $6 / 10 / 11$ & 50019.843 & 1.671 & \\
\hline & & & SY-103 & AN-102 & $6 / 6 / 11$ & $6 / 11 / 11$ & 1081000 & 35221.667 & \\
\hline & & & AN-102 & AP-108 & $6 / 12 / 11$ & $6 / 14 / 11$ & 373800 & 11943.632 & \\
\hline & & & WATER & AN-105 & $6 / 16 / 11$ & $6 / 16 / 11$ & 25000 & 0 & . \\
\hline & & & AN-102 & AP-102 & $6 / 14 / 11$ & $6 / 17 / 11$ & 624400 & 19936.032 & \\
\hline & & & $\mathrm{AN}-102$ & AP-106 & $6 / 17 / 11$ & $6 / 17 / 11$ & 85245.764 & 2709.747 & \\
\hline & & & SY-101 & AN-102 & $6 / 18 / 11$ & $6 / 23 / 11$ & 1115000 & 1794.438 & \\
\hline & & & AN-102 & AP-106 & $6 / 24 / 11$ & $6 / 27 / 11$ & 712100 & 1647.42 & \\
\hline & & & AW-106 & AW-101 & $7 / 1 / 11$ & $7 / 1 / 11$ & 0.005 & $2.292 \mathrm{E}-07$ & \\
\hline & & & BY-110 & $\mathrm{BA}-4$ & $6 / 3 / 11$ & $7 / 9 / 11$ & 147300 & 2617.469 & \\
\hline & & & S-105 & SY-101 & $1 / 15 / 07$ & $7 / 20 / 11$ & 1537000 & 2458.692 & \\
\hline & & & BX-112 & BA-3 & $6 / 3 / 11$ & $7 / 20 / 11$ & 145200 & 4746.215 & \\
\hline & & & B-111 & BA-1 & $6 / 8 / 11$ & $8 / 7 / 11$ & 145300 & 4554.744 & \\
\hline & & & BY-110 & BA-5 & $7 / 9 / 11$ & $8 / 14 / 11$ & 147300 & 2617.469 & \\
\hline & & & B-111 & BA- 6 & $8 / 7 / 11$ & $8 / 15 / 11$ & 17236.868 & 540.159 & \\
\hline & & & BX-112 & BA-2 & $7 / 20 / 11$ & 9/4/11 & 145200 & 4746.215 & \\
\hline & & & A-106 & AN-103 & $10 / 30 / 09$ & 9/4/11 & 259600 & 5796.036 & \\
\hline & & & $\mathrm{sX}-111$ & SY-103 & $1 / 26 / 08$ & $9 / 5 / 11$ & 606700 & 19768.212 & \\
\hline & & & BY-110 & BA-6 & $8 / 15 / 11$ & 9/15/11 & 129800 & 2307.057 & \\
\hline
\end{tabular}


Table A-2. Waste Sources Names and Definitions for Table A-1.

\begin{tabular}{|l|l|}
\hline 1FL96 & 105-F,105-H, and 100-N Liquid TCO Waste \\
\hline 1KL96 & 100-K Liquid TCO Waste \\
\hline 1NS96 & 100-Area Solid TCO Waste \\
\hline 242-A & 242-A Evaporator \\
\hline 34L87 & 300/400 Area Lab Waste \\
\hline BA-X & Annex Tanks for NE Quadrant \\
\hline BPTCO & B Plant TCO Waste \\
\hline EVAPF & Evaporator Flush and Tank Farm Water \\
\hline HLW-CONTRACTOR & HLW Contractor Processing Facility \\
\hline LAW-CONTRACTOR-X & LAW Contractor Processing Facility \\
\hline LERF & Evaporator Overheads Accumulation \\
\hline P1HLW_FEED_TANK & Phase 1 HLW Contractor Feed Tank \\
\hline PXTCO & PUREX TCO Waste \\
\hline RETURN_X & Phase 1 LAW Contractor Returns Tanks \\
\hline SPN87 & S Plant Dilute Non-Complexed Waste \\
\hline TA-X & Annex Tanks for NW Quadrant \\
\hline TAL88 & T Plant Supernate \\
\hline TNS88 & T Plant Solids \\
\hline UA-X & Annex Tanks for U Farm \\
\hline UNKN & Unknown Addition (Water) \\
\hline WASH-CAUSTIC & Caustic Solution for Enhanced Wash \\
\hline WASH-WATER & Dilute Caustic Solution for Solids Washing \\
\hline WATER & Water \\
\hline WCE88 & East Area Complexed Salt Well Liquid \\
\hline WCW88 & West Area Complexed Salt Well Liquid \\
\hline WESF & WESF Waste \\
\hline WNE88 & East Area Dilute, Non-Complexed Salt Well Liquid \\
\hline WNW88 & West Area Dilute, Non-Complexed Salt Well Liquid \\
\hline ZNL87 & Combined PFP Waste Stream \\
\hline
\end{tabular}


HNF-2168

Revision 0

A.2 READINESS TO PROCEED UPDATE TO TWRSO\&UP INFORMATION

Tables A-3 and A-4 were extracted from the Tank Waste Remediation System Retrieval and Disposal Mission Initial Updated Baseline Summary (Swita 1998). 
HNF-2168

Revision 0

This page intentionally left blank. 


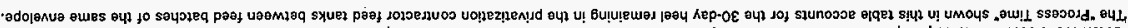

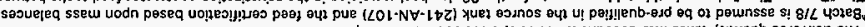

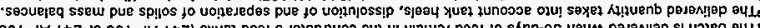

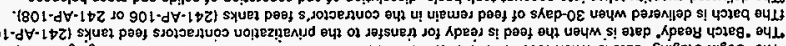

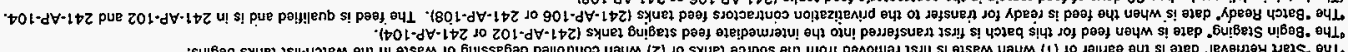

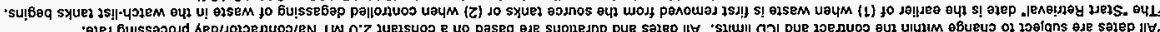

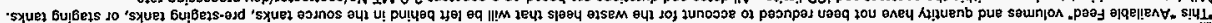

\begin{tabular}{|c|c|c|c|c|c|c|c|c|c|c|c|c|c|c|c|c|c|}
\hline \multirow{2}{*}{$06^{\circ}$} & \multirow{2}{*}{$68 L$} & $20-21$ & $66 \mathrm{~L}$ & $6 \cdot z$ & $60^{\circ} z$ & $\measuredangle \varepsilon \varepsilon$ & $800 \mathrm{Z} / \mathrm{L}$ & $800 Z / 9$ & \multirow{2}{*}{$2002 / 11$} & \multirow{2}{*}{$900 \mathrm{Z} / 21$} & \multirow{2}{*}{$\begin{array}{c}(800 Z f E) \\
L O L-N \forall-L Z Z \\
Z O L \cdot N \forall-L b Z\end{array}$} & $\angle 9^{\circ} 1$ & $6 \angle 2$ & $81 L$ & 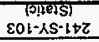 & \multirow[t]{2}{*}{$Z L$} & \multirow{9}{*}{0} \\
\hline & & $10-21$ & $99 z$ & $8 \cdot \varepsilon$ & $18^{\prime} \mathrm{Z}$ & 295 & $8002 / 6$ & $800 Z / \varepsilon$ & & & & \multirow{3}{*}{$\angle \varepsilon^{*} \downarrow$} & \multirow{3}{*}{$8 \tau^{\circ} t$} & \multirow{3}{*}{$06 \varepsilon L$} & \multirow{3}{*}{$\begin{array}{c}\text { (ग!le)S } \\
10 L^{-} \wedge S^{-} L t Z\end{array}$} & & \\
\hline \multirow{2}{*}{$\$ 9 \%$} & \multirow{2}{*}{$0 \& Z^{\prime} \downarrow$} & $20-11$ & $90 \varepsilon$ & 29 & $28 \varepsilon$ & S19 & $800 z / t$ & 2002101 & \multirow{2}{*}{$\angle 00 z / t$} & \multirow{2}{*}{$900 Z / Z 1$} & \multirow{2}{*}{$\begin{array}{c}\text { (900Z/G) } \\
\angle 01-N \forall-1 \text { L } \\
Z 01-N \forall-1 t Z\end{array}$} & & & & & \multirow{2}{*}{ LI } & \\
\hline & & $10-11$ & 908 & 29 & $28 \cdot \varepsilon$ & 519 & $\angle 002 / L 1$ & $\angle 00 Z / 8$ & & & & & & & & & \\
\hline 01.9 & $2 z 8$ & $\frac{25-01}{10-01}$ & $\frac{\angle O Z}{\angle O Z}$ & $\frac{g^{\prime} \varepsilon}{g^{\prime} \varepsilon}$ & $\frac{99^{2} 2}{99^{2} 2}$ & $\frac{I L t}{I L t}$ & $\frac{200 Z / 9}{\angle 00 Z / t / t}$ & $\begin{array}{c}\angle 00 Z / \varepsilon \\
900 Z / Z !\end{array}$ & $900 Z / 8$ & $900 z / 8$ & - & 560 & $\tau \varepsilon^{\prime} \downarrow$ & $9+8$ & $\begin{array}{c}16661 / 2) \\
901-N \forall-1+Z Z\end{array}$ & ot & \\
\hline 26.9 & $t 96$ & $\frac{25-6}{15-6}$ & $\frac{0+2}{0+2}$ & $\frac{T}{1+5}$ & $\frac{96^{2} Z}{96^{2} z}$ & $\frac{L L t}{L L t}$ & $\frac{900210 L}{9002 / 8}$ & $\begin{array}{c}900 z / 0 \mathrm{t} \\
9002 / 8\end{array}$ & $900 z / 7$ & $900 z / 7$ & - & $2 g^{\circ} z$ & 107 & 0901 & $\begin{array}{c}\text { (Jileis) } \\
z 0 t-N \forall-l t z\end{array}$ & 6 & \\
\hline $8 \varepsilon " \varepsilon$ & $\not g$ & $20-8$ & LEt & $\varepsilon^{2}$ & 691 & 212 & 900219 & $9002 / 8$ & & & & & & & & 8 & \\
\hline 86 & $D t g$ & $15-8$ & $\angle \varepsilon !$ & $\varepsilon^{\prime} z$ & 69.1 & $\overline{Z L Z}$ & $900 Z / t$ & $900 \mathrm{~d} / \mathrm{L}$ & $9002 / 1$ & $900 z / 1$ & - & $t t^{\prime} t$ & $97 \downarrow$ & 816 & $(2661 / 41)$ & & \\
\hline $8+1$ & $8 \varepsilon z$ & $\frac{20 \cdot L}{10 \cdot L}$ & $\frac{6 z}{6 z}$ & $\frac{01}{0.6}$ & $\frac{7 L O}{D L O}$ & $\frac{6 L L}{6 L t}$ & $\begin{array}{l}900279 \\
9002 / \varepsilon\end{array}$ & $\frac{900 Z / E}{900 Z / 1}$ & Sover & 9006 & & +5 & 56 & & $\angle O l-N \forall \cdot l \cdot \nabla Z$ & $u L$ & \\
\hline & & $20 \cdot 9$ & 89 & $\$ 1$ & 70.6 & 911 & $900 Z / \varepsilon$ & S00Z/8 & (0007/9 & (0007/0 & $200 z / 1\}$ & - & $\iota L^{\circ}$ & $\angle 61$ & $201-Z \forall-1 \nabla Z$ & & \\
\hline 600 & $\nabla 26$ & $10-9$ & 69 & $\nabla 1$ & $50^{\circ} 1$ & 811 & $9002 / 1$ & sooz/8 & 300010 & 900010 & $10 L-\lambda \theta$ & - & $2 ! \varepsilon$ & 698 & $101-z \gamma-1+z$ & 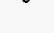 & \\
\hline & & & & & & & & & & & & $=$ & 280 & $08 \varepsilon$ & wịपs & & \\
\hline $8 \varepsilon-9$ & $9 \angle Z^{\prime} \downarrow$ & $20-5$ & เรE & 8 & LS' $\varepsilon$ & $10 L$ & gooz/E & $000 z / 11$ & $\$ 00 z / 9$ & $+00 z / 9$ & - & - & $\varepsilon 8^{\prime} \varepsilon$ & $16 t$ & $\begin{array}{c}16661 / 01) \\
+01-M \forall-1+2\end{array}$ & $\mathrm{~s}$ & \\
\hline & & 10.9 & 882 & $\varepsilon^{\circ} \varepsilon$ & $\angle 8 Z$ & $s<s$ & Gooz/E & $+00 z / 01$ & & & & $s \div 0$ & 81.7 & $5 * 2$ & $\begin{array}{c}16661 / 0) \\
101-d \forall \cdot 1+2 z\end{array}$ & & \\
\hline $9 \varepsilon^{\prime} 9$ & $0<1^{\prime} t$ & $\frac{25-t}{10-t}$ & $\frac{21 \varepsilon}{\angle 1 \varepsilon}$ & $\frac{t t}{t t}$ & $\begin{array}{l}85^{\prime} \varepsilon \\
8 !^{\circ} \varepsilon\end{array}$ & $\begin{array}{l}989 \\
989\end{array}$ & $\frac{700 \mathrm{Z} / \mathrm{s}}{700 \mathrm{~d} / \mathrm{s}}$ & $\frac{700 \mathrm{C} / \varepsilon}{500 \mathrm{Z} / \varepsilon}$ & $\varepsilon 00 z / 01$ & $\varepsilon 00 \mathrm{z} / \mathrm{s}$ & - & $\varepsilon 0^{\circ} \varepsilon$ & $69^{\circ} \varepsilon$ & tEZI & $\begin{array}{c}\text { (गDERS) } \\
\varepsilon 0 \mathrm{l}-\mathrm{N} \forall-\mathrm{I} \text { (t) }\end{array}$ & $\forall$ & $\forall$ \\
\hline$\tau L \mathcal{G}$ & 998 & $\frac{25-\varepsilon}{10-\varepsilon}$ & $\begin{array}{l}812 \\
812\end{array}$ & $\frac{6 \varepsilon}{6^{*} \varepsilon}$ & $\frac{98 Z}{98 z}$ & $\frac{824}{827}$ & $\frac{800210 \mathrm{~L}}{8002 / 01}$ & $\frac{800 z / 6}{\varepsilon 00 z / 8}$ & $\varepsilon 00 z / \varepsilon$ & $\varepsilon 00 z / t$ & $\cdot$ & $8 \sigma^{\circ} Z$ & 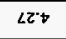 & 168 & $\begin{array}{c}\text { (J)ES) } \\
101-M V-1+z\end{array}$ & $\varepsilon$ & \\
\hline & $0 \% 0^{\prime} 1$ & $25-2$ & 992 & 8 & $I I^{\prime} Z$ & GEG & $800 E \pi$ & $2002 / 9$ & $100 z / z$ & $1002 / 0 \mathrm{~b}$ & - & $26: 1$ & $\measuredangle \iota \varepsilon$ & 0011 & (गneis) & $z$ & \\
\hline$\$ 9^{\circ} 9$ & $020^{\prime} 1$ & $10-z$ & 992 & $8 \cdot \varepsilon$ & $\angle L Z$ & SES & $\varepsilon 00 Z / 1$ & $2002 / 9$ & LOOETCL & 100eJub & 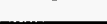 & $20 \%$ & 268 & 8011 & tol-N $\forall-1+2$ & & \\
\hline$g \tau s$ & $\angle Z O^{\prime} \mathrm{I}$ & $\frac{23-1}{15-1}$ & $\frac{G Z 2}{2 Z 2}$ & $\begin{array}{l}g^{\prime} \varepsilon \\
g^{\prime} \varepsilon \\
\end{array}$ & $\frac{29^{\prime 2}}{89^{\circ} z}$ & $\frac{819}{+19}$ & $\begin{array}{l}1002 / 2 ! \\
100 z / 21\end{array}$ & $\frac{100 z / Z !}{100 Z / Z 1}$ & $100 Z / 9$ & $100 Z / \varepsilon$ & - & $s+1$ & El't & 0601 & $\begin{array}{c}\text { (J)e } \\
\text { sol-N) }\end{array}$ & 1 & \\
\hline $\begin{array}{c}\text { (7w) } \\
\text { exunion }\end{array}$ & $\begin{array}{c}\text { (IW) } \\
\text { wn!Pos }\end{array}$ & dolvenuos & $\begin{array}{c}\text { (skeG) } \\
\text { row! } \\
\text { ssooodd }\end{array}$ & 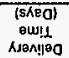 & 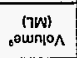 & $\begin{array}{c}(I W) \\
\text { oungpos }\end{array}$ & $\begin{array}{l}\text { pasenj|ap } \\
\text { 4ग1е8 }\end{array}$ & $\begin{array}{l}\text { Apees } \\
\text { पगेes }\end{array}$ & 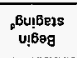 & $\begin{array}{l}\text { Jene!nad } \\
\text { LeIS }\end{array}$ & (OLEP DILELS) & $(7 w)$ & $\underset{\text { อwn| }}{(7 \mathrm{~W})}$ & $\underset{\text { wn!pos }}{(1 w)}$ & $\begin{array}{l}\text { (exep opress) } \\
\text { yue } 1 \text { oanos }\end{array}$ & 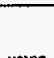 & \\
\hline 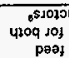 & $\begin{array}{l}\text { Duos } \\
\text { osen!lep } \\
101\end{array}$ & 4วखeg & נO? & SUOO पगR & 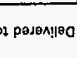 & peed & & 8u & dixoudd $\forall$ & & ebets-ald & 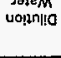 & & poos $\theta$ & eAv & पेder & oud porat \\
\hline
\end{tabular}

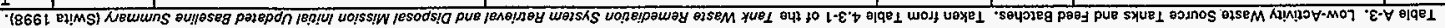




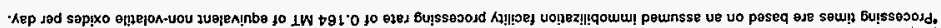

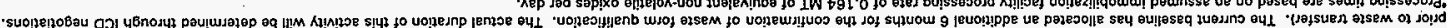
列

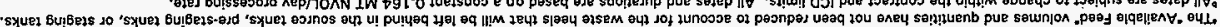

\begin{tabular}{|c|c|c|c|c|c|c|c|c|c|c|c|c|c|c|c|c|c|}
\hline$\varepsilon+1$ & I & $\angle Z{ }^{\circ} 0$ & $s+\varepsilon z$ & \multirow{3}{*}{ SE.। } & \multirow{3}{*}{$\angle L$} & $6002 / 01$ & \multirow{3}{*}{$900 z / s$} & \multirow{3}{*}{$900 z / L$} & \multirow{3}{*}{ sooz/L } & \multirow{3}{*}{$+002 / L$} & \multirow{3}{*}{$z 0 L-Z \forall$} & \multirow{3}{*}{ 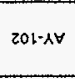 } & \multirow{3}{*}{$z \mathbf{l} \cdot \mathbf{b}$} & \multirow{3}{*}{$0.98 \varepsilon$} & \multirow{3}{*}{001.0} & \multirow{2}{*}{$\frac{z b}{b 1}$} & \multirow{3}{*}{ 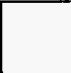 } \\
\hline$\$ 8 z$ & 1 & $t 9^{\prime} 0$ & 6.95 & & & $800 Z / 2 \mathrm{~L}$ & & & & & & & & & & & \\
\hline 982 & $!$ & $\rightarrow 50$ & 6.95 & & & $800 z / \varepsilon$ & & & & & & & & & & ol & \\
\hline $00 z$ & 1 & 670 & $6.2 \varepsilon$ & \multirow{4}{*}{$s t z$} & \multirow{4}{*}{$99 t$} & $\angle 002 / 8$ & \multirow{4}{*}{$+00 z / t$} & \multirow{4}{*}{$\varepsilon 00 z / 9$} & \multirow{4}{*}{$800 z / 9$} & \multirow{4}{*}{$866 \mathrm{~W} / \mathrm{O}$} & \multirow{4}{*}{$10 L-Z V$} & \multirow{4}{*}{ zOL-גל } & \multirow{2}{*}{ SLO } & \multirow{4}{*}{$\begin{array}{l}\varepsilon^{*} 99 \downarrow \\
g^{\prime} \angle \varepsilon\end{array}$} & \multirow{4}{*}{$\begin{array}{l}90 t-0 \\
\text { zot-גy }\end{array}$} & 6 & \multirow{8}{*}{0} \\
\hline $00 z$ & I & $6+0$ & 628 & & & $900 Z / L$ & & & & & & & & & & $L$ & \\
\hline 002 & 1 & $6+0$ & 628 & & & $900 z / 1$ & & & & & & & 80.0 & & & 9 & \\
\hline 002 & $t$ & $60^{\circ} 0$ & 6.78 & & & s00z/9 & & & & & & & & & & s & \\
\hline 962 & 1 & S90 & 9.87 & \multirow{2}{*}{0.1} & \multirow{2}{*}{$0^{\prime}<6$} & $5002 / 8$ & \multirow{2}{*}{$800 z / z$} & \multirow{2}{*}{$200 z / t$} & \multirow{2}{*}{ - } & \multirow{2}{*}{ - } & \multirow{2}{*}{$201-28$} & \multirow{2}{*}{ - } & 60 & 6.191 & $701 \rightarrow 78$ & $t$ & \\
\hline $96 z$ & 1 & SG'o & $5^{\prime} 8 t$ & & & E00Z/L! & & & & & & & 680 & 5196 & 20620 & $\varepsilon$ & \\
\hline$\$ 92$ & 1 & $\varepsilon S^{\prime} 0$ & $t \varepsilon t$ & 90.1 & 8.98 & $\varepsilon 00 z / 2$ & 100218 & 0007101 & - & . & & - & & & & 2 & \\
\hline 692 & 1 & $89^{\circ} 0$ & $\forall \varepsilon$ & 901 & $8^{-9} 98$ & $2002 / 9$ & $100 z / 8$ & $000 z / 0 \mathrm{l}$ & - & - & $10 t-28$ & - & 810 & $\forall 996$ & $10 t-2 \forall$ & 1 & \\
\hline $\begin{array}{c}\text { (SKep) } \\
\text { au! SSQSOId }\end{array}$ & 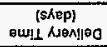 & $\begin{array}{c}(7 W) \\
\text { aunion }\end{array}$ & $\begin{array}{c}\text { unw } \\
\text { supueno }\end{array}$ & $\begin{array}{c}\text { \{nw } \\
\text { oumpion }\end{array}$ & $\underset{\substack{\text { ulp? } \\
\text { s!?ueno }}}{ }$ & $\begin{array}{c}\text { pason!jeo } \\
\text { чpreg }\end{array}$ & $\begin{array}{c}\text { Apegy } \\
\text { yozeg }\end{array}$ & $\begin{array}{l}\text { Luamiean } \\
\text {-add u!Beg }\end{array}$ & 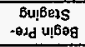 & $\begin{array}{c}\text { Aụjn!s } \\
\text { u!bog }\end{array}$ & Yque 1 Bu! bets & 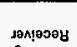 & $\begin{array}{c}(7 W) \\
\text { ownion }\end{array}$ & 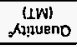 & Yxuet & yoreg & edopenug \\
\hline $\begin{array}{l}\text { volizez } \\
\text { of pasor }\end{array}$ & $\begin{array}{l}\text { qouww } \\
\text { go poos }\end{array}$ & 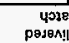 & Jad & 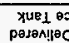 & $\begin{array}{l}\text { nos woy } \\
\text { paot lezoI }\end{array}$ & & & |W! $\mathbf{l}$ өleuțxo & & & $1458 M$ & Bu!jụnis & & 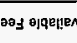 & & & \\
\hline
\end{tabular}

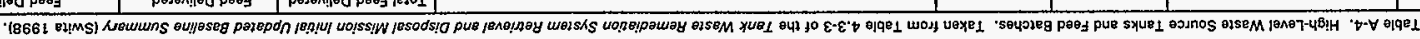


HNF-2168

Revision 0

\section{A.3 ANALYSIS OF THE TWRSO\&UP TRANSFER SCHEDULE}

Table A-5 presents the results of a computer-assisted analysis of the information in Table A-1. The "label" for each item is a designation assigned by the Hanford Site Technical Baseline Database (HSTBD) (Porter 1996); in some cases the description is slightly different than what is currently in the HSTBD to make it align better with the Double-Shell Tank System Specification (Grenard and Claghorn 1998); the batch volumes for an item reflect the quantities given in specific transfer records in Table A-1; the duration number is the end date of a specific transfer minus the start date plus one; the yearly volumes are summed for each type of transfer. In the event that a transfer spans the beginning of a new year (January 1) then a proportional quantity is assigned to each year. The total quantity is the sum from January 1, 1998, to January 1, 2011.

Table A-6 presents a map of the HSTBD designation for each type of transfer. A transfer type is defined by the "from" and "to" information in the transfer record. 
Table A-5. Analysis of TWRSO\&UP Transfers.

\begin{tabular}{|c|c|c|c|c|c|c|c|}
\hline \multirow[t]{2}{*}{ HSTBD number } & \multirow[t]{2}{*}{ Description } & \multicolumn{2}{|c|}{ Batch, gal } & \multicolumn{2}{|c|}{ Dur'n (d) } & \multirow{2}{*}{$\begin{array}{c}\text { Yearly } \\
\max , \text { gal }\end{array}$} & \multirow[t]{2}{*}{ Total, gal } \\
\hline & & Min & Max & Min & Max & & \\
\hline Water & Water & $5.00 \mathrm{E}+03$ & $5.59 \mathrm{E}+05$ & 1 & 6 & $2.25 \mathrm{E}+06$ & $1.30 \mathrm{E}+07$ \\
\hline tsd.1.2.5.1.F.11 & $\begin{array}{l}\text { Separated products (Entrained Solids, Sr-TRU, and } \\
\text { Technetium) from the Phase I treatment/immobilization } \\
\text { vendors returned to the DSTs. }\end{array}$ & $7.08 \mathrm{E}+03$ & $9.02 \mathrm{E}+03$ & 1 & 2 & $1.26 \mathrm{E}+05$ & $3.49 \mathrm{E}+05$ \\
\hline tsd.1.2.5.F.17 & Waste transferred to $242 \mathrm{~A}$ for evaporation. & $3.50 \mathrm{E}+04$ & $1.48 \mathrm{E}+06$ & 1 & 8 & $4.36 \mathrm{E}+06$ & $1.76 \mathrm{E}+07$ \\
\hline tsd.1.2.5.1.F.2 & Concentrated waste transferred to $\mathrm{AW}-106$. & $5.71 \mathrm{E}+02$ & $8.91 \mathrm{E}+05$ & 1 & 8 & $2.42 \mathrm{E}+06$ & $5.52 \mathrm{E}+06$ \\
\hline tsd.1.2.5.1.F.30 & $\begin{array}{l}\text { Liquid waste transferred cross site from West Area to East } \\
\text { Area. }\end{array}$ & $2.92 \mathrm{E}+04$ & $1.03 \mathrm{E}+06$ & 1 & 13 & $3.88 \mathrm{E}+06$ & $9.63 \mathrm{E}+06$ \\
\hline tsd.1.2.5.1.F.4 & $\begin{array}{l}\text { Waste transferred from one East Area DST to another East } \\
\text { Area DST. }\end{array}$ & $4.00 \mathrm{E}+03$ & $1.12 \mathrm{E}+06$ & 1 & 39 & $9.82 \mathrm{E}+06$ & $3.82 \mathrm{E}+07$ \\
\hline tsd.2.2.6.1.F.5 & $\begin{array}{l}\text { Mobilized/conditioned West Area DST waste prepared for } \\
\text { transfer for space management. }\end{array}$ & $2.77 \mathrm{E}+04$ & $1.08 \mathrm{E}+06$ & 1 & 6 & $5.05 \mathrm{E}+06$ & $5.05 \mathrm{E}+06$ \\
\hline tsd.2.2.6.F.6 & $\begin{array}{l}\text { LAW waste transferred to AP-102 or }-104 \text { for blending, } \\
\text { intermediate staging or qualification. }\end{array}$ & $2.80 \mathrm{E}+05$ & $1.01 \mathrm{E}+06$ & 2 & 6 & $5.77 \mathrm{E}+06$ & $2.09 \mathrm{E}+07$ \\
\hline di.wf.temp. 217 & $\begin{array}{l}\text { HLW transferred by } 222-S \text { Laboratory to the Double Shell } \\
\text { Tank (DST) System. }\end{array}$ & $6.00 \mathrm{E}+03$ & $3.70 \mathrm{E}+04$ & 1 & 1 & $6.20 \mathrm{E}+04$ & $3.43 E+05$ \\
\hline di.wf.temp.26.1 & $\begin{array}{l}\text { Liquid waste in East Area SSTs ready for transfer into West } \\
\text { Area DSTs including supernatant, interstitial liquids, and } \\
\text { separable organic wastes. }\end{array}$ & $0.00 \mathrm{E}+00$ & $0.00 \mathrm{E}+00$ & 0 & 0 & $0.00 \mathrm{E}+00$ & $0.00 \mathrm{E}+00$ \\
\hline di.wf.temp. 26.2 & $\begin{array}{l}\text { Liquid waste in West Area SSTS ready for transfer into West } \\
\text { Area DSTs including supernatant, interstitial liquids, and } \\
\text { separable organic wastes. }\end{array}$ & $1.11 \mathrm{E}+04$ & $1.50 \mathrm{E}+05$ & 7 & 83 & $6.00 \mathrm{E}+05$ & $1.20 \mathrm{E}+06$ \\
\hline isd.1.2.5.1.F.20 & $\begin{array}{l}\text { Supernatant from East Area DSTs for transfer to East Area } \\
\text { SST Sluicing Systems. }\end{array}$ & $9.21 \mathrm{E}+05$ & $9.21 \mathrm{E}+05$ & 5 & 5 & $9.21 E+05$ & $9.21 E+05$ \\
\hline tsd.1.2.5.1.F.8 & $\begin{array}{l}\text { Liquid waste from } 221-T \text { operations \& cleanup ready to be } \\
\text { transferred to West Area DSTs. }\end{array}$ & $1.00 \mathrm{E}+03$ & $5.40 \mathrm{E}+04$ & 1 & 1 & $5.60 \mathrm{E}+04$ & $3.09 \mathrm{E}+05$ \\
\hline isd.1,2,5,F.2 & HLW transferred from $105-\mathrm{F} \& 105-\mathrm{H}$ Basin. & $2.00 \mathrm{E}+04$ & $2.00 \mathrm{E}+05$ & 1 & 2 & $2.00 \mathrm{E}+05$ & $2.73 \mathrm{E}+05$ \\
\hline tsd.1.2.5.F.40 & $\begin{array}{l}\text { Liquid HLW transferred by Plutonium Finishing Plant. } \\
\text { Dates to be replanned based upon new funding profile. }\end{array}$ & $3.00 \mathrm{E}+03$ & $4.00 \mathrm{E}+03$ & 1 & 1 & $8.00 \mathrm{E}+03$ & $2.80 \mathrm{E}+04$ \\
\hline tsd.1.2.5.F.44 & HLW Transferred by Plutonium-Uranium Extraction Plant. & $5.00 \mathrm{E}+03$ & $1.00 \mathrm{E}+04$ & 1 & 1 & $1.00 \mathrm{E}+04$ & $1.50 \mathrm{E}+04$ \\
\hline tsd.1.2.5.F.49 & K Basin sludges transferred by $100 \mathrm{~K}$ Area Facilities. & $3.32 \mathrm{E}+05$ & $3.32 E+05$ & 2 & 2 & $3.32 \mathrm{E}+05$ & $3.32 \mathrm{E}+05$ \\
\hline tsd.1.2.5.F.69.1.3 & HLW transferred by WESF. & $5.00 \mathrm{E}+03$ & $1.00 \mathrm{E}+04$ & 1 & 1. & $1.00 \mathrm{E}+04$ & $6.00 \mathrm{E}+04$ \\
\hline tsd.1.2.5.F.69.2.2 & LLW (Liquid) Transferred by B Plant. & $4.40 \mathrm{E}+04$ & $4.40 \mathrm{E}+04$ & 1 & 1 & $4.40 \mathrm{E}+04$ & $4.40 \mathrm{E}+04$ \\
\hline tsd.1.2.5.F.7 & $\begin{array}{l}\text { Radioactive/mixed liquid waste transferred by } 340 \text { Waste } \\
\text { Handling Facility to DSTs. }\end{array}$ & $5.00 \mathrm{E}+04$ & $1.37 \mathrm{E}+05$ & 1 & 2 & $1.37 \mathrm{E}+05$ & $6.68 \mathrm{E}+05$ \\
\hline tsd.2.F.1 & $\begin{array}{l}\text { Waste retrieved via sluicing from East Area SSTs and } \\
\text { transferred to East Area DSTs. }\end{array}$ & $1.24 \mathrm{E}+03$ & $2.17 \mathrm{E}+06$ & 6 & 737 & $2.57 \mathrm{E}+06$ & $5.30 \mathrm{E}+06$ \\
\hline tsd.2.F.2 & $\begin{array}{l}\text { Waste retrieved via sluicing from West Area SSTs and } \\
\text { transferred to West Area DSTs. }\end{array}$ & $1.16 \mathrm{E}+04$ & $7.20 \mathrm{E}+05$ & 1 & 176 & $1.53 \mathrm{E}+06$ & $2.48 \mathrm{E}+06$ \\
\hline tsd.3.4.2.F.14.1 & $\begin{array}{l}\text { HLW Sludges retrieved from AZ-101 for transfer to the } \\
\text { Phase I, LAW/HLW Treatment/Immobilization Vendor. }\end{array}$ & $1.38 \mathrm{E}+05$ & $1.52 \mathrm{E}+05$ & 1 & 2 & $2.93 E+05$ & $1.57 \mathrm{E}+06$ \\
\hline tsd.3.4.2.F.9 & $\begin{array}{l}\text { LAW liquids and unintentionally entrained solids transferred } \\
\text { to the Phase I LAW treatment/immobilization vendor. }\end{array}$ & $1.71 \mathrm{E}+05$ & $1.01 \mathrm{E}+06$ & 2 & 7 & $3.21 \mathrm{E}+06$ & $1.37 \mathrm{E}+07$ \\
\hline isd.1.2.5.1.F.22 & $\begin{array}{l}\text { Liquid waste transferred from } 204-\mathrm{AR} \text { for storage in the East } \\
\text { Area DSTs. }\end{array}$ & $0.00 \mathrm{E}+00$ & $0.00 \mathrm{E}+00$ & 0 & 0 & $0.00 \mathrm{E}+00$ & $0.00 \mathrm{E}+00$ \\
\hline tsd.1.2.5.1.N.28W & Salt Well Pumping West & $8.30 \mathrm{E}+04$ & $4.35 E+05$ & 1 & 3 & $1.59 \mathrm{E}+06$ & $2.52 \mathrm{E}+0.6$ \\
\hline tsd.1.2.5.1.N.28E & Salt Well Pumping East & $1.86 \mathrm{E}+05$ & $1.86 \mathrm{E}+05$ & 2 & 2 & $1.86 \mathrm{E}+05$ & $1.86 \mathrm{E}+05$ \\
\hline tsd.1.2.5.1.C.28W & Salt Well Pumping West & $1.40 \mathrm{E}+04$ & $2.49 \mathrm{E}+05$ & 1 & 2 & $7.19 \mathrm{E}+05$ & $8.50 \mathrm{E}+05$ \\
\hline tsd.1.2.5.1.C.28E & Salt Well Pumping East & $1.41 \mathrm{E}+05$ & $7.75 \mathrm{E}+05$ & 2 & 4 & $7.75 \mathrm{E}+05$ & $9.16 \mathrm{E}+05$ \\
\hline
\end{tabular}


HNF-2168

Revision 0

Table A-6. Transfers Associated With Each Hanford Site Technical Database Designation.

\begin{tabular}{|c|c|c|c|}
\hline HSTBD number & Description & Transfer from & Transfer to \\
\hline$*$ tsd.1.2.5.1.F.11 & $\begin{array}{l}\text { Separated products (Entrained Solids, Sr-TRU, and } \\
\text { Technetium) from the Phase I treatment/immobilization } \\
\text { vendors returned to the DSTs. }\end{array}$ & $\begin{array}{l}\text { RETURN_1 } \\
\text { RETURN_2 }\end{array}$ & $\begin{array}{l}\text { AP-107 } \\
\text { AP-107 }\end{array}$ \\
\hline$*$ tsd.1,2,5.F.17 & Waste transferred to $242 \mathrm{~A}$ for evaporation & $A W-102$ & $242-\mathrm{A}$ \\
\hline$*$ tsd.1.2.5.1.F.2 & Concentrated waste transferred to AW-106. & $242-A$ & AW-106 \\
\hline$* t s d .1 .2 .5 .1 . F .22$ & $\begin{array}{l}\text { Liquid waste transferred from 204-AR for storage in the } \\
\text { East Area DSTs. }\end{array}$ & SPN87 & AN-105 \\
\hline$*$ tsd.1.2.5.1.F.28 & $\begin{array}{l}\text { Waste in West Area SSTs that has been transferred into } \\
\text { West Area DSTs. }\end{array}$ & $\begin{array}{l}\text { WNE88 } \\
\text { WCW88 } \\
\text { WNW88 } \\
\text { WCE88 } \\
\text { WNE88 } \\
\text { WCE88 }\end{array}$ & $\begin{array}{l}\text { SY-102 } \\
\text { SY-102 } \\
\text { SY-102 } \\
\text { AP-108 } \\
\text { AP-106 } \\
\text { AN-101 }\end{array}$ \\
\hline${ }^{*}$ tsd.1.2.5.1.F.30 & $\begin{array}{l}\text { Liquid waste transferred cross site from West Area to East } \\
\text { Area. }\end{array}$ & $\begin{array}{l}\text { SY-102 } \\
\text { SY-102 } \\
\text { SY-103 } \\
\text { SY-102 } \\
\text { SY-101 } \\
\text { SY-102 } \\
\text { SY-102 } \\
\text { SY-102 }\end{array}$ & $\begin{array}{l}\text { AW-102 } \\
\text { AN-107 } \\
\text { AN-102 } \\
\text { AP-104 } \\
\text { AN-102 } \\
\text { AN-102 } \\
\text { AW-105 } \\
\text { AP-107 }\end{array}$ \\
\hline
\end{tabular}


HNF-2168

Revision 0

Table A-6. Transfers Associated With Each Hanford Site Technical Database Designation.

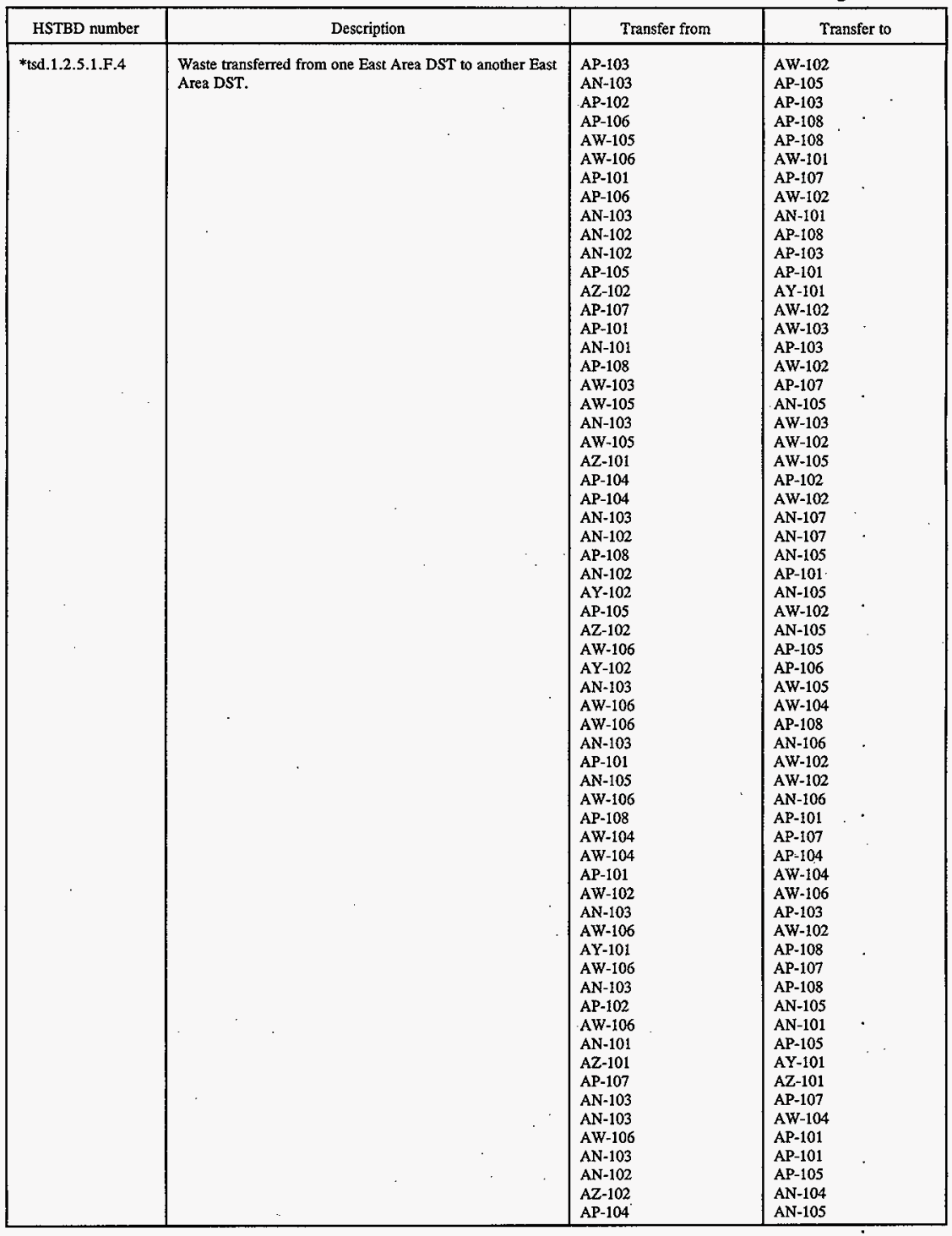


HNF-2168

Revision 0

Table A-6. Transfers Associated With Each Hanford Site Technical Database Designation.

\begin{tabular}{|c|c|c|c|}
\hline HSTBD number & Deseription & Transfer from & Transfer to \\
\hline *tsd.2.2.6.1.F.5 & $\begin{array}{l}\text { Mobilized/conditioned West Area DST waste prepared for } \\
\text { transfer for space management. }\end{array}$ & $\begin{array}{l}\text { SY-101 } \\
\text { SY-103 }\end{array}$ & $\begin{array}{l}S Y-102 \\
S Y-102\end{array}$ \\
\hline${ }^{*} \operatorname{tsd} .2 .2 .6 . F .6$ & $\begin{array}{l}\text { LAW waste transferred to AP-102 or }-104 \text { for blending, } \\
\text { intermediate staging or qualification. }\end{array}$ & $\begin{array}{l}\text { AN-105 } \\
\text { AW-101 } \\
\text { AN-107 } \\
\text { AN-107 } \\
\text { AN-106 } \\
\text { AN-104 } \\
\text { AW-101 } \\
\text { AY-101 } \\
\text { AN-103 } \\
\text { AN-105 } \\
\text { AN-102 } \\
\text { AN-104 } \\
\text { AN-103 } \\
\text { AN-106 } \\
\text { AY-101 } \\
\text { AN-102 }\end{array}$ & $\begin{array}{l}\text { AP-102 } \\
\text { AP-102 } \\
\text { AP-102 } \\
\text { AP-104 } \\
\text { AP-104 } \\
\text { AP-102 } \\
\text { AP-104 } \\
\text { AP-102 } \\
\text { AP-104 } \\
\text { AP-104 } \\
\text { AP-102 } \\
\text { AP-104 } \\
\text { AP-102 } \\
\text { AP-102 } \\
\text { AP-104 } \\
\text { AP-104 }\end{array}$ \\
\hline *D1.WF.temp. 217 & $\begin{array}{l}\text { HLW transferred by } 222-S \text { Laboratory to the Double Shell } \\
\text { Tank (DST) System. }\end{array}$ & $\begin{array}{l}\text { SPN87 } \\
\text { SPN87 }\end{array}$ & $\begin{array}{l}\text { SY-102 } \\
\text { AP-106 }\end{array}$ \\
\hline *DI.WF.temp.26.1 & $\begin{array}{l}\text { Liquid waste in East Area SSTs reàdy for transfer into } \\
\text { West Area DSTs including supernatant, interstitial liquids, } \\
\text { and separable organic wastes. }\end{array}$ & $\begin{array}{l}\text { B-111 } \\
\text { BX-106 } \\
\text { B-112 } \\
\text { BY-103 } \\
\text { BX-112 } \\
\text { B-111 } \\
\text { BX-102 } \\
\text { BY-110 } \\
\text { BY-110 } \\
\text { BX-112 } \\
\text { B-103 } \\
\text { BX-112 } \\
\text { BY-110 } \\
\text { BY-110 }\end{array}$ & $\begin{array}{l}\text { BA-6 } \\
\text { BA-4 } \\
\text { BA-1 } \\
\text { BA-5 } \\
\text { BA-2 } \\
\text { BA-1 } \\
\text { BA-2 } \\
\text { BA-3 } \\
\text { BA-6 } \\
\text { BA-3 } \\
\text { BA-1 } \\
\text { BA-4 } \\
\text { BA-5 } \\
\text { BA-4 }\end{array}$ \\
\hline . & $\begin{array}{l}\text { *DI.WF.temp. } 26.2 \text { Liquid waste in West Area SSTs } \\
\text { ready for transfer into West Area DSTs including } \\
\text { supermatant, interstitial liquids, and separable organic } \\
\text { wastes. }\end{array}$ & $\begin{array}{l}\text { U-106 } \\
\text { T-101 } \\
\text { U-202 } \\
\text { T-106 } \\
\text { U-101 } \\
\text { TY-101 } \\
\text { TY-101 } \\
\text { U-110 } \\
\text { U-104 } \\
\text { U-104 } \\
\text { TY-101 } \\
\text { TX-109 } \\
\text { TX-109 } \\
\text { U-106 } \\
\text { U-112 } \\
\text { TX-109 } \\
\text { U-112 } \\
\text { U-203 } \\
\text { U-201 } \\
\text { U-110 } \\
\text { T-101 } \\
\text { U-204 } \\
\text { TY-105 } \\
\text { TX-109 } \\
\text { U-111 }\end{array}$ & $\begin{array}{l}\text { UA-2 } \\
\text { TA-1 } \\
\text { UA-1 } \\
\text { TA-1 } \\
\text { UA-1 } \\
\text { TA-3 } \\
\text { TA-5 } \\
\text { UA-1 } \\
\text { UA-1 } \\
\text { UA-2 } \\
\text { TA-2 } \\
\text { TA-2 } \\
\text { TA-1 } \\
\text { UA-1 } \\
\text { UA-1. } \\
\text { TA-6 } \\
\text { UA-2 } \\
\text { UA-1 } \\
\text { UA-1 } \\
\text { UA-2 } \\
\text { TA-3 } \\
\text { UA-1 } \\
\text { TA-2 } \\
\text { TA-4 } \\
\text { UA-2 }\end{array}$ \\
\hline
\end{tabular}


Table A-6. Transfers Associated With Each Hanford Site Technical Database Designation.

\begin{tabular}{|c|c|c|c|c|}
\hline HSTBD number & Description & Transfer from & \multicolumn{2}{|c|}{ Transfer to } \\
\hline${ }^{*}$ tsd.1,2.5.1.F.20 & $\begin{array}{l}\text { Supernatant from East Area DSTs for transfer to East Area } \\
\text { SST Sluicing Systems. }\end{array}$ & AY-102 & $\mathrm{C}-106$ & \\
\hline$*$ tsd.1.2.5.1.F.8 & $\begin{array}{l}\text { Liquid waste from } 221-T \text { operations \& cleanup ready to be } \\
\text { transferred to West Area DSTs. }\end{array}$ & $\begin{array}{l}\text { TNS88 } \\
\text { TAL88 } \\
\text { TNS88 } \\
\text { TAL88 }\end{array}$ & $\begin{array}{l}\text { AP-106 } \\
\text { AN-105 } \\
\text { AN-105 } \\
\text { AP-106 }\end{array}$ & · \\
\hline$*_{\text {tsd.1.2.5.F.2 }}$ & HLW transferred from 105-F \& 105-H Basin. & $\begin{array}{l}\text { 1NS96 } \\
1 \text { FL96 }\end{array}$ & $\begin{array}{l}\text { AW-105 } \\
\text { AW-105 }\end{array}$ & \\
\hline$*$ tsd.1.2.5.F.40 & $\begin{array}{l}\text { Liquid HLW transferred by Plutonium Finishing Plant. } \\
\text { Dates to be replanned based upon new funding profile. }\end{array}$ & ZNL87 & SY-102 & \\
\hline$*_{\text {tsd.1 }}$ 2.5.F.44 & $\begin{array}{l}\text { HLW Transferred by Plutonium-Uranium Extraction } \\
\text { Plant. }\end{array}$ & $\begin{array}{l}\text { PXTCO } \\
\text { PXTCO }\end{array}$ & $\begin{array}{l}A W-105 \\
A P-106\end{array}$ & \\
\hline${ }^{*}$ tsd.1.2.5.F.49 & $\mathrm{K}$ Basin sludges transferred by $100 \mathrm{~K}$ Area Facilities. & $1 \mathrm{KL} 96$ & AW-105 & \\
\hline *tsd.1.2.5.F.69.1.3 & HLW transferred by WESF. & $\begin{array}{l}\text { WESF } \\
\text { WESF }\end{array}$ & $\begin{array}{l}\text { AP-106 } \\
\text { AN-105 }\end{array}$ & . \\
\hline$* t s d .1 .2 .5 . F .69 .2 .2$ & LLW (Liquid) Transferred by B Plant. & $\begin{array}{l}\text { BPTCO } \\
\text { BPTCO }\end{array}$ & $\begin{array}{l}\text { AP-106 } \\
\text { AP-108 }\end{array}$ & \\
\hline$*$ tsd.1.2.5.F.7 & $\begin{array}{l}\text { Radioactive/mixed liquid waste transferred by } 340 \text { Waste } \\
\text { Handling Facility to DSTs. }\end{array}$ & $\begin{array}{l}34 \mathrm{~L} 87 \\
34 \mathrm{~L} 87\end{array}$ & $\begin{array}{l}\text { AP-106 } \\
\text { AN-105 }\end{array}$ & \\
\hline$*$ tsd.2.F.1 & - & $\begin{array}{l}\text { C-106 } \\
\text { AX-104 } \\
\text { AX-103 } \\
\text { A-102 } \\
\text { A-106 } \\
\text { A-105 } \\
\text { C-103 } \\
\text { AX-101 } \\
\text { C-202 } \\
\text { AX-102 } \\
\text { C-201 } \\
\text { C-111 } \\
\text { C-204 } \\
\text { A-103 } \\
\text { C-108 } \\
\text { C-105 } \\
\text { A-104 } \\
\text { C-203 } \\
\text { BA-3 } \\
\text { BA-2 } \\
\text { BA-2 } \\
\text { BA-6 } \\
\text { BA-3 } \\
\text { BA-1 } \\
\text { BA-5 } \\
\text { BA-6 } \\
\text { BA-4 } \\
\text { BA-5 } \\
\text { BA-1 } \\
\text { BA-3 } \\
\text { BA-4 } \\
\text { BA-2 }\end{array}$ & $\begin{array}{l}\text { AY-102 } \\
\text { AN-103 } \\
\text { AN-103 } \\
\text { AN-103 } \\
\text { AN-103 } \\
\text { AN-103 } \\
\text { AN-103 } \\
\text { AN-103 } \\
\text { AN-103 } \\
\text { AN-103 } \\
\text { AN-103 } \\
\text { AN-103 } \\
\text { AN-103 } \\
\text { AN-103 } \\
\text { AN-103 } \\
\text { AN-103 } \\
\text { AN-103 } \\
\text { AN-103 } \\
\text { AP-102 } \\
\text { AP-102 } \\
\text { AP-105 } \\
\text { AP-105 } \\
\text { AP-103 } \\
\text { AP-102 } \\
\text { AP-106 } \\
\text { AP-108 } \\
\text { AP-106 } \\
\text { AP-101 } \\
\text { AP-101 } \\
\text { AP-101 } \\
\text { AP-101 } \\
\text { AP-101 }\end{array}$ & . \\
\hline
\end{tabular}


Table A-6. Transfers Associated With Each Hanford Site Technical Database Designation.

\begin{tabular}{|c|c|c|c|}
\hline HSTBD number & Description & Transfer from & Transfer to \\
\hline${ }^{*}$ tsd.2.F.2 & $\begin{array}{l}\text { Waste retrieved via sluicing from West Area SSTs and } \\
\text { transferred to West Area DSTs. }\end{array}$ & $\begin{array}{l}\text { SX-110 } \\
\text { S-105 } \\
\text { SX-113 } \\
\text { SX-115 } \\
\text { SX-106 } \\
\text { S-103 } \\
\text { S-102 } \\
\text { SX-112 } \\
\text { SX-111 } \\
\text { TA-4 } \\
\text { TA-1 } \\
\text { TA-2 } \\
\text { TA-6 } \\
\text { TA-3 } \\
\text { UA-1 } \\
\text { TA-5 } \\
\text { UA-2 }\end{array}$ & $\begin{array}{l}\text { SY-103 } \\
\text { SY-101 } \\
\text { SY-103 } \\
\text { SY-103 } \\
\text { SY-103 } \\
\text { SY-101 } \\
\text { SY-101 } \\
\text { SY-103 } \\
\text { SY-103 } \\
\text { SY-102 } \\
\text { SY-102 } \\
\text { SY-102 } \\
\text { SY-102 } \\
\text { SY-102 } \\
\text { SY-102 } \\
\text { SY-102 } \\
\text { SY-102 }\end{array}$ \\
\hline$*$ tsd.3.4.2.F.14.1 & $\begin{array}{l}\text { HLW Sludges retrieved from AZ-101 for transfer to the } \\
\text { Phase I, LAW/HLW Treatment/Immobilization Vendor. }\end{array}$ & $\begin{array}{l}\text { AZ-101 } \\
\text { AY-102 } \\
\text { AZ-102 }\end{array}$ & $\begin{array}{l}\text { P1HLW_FEED_TANK } \\
\text { P1HLW_FEED_TANK } \\
\text { P1HLW_FEED_TANK }\end{array}$ \\
\hline$*_{\mathrm{tsd}}, 3,4,2, \mathrm{~F}, 9$ & $\begin{array}{l}\text { LAW liquids and unintentionally entrained solids } \\
\text { transferred to the Phase I LAW treatment/immobilization } \\
\text { vendor. }\end{array}$ & AP-102 & $\begin{array}{l}\text { AP-106 } \\
\text { AP-108 }\end{array}$ \\
\hline *Water & . & $\begin{array}{l}\text { WATER } \\
\text { WATER } \\
\text { WATER } \\
\text { EVAPF } \\
\text { LERF } \\
\text { WATER } \\
\text { WASH-WATER } \\
\text { WATER } \\
\text { LERF } \\
\text { EVAPF } \\
\text { LERF } \\
\text { EVAPF } \\
\text { LERF } \\
\text { WATER } \\
\text { WATER } \\
\text { WATER } \\
\text { WASH-WATER } \\
\text { EVAPF } \\
\text { WASH-CAUSTIC } \\
\text { WATER } \\
\text { WASH-WATER } \\
\text { LERF } \\
\text { EVAPF } \\
\text { LERF } \\
\text { WATER } \\
\text { WASH-WATER } \\
\text { WATER } \\
\text { LERF } \\
\text { WASH-WATER } \\
\text { LERF } \\
\text { LERF }\end{array}$ & $\begin{array}{l}\text { SY-102 } \\
\text { AW-105 } \\
\text { AP-108 } \\
\text { SY-102 } \\
\text { AN-102-PUMP } \\
\text { AW-102 } \\
\text { AY-102 } \\
\text { AP-106 } \\
\text { AN-106-PUMP } \\
\text { AW-102 } \\
\text { AN-103-PUMP } \\
\text { AP-106 } \\
\text { SY-103-PUMP } \\
\text { AN-101 } \\
\text { AP-105 } \\
\text { AP-107 } \\
\text { AZ-101 } \\
\text { AN-105 } \\
\text { AN-107 } \\
\text { AP-104 } \\
\text { AZ-102 } \\
\text { AW-101-PUMP } \\
\text { AW-106 } \\
\text { AN-104-PUMP } \\
\text { AN-105 } \\
\text { AP-102 } \\
\text { AY-102 } \\
\text { AN-105-PUMP } \\
\text { AP-104 } \\
\text { SY-101-PUMP } \\
\text { AN-107-PUMP }\end{array}$ \\
\hline
\end{tabular}


HNF-2168

Revision 0

\section{A.4 REQUIREMENT SPECIFIC DERIVATIONS}

Table A-7 provides additional detail regarding the derivation of each of the performance requirements. The paragraph number refers to the paragraph of the DST System Specification (Grenard and Claghorn 1998). 
Table A-7. Requirement Specific Derivations. (4 Sheets)

\begin{tabular}{|c|c|c|c|c|c|c|c|c|}
\hline \multirow[t]{2}{*}{$\begin{array}{l}\text { Req } \\
\text { No }\end{array}$} & \multirow[t]{2}{*}{ Paragraph } & \multirow[t]{2}{*}{ Title } & \multirow[t]{2}{*}{ HSTBD number } & \multirow[t]{2}{*}{ HSTBD description } & \multicolumn{2}{|c|}{$\begin{array}{c}\text { Quantity associated } \\
\text { with HSTBD number }\end{array}$} & \multirow[t]{2}{*}{ Units } & \multirow[t]{2}{*}{ Reference table, location } \\
\hline & & & & & Min & $\operatorname{Max}$ & & \\
\hline 1 & $3.2 .1 .2 .2 . \mathrm{a}$ & $\begin{array}{l}200 \text { West Area Waste Batch. } \\
\text { Transfer }\end{array}$ & tsd.2.2.6.1.F.5 & & $2.77+04$ & $1.08+06$ & gal & $\begin{array}{l}\text { A-5 (Min \& Max Batch Size) } \\
\text { See Table A-8 for duration }\end{array}$ \\
\hline 2 & $3.2 .1 .2 .5 . \mathrm{a}$ & $\begin{array}{l}200 \text { East Area Waste Batch } \\
\text { Transfer }\end{array}$ & tsd.1.2.5.1.F.4 & & $4.00+03$ & $1.12+06$ & gal & A-5 (Min \& Max Batch Size) \\
\hline 3 & $3.2 .1 .2 .3 . \mathrm{a}$ & $\begin{array}{l}\text { Cross-site Waste Transfer } \\
\text { Volume }\end{array}$ & tsd.1.2.5.1.F.30 & & $2.92+04$ & $1.03+06$ & gal & A-5 (Min \& Max Batch Size) \\
\hline 4 & $3.2 .1 .4 .3 . a$ & $\begin{array}{l}\text { High-Level Waste Batch } \\
\text { Sizes, Phase I }\end{array}$ & tsd.3.4.2.2.F.3 & . & $4.76+04$ & $2.91+05$ & gal & A-4 (Min \& Max Available Feed) \\
\hline \multirow[t]{3}{*}{5} & \multirow[t]{3}{*}{ 3.2.1.4.3.b } & \multirow{3}{*}{$\begin{array}{l}\text { High-Level Waste Preparation } \\
\text { Phase I }\end{array}$} & \multirow[t]{2}{*}{ tsd.3.4.2.2.F.3 } & Add transfer solution & 1 & 2. & days & A-10 (Min. duration) \\
\hline & & & & Qualify HLW & 315 & 315 & & A-10 (Min. Duration) \\
\hline & & & Total duration & & 316 & 317 & & \\
\hline 6 & $3.2 .1 .4 .4 . a$ & $\begin{array}{l}\text { High-Level Waste Batch } \\
\text { Volume }\end{array}$ & tsd.3.4.2.F.14.1 & & $7.13+04$ & $1.45+0.5$ & gal & A-4 (Min \& Max Batch Delivered) \\
\hline 7 & 3.2.1.2.1.c & $\begin{array}{l}\text { Insoluble Waste Preparation - } \\
\text { West Area Tanks }\end{array}$ & tsd.2.2.6.1.F.5 & & 1 & 14 & days & See Table 2 \\
\hline 8 & 3.2.1.2.4.c & $\begin{array}{l}\text { Insoluble Waste Preparation - } \\
\text { East Area Tanks }\end{array}$ & tsd.2.2.6.1.F.6 & & 1. & 13 & days & A-10 (Min. Transfer Time) \\
\hline 9 & 3.2.1.2.7.a & $\begin{array}{l}\text { Low-Activity Waste Staging } \\
\text { Batch Volumes }\end{array}$ & tsd.2.2.6.F.6 & & $2.80+05$ & $1.01+06$ & gal & A-5 (Min \& Max Batch Size) \\
\hline . 10 & 3.2.1.2.a & $\begin{array}{l}\text { Low-Activity Waste Staging } \\
\text { Quantity, Phase I }\end{array}$ & tsd.2.2.6.F.6 & & & 10,500 & MT & A-3 (Total Feed Delivered) \\
\hline \multirow[t]{2}{*}{11} & \multirow[t]{2}{*}{ 3.2.1.3.1.a } & \multirow[t]{2}{*}{$\begin{array}{l}\text { Low-Activity Waste Staging } \\
\text { Batch Size, Phase I }\end{array}$} & \multirow[t]{2}{*}{ tsd.2.2.6.F.6 } & \multirow[t]{2}{*}{ 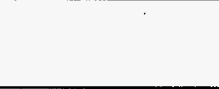 } & $2.80+05$ & $1.01+06$ & gal & $\begin{array}{l}\text { A-5 (Min \& Max Batch Size) } \\
\text { A-3 (Min \& Max Batch Size) }\end{array}$ \\
\hline & & & & & 113 & 120 & days & A-11 (Min. and Max Duration) \\
\hline 12 & 3.2.1.3.2.a & $\begin{array}{l}\text { Low-Activity Waste Batches, } \\
\text { Phase I }\end{array}$ & tsd.2.2.6.F.6 & & $1.96+05$ & $1.01+06$ & gal & A-3 (Min \& Max Batch Size) \\
\hline 13 & 3.2.1.4.2.a & $\begin{array}{l}\text { Sludge Wash Supernatant } \\
\text { Total Volume }\end{array}$ & tsd.1.2.5.F.57 & & & $2.30+06$ & gal & A-12 (Transfer Volume) \\
\hline 14 & 3.2.1.4.2.b & $\begin{array}{l}\text { Sludge Wash Supernatant } \\
\text { Batch Volume }\end{array}$ & tsd.1.2.5.F.57 & & $6.61+04$ & $3.70+05$ & gal & $\begin{array}{l}\text { A-12 (Min. and Max Transfer } \\
\text { Volume) }\end{array}$ \\
\hline 15 & 3.2.1.2.1.b & $\begin{array}{l}\text { Soluble Waste Preparation - } \\
\text { West Area }\end{array}$ & tsd.2.2.6.1.F.5 & & 82 & 118 & days & A-15 (Up to First Decant) \\
\hline 16 & $3.2 .1 .2 .4 . \mathrm{b}$ & $\begin{array}{l}\text { Soluble Waste Preparation - } \\
\text { East Area }\end{array}$ & tsd.2.2.6.1.F.7 & & 10 & 117 & days & A-13 (Up to Second Set of Decants) \\
\hline
\end{tabular}


Table A-7. Requirement Specific Derivations. (4 Sheets)

\begin{tabular}{|c|c|c|c|c|c|c|c|c|}
\hline \multirow[t]{2}{*}{$\begin{array}{l}\text { Req } \\
\text { No }\end{array}$} & \multirow[t]{2}{*}{ Paragraph } & \multirow[t]{2}{*}{ Title } & \multirow[t]{2}{*}{ HSTBD number } & \multirow[t]{2}{*}{ HSTBD description } & \multicolumn{2}{|c|}{$\begin{array}{c}\text { Quantity associated } \\
\text { with HSTBD number }\end{array}$} & \multirow[t]{2}{*}{ Units } & \multirow[t]{2}{*}{ Reference table, location } \\
\hline & & & & & Min & $\operatorname{Max}$ & & \\
\hline \multirow[t]{5}{*}{17} & \multirow[t]{5}{*}{$3.2 .1 .2 . \mathrm{b}$} & \multirow{5}{*}{$\begin{array}{l}\text { Space Management Annual } \\
\text { Waste Volume, Phase I }\end{array}$} & tsd.1.2.5.1.F.4 & East DST to East DST & & $9.82 \mathrm{E}+06$ & $\mathrm{gal} / \mathrm{yr}$ & A-5 (Yearly max) \\
\hline & & & tsd.1.2.5.F.17 & AW-102 to Evaporator & & $4.36 \mathrm{E}+06$ & $\mathrm{gal} / \mathrm{yr}$ & A-5 (Yeariy max) \\
\hline & & & tsd.2.2.6.1.F.5 & West DST to West DST & & $5.05 \mathrm{E}+06$ & $\mathrm{gal} / \mathrm{yr}$ & A-5 (Yearly max) \\
\hline & & & tsd.1.2.5.1.R.30 & West DST to East DST & & $3.88 \mathrm{E}+06$ & $\mathrm{gal} / \mathrm{yr}$ & A-5 (Yearly max) \\
\hline & & & Total & & & $2.31 \mathrm{E}+07$ & gailyr & \\
\hline 18 & 3.2.1.2.1.a & $\begin{array}{l}\text { Supernatant Preparation - } \\
\text { West Area }\end{array}$ & tsd.2.2.6.1.R.5 & & NA & NA & & See Table 2 \\
\hline 19 & $3.2 .1 .2 .4 . a$ & $\begin{array}{l}\text { Supernatant Preparation - East } \\
\text { Area }\end{array}$ & tsd.2.2.6.1.F.7 & & NA & NA & & See Table 2 \\
\hline 20 & 3.2.3.10.a & Water - Phase I & Water & Water & & $1.30 \mathrm{E}+07$ & $\mathrm{gal}$ & A-5 (Total) \\
\hline 21 & $3.2 .1 .4 . d$ & $\begin{array}{l}\text { High-Level Waste Feed } \\
\text { Frequency, Phase I }\end{array}$ & tsd.3.4.2.F.14.1 & & 200 & 295 & days & A-4 (Process Time) \\
\hline 22 & 3.2.1.3.d & $\begin{array}{l}\text { Low-Activity Waste Feed } \\
\text { Rrequency, Phase I }\end{array}$ & tsd.3.4.2.F.9 & & 125 & 182 & days & A-11 (Total Duration) \\
\hline 23 & 3.2.1.1.8.a & $\begin{array}{l}\text { Treatment Plant Waste } \\
\text { Product Volume, Phase I }\end{array}$ & tsd.1.2.5.1.F.11 & AP-107 Returns & & $1.26 \mathrm{E}+05 \mid$ & $\mathrm{gal} / \mathrm{yr}$ & A-5 (Yearly Max) \\
\hline \multirow[t]{4}{*}{24} & \multirow[t]{4}{*}{ 3.2.1.1.3.a } & \multirow[t]{4}{*}{ 204-AR Annual Capacity } & ssd.1.2.5.F.2 & 100 Area & & $2.00 \mathrm{E}+05$ & $\mathrm{gal} / \mathrm{yr}_{\mathrm{r}}$ & A-5 (Yearly max) \\
\hline & & & tsd.1.2.5.F.7 & 300 Area & & $1.37 \mathrm{E}+05$ & $\mathrm{gal} / \mathrm{yr}_{\mathrm{r}}$ & A-5 (Yearly max) \\
\hline & & & tsd.1.2.5.1.F.8 & T-Plant & & $5.60 \mathrm{E}+04$ & $\mathrm{gal} / \mathrm{yr}$ & A-5 (Yearly max) \\
\hline & & & Total & & & $3.93 \mathrm{E}+05$ & $\mathrm{gal} / \mathrm{yr}$ & \\
\hline 25 & $3.2 .1 .1 .3 . b$ & $\begin{array}{l}\text { 204-AR Batch Transfer } \\
\text { Capability }\end{array}$ & tsd.1.2.5.1.F.22 & & & 5,000 & gal & See Table 2 \\
\hline 26 & $3.2 .1 .1 .6 . \mathrm{a}$ & $\begin{array}{l}\text { Annual Volume of } \\
\text { Concentrated Waste }\end{array}$ & tsd.1.2.5.1.F.2 & & & $2.42 \mathrm{E}+06$ & $\mathrm{gal} / \mathrm{yr}$ & A-5 (Yearly Max) \\
\hline \multirow[t]{3}{*}{27} & \multirow[t]{3}{*}{$3.2 .3 .4 . a$} & \multirow{3}{*}{$\begin{array}{l}\text { B Plant/Waste Encapsulation } \\
\text { and Storage Pacility Waste } \\
\text { Volume }\end{array}$} & tsd.1.2.5.F.69.1.3 & WESF & & $1.00 \mathrm{E}+04$ & $\mathrm{gal} / \mathrm{yr}$ & A-5 (Yearly Max) \\
\hline & & & tsd.1.2.5.F.69.2.2 & B Plant & & $4.40 \mathrm{E}+04$ & $\mathrm{gal} / \mathrm{yr}$ & A-5 (Yearly max) \\
\hline & & & Total & & & $5.40 \mathrm{E}+04$ & $\mathrm{gal} / \mathrm{yr}$ & \\
\hline 28 & 3.2.3.6.a & $\begin{array}{l}\text { Concentrated Waste Received } \\
\text { from the Evaporator }\end{array}$ & tsd.1.2.5.1.F.2 & & & $8.91 \mathrm{E}+05$ & gal & A-5 (Max Batch Size) \\
\hline \multirow[t]{5}{*}{29} & \multirow[t]{5}{*}{$3.2 .1 .1 .5 . \mathrm{a}$} & \multirow{5}{*}{$\begin{array}{l}\text { East Area Liquid Waste } \\
\text { Annual Capacity }\end{array}$} & tsd.1.2.5.F. 2 & 100 Area & & $2.00 \mathrm{E}+05$ & $\mathrm{gal} / \mathrm{yr}$ & A-5 (Yearly Max) \\
\hline & & & Esd.1.2.5.F.44 & PUREX & & $1.00 \mathrm{E}+04$ & $\mathrm{gal} / \mathrm{yr}$ & A-5 (Yearly Max) \\
\hline & & & tsd.1.2.5.P.69.1.3 & WESF & & $1.00 \mathrm{E}+04$ & $\mathrm{gal} / \mathrm{yr}$ & A-5 (Yearly Max) \\
\hline & & & tsd.1.2.5.F.69.2.2 & B Plant & & $4.40 \mathrm{E}+04$ & $\mathrm{gal} / \mathrm{yr}$ & A-5 (Yearly Max) \\
\hline & & & tssd.1.2.5.P.7 & 300 Area & & $1.37 \mathrm{E}+05$ & $\mathrm{gal} / \mathrm{yr}$ & A-5 (Yearly Max) \\
\hline
\end{tabular}


Table A-7. Requirement Specific Derivations. (4 Sheets)

\begin{tabular}{|c|c|c|c|c|c|c|c|c|}
\hline \multirow[t]{6}{*}{$\begin{array}{l}\text { Req } \\
\text { No }\end{array}$} & \multirow[t]{6}{*}{ Paragraph } & \multirow[t]{6}{*}{ Title } & \multirow{3}{*}{\begin{tabular}{|l} 
HSTBD number \\
tsd.2.P.1. \\
\end{tabular}} & \multirow[t]{2}{*}{ HSTBD description } & \multicolumn{2}{|c|}{$\begin{array}{c}\text { Quantity associated } \\
\text { with HSTBD number }\end{array}$} & \multirow[t]{2}{*}{ Units } & \multirow[t]{2}{*}{ Reference table, location } \\
\hline & & & & & Min & $\operatorname{Max}$ & & \\
\hline & & & & SST Retrieval & & $2.57 \mathrm{E}+06$ & $\mathrm{gal} / \mathrm{yr}$ & A-5 (Yearly Max) \\
\hline & & & tsd.1.2.5.1.N:28E & Salt well DN & & $1.86 \mathrm{E}+05$ & $\mathrm{gal} / \mathrm{yr}$ & A-5 (Yearly Max) \\
\hline & & & tsd.1.2.5.1.C.28E & Salt Well DC & & $7.75 \mathrm{E}+05$ & $\mathrm{gal} / \mathrm{yr}$ & A-5 (Yearly Max) \\
\hline & & & Total & & & $3.94 \mathrm{E}+06$ & $\mathrm{gal} / \mathrm{yr}_{\mathrm{r}}$ & A-5 (Yearly Max) \\
\hline \multirow[t]{3}{*}{30} & \multirow[t]{3}{*}{$3.2 .3 .5 . b$} & \multirow{3}{*}{$\begin{array}{l}\text { East Single-Shell Tank Salt } \\
\text { Well Liquid Waste Volume }\end{array}$} & tsd.1.2.5.1.N.28E & Salt Well DN & & $1.86 \mathrm{E}+05$ & gal & A-5 (Total) \\
\hline & & & tsd.1.2.5.1.C.28E & Salt Well DC & & $9.16 \mathrm{E}+05$ & gal & A-5 (Total) \\
\hline & & & Total & & & $1.10 \mathrm{E}+06$ & & \\
\hline 31 & $3.2 .1 .2 .6 . a$ & $\begin{array}{l}\text { Evaporator Feed Annual } \\
\text { Volume }\end{array}$ & tsd.1.2.5.P.17 & & & $4.36 \mathrm{E}+06$ & $\mathrm{gal} / \mathrm{yr}$ & A-5 (Yearly Max) \\
\hline 32 & $3.2 .1 .2 .6 . \mathrm{b}$ & $\begin{array}{l}\text { Evaporator Feed Max Batch } \\
\text { Size }\end{array}$ & tsd.1.2.5.F.17 & & $3.50 \mathrm{E}+04$ & $1.48 \mathrm{E}+06$ & gal & A-5 (Min \& Max Batch Size) \\
\hline 33 & $3.2 .1 .2 . \mathrm{c}$ & $\begin{array}{l}\text { Evaporator Feed Volume, } \\
\text { Phase I }\end{array}$ & tsd.1.2.5.F.17 & & & $4.36 \mathrm{~B}+06$ & $\mathrm{gal} / \mathrm{yr}$ & A-5 (Yearly Max) \\
\hline \multirow[t]{7}{*}{34} & \multirow[t]{7}{*}{3.2 .1 .1 .4 .6} & \multirow[t]{7}{*}{ Waste Batch Size - West Area } & di.wf.temp. 217 & $222-\mathrm{S} \mathrm{Lab}$ & $6.00 \mathrm{E}+03$ & $3.70 \mathrm{E}+04$ & gal & A-5 (Min \& Max Batch Size) \\
\hline & & & tsd.2.F.2 & SST Retrieval & $1.16 \mathrm{E}+04$ & $7.20 \mathrm{E}+05$ & gal & A-5 (Min \& Max Batch Size) \\
\hline & & & tsd.1.2.5.1.F.8 & T-Plant & $1.00 \mathrm{E}+03$ & $5.40 \mathrm{E}+04$ & gal & A-5 (Min \& Max Batch Size) \\
\hline & & & tsd.1.2.5.F.40 & PFP & $3.00 \mathrm{E}+03$ & $4.00 \mathrm{E}+03$ & gal & A-5 (Min \& Max Batch Size) \\
\hline & & & tsd.1.2.5.1.C.28W & Sait Well DN & $1.40 \mathrm{E}+04$ & $2.49 \mathrm{E}+05$ & gal & A-5 (Min \& Max Batch Size) \\
\hline & & & tsd.1.2.5.1.N.28W & Salt Well DC & $8.30 \mathrm{E}+04$ & $4.35 \mathrm{E}+05$ & gal & A-5 (Min \& Max Batch Size) \\
\hline & & & Bounds & & $1.00 \mathrm{E}+03$ & $7.20 \mathrm{E}+05$ & gal & \\
\hline \multirow[t]{8}{*}{35} & \multirow[t]{8}{*}{3.2 .1 .1 .5 .6} & \multirow{8}{*}{$\begin{array}{l}\text { Liquid Waste Batch Size - } \\
\text { East Area }\end{array}$} & tsd.1.2.5.R.2 & 100 Area & $2.00 \mathrm{E}+04$ & $2.00 \mathrm{E}+05$ & gal & A-5 (Min \& Max Batch Size) \\
\hline & & & tsd.1.2.5.F.44 & PUREX & $5.00 \mathrm{E}+03$ & $1.00 \mathrm{E}+04$ & gal & A-5 (Min \& Max Batch Size) \\
\hline & & & tsd.1.2.5.F.69.1.3 & WESF & $5.00 \mathrm{E}+03$ & $1.00 \mathrm{E}+04$ & gal & A-5 (Min \& Max Batch Size) \\
\hline & & & tsd.1.2.5.F.69.2.2 & B Plant & $4.40 \mathrm{E}+04$ & $4.40 \mathrm{E}+04$ & gal & A-5 (Min \& Max Batch Size) \\
\hline & & & $\operatorname{tsd}$ 1.2.5.F.7 & 300 Area & $5.00 \mathrm{E}+04$ & $1.37 \mathrm{E}+05$ & gal & A-S (Min \& Max Batch Size) \\
\hline & & & tsd.1.2.5.1.N.28E & Salt Well DN & $1.86 \mathrm{E}+05$ & $1.86 \mathrm{E}+05$ & gal & A-5 (Min \& Max Batch Size) \\
\hline & & & tsd.1.2.5.1.C.28E & Sait Well DC & $1.41 \mathrm{E}+05$ & $7.75 \mathrm{E}+05$ & gal & A-5 (Min \& Max Batch Size) \\
\hline & & & Bounds & & $5.00 \mathrm{E}+03$ & $7.75 \mathrm{E}+05$ & gal & \\
\hline 36 & 3.2.3.2.a & $\begin{array}{l}\text { Plutonium Finishing Plant } \\
\text { Waste Annual Volume } \\
\end{array}$ & tsd.1.2.5.F.40 & PFP & & $8.00 \mathrm{E}+03$ & $\mathrm{gal} / \mathrm{yr}$ & A-5 (Yearly Max) \\
\hline 37 & 3.2.3.3.a & S Plant (222-S) Waste Volume & di.wf.temp.217 & $(222-S)$ & & $6.20 \mathrm{E}+04$ & $\mathrm{gal} / \mathrm{yr}$ & A-5 (Yearly Max) \\
\hline \multirow[t]{2}{*}{38} & \multirow[t]{2}{*}{ 3.2.3.5.d } & \multirow{2}{*}{$\begin{array}{l}\text { Verify Single-Shell Tank } \\
\text { Waste Retrieval Volume, } \\
\text { Phase I I }\end{array}$} & tsd.2.F. 1 & East & & $5.30 \mathrm{E}+06$ & gal & A-5 (Total) \\
\hline & & & tssd.2.F.2 & West & & $2.48 \mathrm{E}+06$ & gal & A-5 (Total) \\
\hline
\end{tabular}


Table A-7. Requirement Specific Derivations. (4 Sheets)

\begin{tabular}{|c|c|c|c|c|c|c|c|c|}
\hline \multirow[t]{3}{*}{$\begin{array}{l}\text { Req } \\
\text { No }\end{array}$} & \multirow[t]{3}{*}{ Paragraph } & \multirow[t]{3}{*}{ Title } & \multirow[t]{2}{*}{ HSTBD number } & \multirow[t]{2}{*}{ HSTBD description } & \multicolumn{2}{|c|}{$\begin{array}{l}\text { Quantity associated } \\
\text { with HSTBD number }\end{array}$} & \multirow[t]{2}{*}{ Units } & \multirow[t]{2}{*}{ Reference table, location } \\
\hline & & & & & Min & $\operatorname{Max}$ & & \\
\hline & & & Total & 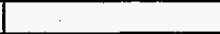 & & $7.79 \mathrm{E}+06$ & gal & \\
\hline \multirow[t]{7}{*}{39} & \multirow[t]{7}{*}{$3.2 .1 .1 .4 . \mathrm{a}$} & \multirow{7}{*}{$\begin{array}{l}\text { West Area Liquid Waste } \\
\text { Annual Capacity }\end{array}$} & di.wf.temp.217 & 222-S Lab & & $6.20 \mathrm{E}+04$ & $\mathrm{gal} / \mathrm{yr}$ & A-5 (Ýearly Max) \\
\hline & & & tsd.2.F.2 & SST Retrieval & & $1.53 \mathrm{E}+06$ & $\mathrm{gal} / \mathrm{yr}$ & A-5 (Yearly Max) \\
\hline & & & tsd.1.2.5.1.F.8 & T-Plant & & $5.60 \mathrm{E}+04$ & $\mathrm{gal} / \mathrm{yr}$ & A-5 (Yearly Max) \\
\hline & & & tsd.1.2.5.F.40 & PFP & & $8.00 \mathrm{E}+03$ & $\mathrm{gal} / \mathrm{yr}$ & A-5 (Yearly Max) \\
\hline & & & tsd.1.2.5.1.N.28W & Salt Well DN & & $1.59 \mathrm{E}+06$ & $\mathrm{gal} / \mathrm{yr}$ & A-5 (Yearly Max) \\
\hline & & & tsd.1.2.5.1.C.28W & Salt Well DC & & $7.19 E+05$ & $\mathrm{gal} / \mathrm{yr}$ & A-5 (Yearly Max) \\
\hline & & & Total & & & $3.96 \mathrm{E}+06$ & $\mathrm{gal} / \mathrm{yr}$ & \\
\hline \multirow[t]{3}{*}{40} & \multirow[t]{3}{*}{$3.2 .3 .5 . \mathrm{a}$} & \multirow{3}{*}{$\begin{array}{l}\text { Verify West Single-Shell Tank } \\
\text { Salt Well Liquid Waste } \\
\text { Volume }\end{array}$} & isd.1.2.5.1.N.28W & Salt Well DN & & $2.52 \mathrm{E}+06$ & gal & A-5 (Total) \\
\hline & & & tsd.1.2.5.1.C. $28 \mathrm{~W}$ & Salt Well DC & & $8.50 \mathrm{E}+05$ & $\mathrm{gal}$ & A-5 (Total) \\
\hline & & & Total & & & $3.37 \mathrm{E}+06$ & gal & \\
\hline
\end{tabular}


Table A-8. Cost Estimating Input Sheet.

S6FF

116 Perform Transfer from AP-102 to AP-106

15.8. 150.844

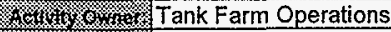

w w o w 6 . IT Bohan $N$ Mongar/M Lee

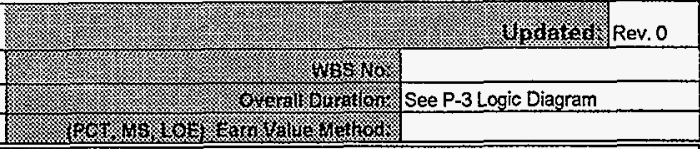

' 00,000 gallons of waste from AP-102 to the Vendor's tank AP-106. This transfer will use a transfer previousiy prepared in 130.B40.F1 saving approximately 4 weeks off the schedule. No mixing ir settling is required prior to the transfer. Work performed in this activity includes prerequisites, transfer, and post transfer operations. Specific activities included in the prerequisites include Jperator (NPO) review of the procedures, perform valve line-up and cover block checks with support from the Operating Engineer (1st line supervisor) and the Shift Manager. Transfer ops inciude ransfer pump startup and instrument monitoring in the operations control building during the transfer. Post transfer ops include operator debriefing, Shift Manager/Operating Engineer reviews, the ZOG Engineer review, and the QA record closeout of the transfer.

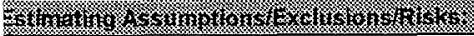

W-314 delivers upgrade to master pump shutdown CKT which allows less field surveillance during transfer (currently takes up to 8 NPOs to do this).

No transfer equipment failures that add to cost, transfer EQ is maintained operable as base operations.

3. W-211 delivers transfer equipment to support transfer toffrom AP-102/4/6/8.

1. No mixing or settling is required prior to transfer.

AP-102 and AP-104 cannot be done in parallel.

5. Tank AP-106 is flushed and empty. 7. Transfer procedure previously prepared in 130.B40.F1 can be used for this transfer.

Ti.

Jurrent practices, $100+$ transfers per year.

1) $700,000 \mathrm{gal} / 80 \mathrm{gpm}(7000 / 1440) \times 1.25$ efficiency $=7.59$ days SAY 8 days

i. 2 NPO's $\times 2$ shifts $\times 12$ hrs $\times 8$ days $=384$

$1 \mathrm{OE} \times 2$ shifts $\times 12$ hrs $\times 8$ days $=192$

?. Schedule duration allows for 2 -days to perform prerequisites (review documentation, routing, valve lineups, etc.), 8-days to perform the actual transfer base on volume divided gallons per minute umping capacity, and finally \{-day to do post ops transfer activities (re-align valves, debriefings, internal reporting, etc.)

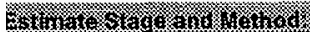

t team of subject matter experts consisting of Management, Engineering/Operations staff were initially interviewed for an understanding of the activity scope and work process. Assumptions made in expected throughput and technical requirements are assumed or relatively unknowm. Parametrics based on planning \& process methodology unit rates for this work were used as the basis. THE ISTIMATE FOR THIS ACTIVITY IS CONSIDERED PRELIMINARY

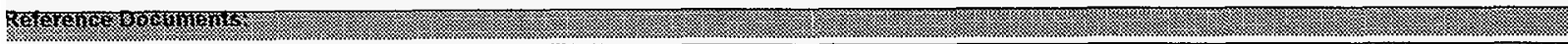
See Technical Basis Review document, section 2 "Reference Documents and Reports." 
Table A-8. Cost Estimating Input Sheet.

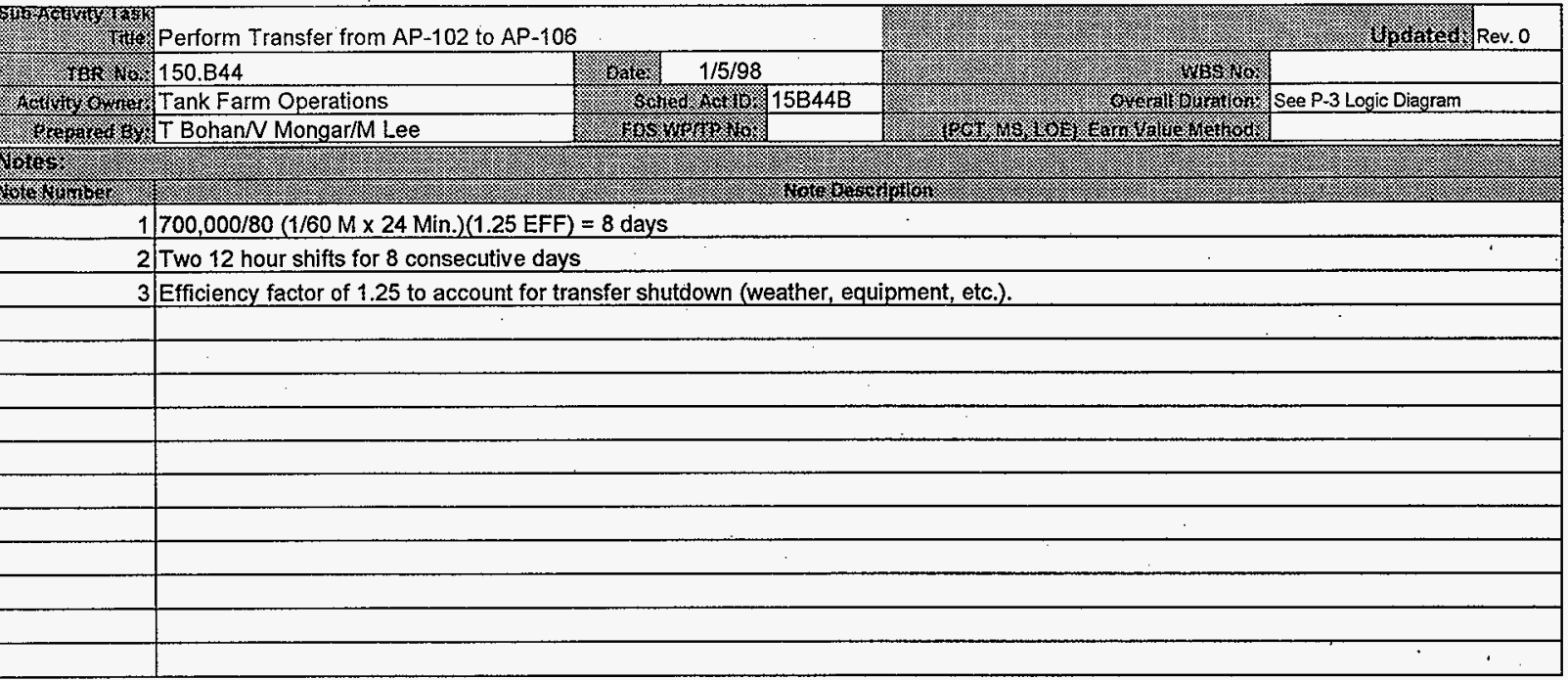


Table A-9. Pretreatment Processing Durations. (Taken from Table 3.2-3 of the TWRSO\&UP)

\begin{tabular}{|c|c|c|c|c|c|}
\hline \multirow{2}{*}{ Operation } & \multirow{2}{*}{$\begin{array}{l}\text { Minimum } \\
\text { duration } \\
\text { per } \\
\text { repetition }\end{array}$} & \multirow{2}{*}{$\begin{array}{l}\text { Maximum } \\
\text { duration } \\
\text { per } \\
\text { repetition }\end{array}$} & \multicolumn{3}{|c|}{ Repetitions } \\
\hline & & & 241-AZ-101 & 241-AZ-102 & $\begin{array}{c}241-\mathrm{AY}-102 / \\
241-\mathrm{C}-106\end{array}$ \\
\hline $\begin{array}{l}\text { Decant Initial } \\
\text { Supernatant }^{\mathrm{a}}\end{array}$ & $1-2$ days & 30 days & 1 & 1 & 1 \\
\hline $\begin{array}{l}\text { Add wash } \\
\text { solution }^{\mathrm{a}}\end{array}$ & $1-2$ days & \multirow[t]{3}{*}{$\begin{array}{c}30 \text { days } \\
\text { total }\end{array}$} & 3 & 4 & 2 \\
\hline $\begin{array}{l}\text { Mix waste with } \\
\text { mixer pumps }\end{array}$ & $\begin{array}{c}5 \text { days } \\
\text { (estimated) }\end{array}$ & & 3 & 4 & 2 \\
\hline 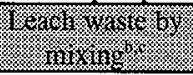 & 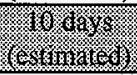 & & (3) & 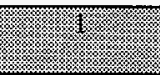 & . \\
\hline $\begin{array}{c}\text { Allow waste to } \\
\text { settle }\end{array}$ & 30 days & 30 days & 3 & 4 & 2 \\
\hline $\begin{array}{c}\text { Sample and } \\
\text { analyze solids } \\
\text { and } \\
\text { supernatant }^{\mathrm{f}}\end{array}$ & 14 days & \multirow[t]{2}{*}{$\begin{array}{c}30 \text { days } \\
\text { total }\end{array}$} & 3 & 4 & 2 \\
\hline $\begin{array}{c}\text { Decant } \\
\text { supernatant/ } \\
\text { wash solution }^{\mathrm{a}}\end{array}$ & $1-2$ days & & 3 & 4 & 2 \\
\hline $\begin{array}{l}\text { Minimum total } \\
\text { time }^{\mathrm{d}}\end{array}$ & & & 161 days & $\begin{array}{l}214 \text { days } \\
\text { Max }\end{array}$ & $\begin{array}{l}108 \text { days } \\
\text { Min }\end{array}$ \\
\hline $\begin{array}{c}\text { Total allocated } \\
\text { time } \\
\text { (maximum } \\
\text { total time) }\end{array}$ & & & 300 days & 390 days & 210 days \\
\hline
\end{tabular}

${ }^{2}$ The minimum durations for waste transfers are based on a $0.73 \mathrm{ML} /$ day pump rate. The maximum durations for waste transfers are a conservatively high estimate to account for equipment failures, transfer conflicts, etc. (Certa et al. 1996).

${ }^{b}$ Actual mixing time will vary depending upon the results of the $\mathrm{W}-151$ mixer pump test.

'This step is performed only if caustic leaching is required, and is not used for the "Base Case" schedule.

${ }^{\mathrm{d}}$ The total minimum and maximum times include the initial decant step.

'The total allocated time should be used for planning purposes.

${ }^{\mathrm{f}}$ This sampling and analysis required here is to verify the progress of the pretreatment process for a few critical waste components (e.g., aluminum, sulfate, sodium). 
HNF-2168

Revision 0

Table A-10. Feed Staging Durations for Tanks 241-AZ-101, 241-AZ-102, and 241-AY-102/241-C-106. (Taken from Table 3.2-4 of the TWRSO\&UP)

\begin{tabular}{|c|c|c|}
\hline Operation & $\begin{array}{c}\text { Minimum } \\
\text { duration }\end{array}$ & Allocated time \\
\hline Add transfer solution ${ }^{\mathrm{a}}$ & $1-2$ days & 10 days \\
\hline $\begin{array}{c}\text { Mix/sample/evaluate feed source, } \\
\text { qualify waste form }\end{array}$ & $\begin{array}{c}315 \text { days } \\
\text { (estimated) }\end{array}$ & 315 days \\
\hline $\begin{array}{c}\text { Transfer high-level waste batch to } \\
\text { Privatization Contractor }^{\mathrm{a}}\end{array}$ & 1 day & 14 days \\
\hline Total $^{\mathrm{b}}$ & 318 days & 339 days \\
\hline
\end{tabular}

${ }^{a} B a s e d$ on a $0.73 \mathrm{ML} /$ day pump rate.

'See Section 3.2.1.1 for discussion on how pretreatment and feed staging durations combine to determine a total duration. 
Table A-11. Feed Delivery Cycle Times.

\begin{tabular}{|c|c|c|}
\hline \multicolumn{2}{|l|}{ Batch } & \multirow{2}{*}{$\left.\begin{array}{c}\text { Duration (days) } \\
182 \\
69 \\
28 \\
85\end{array}\right\}_{113}$} \\
\hline $\begin{array}{l}\text { Batch 1, Envelope A } \\
\text { Mobilize and Retrieve LAW from Source Tank } \\
\text { Adjust Staged Feed As Required } \\
\text { Feed Qualification }\end{array}$ & TOTAL & \\
\hline $\begin{array}{l}\text { Batch 2, Envelope A } \\
\text { Mobilize and Retrieve LAW from Source Tank } \\
\text { Adjust Staged Feed As Required } \\
\text { Feed Qualification }\end{array}$ & TOTAL & $\left.\begin{array}{c}182 \\
69 \\
28 \\
85\end{array}\right\}$ \\
\hline $\begin{array}{l}\text { Batch 3, Envelope A } \\
\text { Mobilize and Retrieve LAW from Source Tank } \\
\text { Adjust Staged Feed As Required . } \\
\text { Feed Qualification }\end{array}$ & TOTAL & $\left.\begin{array}{c}182 \\
69 \\
28 \\
85\end{array}\right\} 113$ \\
\hline $\begin{array}{l}\text { Batch 4, Envelope A } \\
\text { Mobilize and Retrieve LAW from Source Tank } \\
\text { Adjust Staged Feed As Required } \\
\text { Feed Qualification }\end{array}$ & TOTAL & $\left.\begin{array}{r}182 \\
69 \\
28 \\
85\end{array}\right\} 113$ \\
\hline $\begin{array}{l}\text { Batch 5, Envelope A } \\
\text { Retrieve LAW from Source Tanks } \\
\text { Adjust Staged Feed As Required } \\
\text { Feed Qualification }\end{array}$ & TOTAL & $\left.\begin{array}{c}142 \\
22 \\
35 \\
85\end{array}\right\} 120$ \\
\hline $\begin{array}{l}\text { Batch 6, Envelope B } \\
\text { Retrieve LAW from Source Tank } \\
\text { Adjust Staged Feed As Required } \\
\text { Feed Qualification }\end{array}$ & TOTAL & $\left.\begin{array}{l}123 \\
10 \\
28 \\
85\end{array}\right\}_{113}$ \\
\hline $\begin{array}{ll}\text { Batch } 7 / 8, \text { Envelope C } \\
& \text { Retrieve LAW from Source Tank } \\
\text { Note: } & \text { this shortened duration requires feed qualification in th } \\
& \text { retrieval with mass balance correction for dilution in th } \\
\text { basis for payment. }\end{array}$ & $\begin{array}{l}\text { TOTAL } \\
\text { waste } \\
\text { tablish }\end{array}$ & $\begin{array}{l}\mathbf{1 0} \\
10\end{array}$ \\
\hline $\begin{array}{l}\text { Batch 9, Envelope C } \\
\text { Mobilize and Retrieve LAW from Source Tank } \\
\text { Adjust Staged Feed As Required } \\
\text { Feed Qualification }\end{array}$ & TOTAL & $\left.\begin{array}{l}125 \\
12 \\
28 \\
85\end{array}\right\}_{113}$ \\
\hline $\begin{array}{l}\text { Batch 10, Envelope C } \\
\text { Mobilize and Retrieve LAW from Source Tank } \\
\text { Adjust Staged Feed As Required } \\
\text { Feed Qualification }\end{array}$ & TOTAL & $\left.\begin{array}{l}125 \\
12 \\
28 \\
85\end{array}\right\} 113$ \\
\hline $\begin{array}{l}\text { Batch 11, Envelope } C \\
\text { Mobilize and Retrieve LAW from Intermediate Tank } \\
\text { Adjust Staged Feed As Required } \\
\text { Feed Qualification }\end{array}$ & TOTAL & $\left.\begin{array}{r}122 \\
9 \\
28 \\
85\end{array}\right\}$ \\
\hline $\begin{array}{l}\text { Batch 12, Envelope C } \\
\text { Mobilize and Retrieve LAW from Intermediate Tank } \\
\text { Adjust Staged Feed As Required } \\
\text { Feed Qualification }\end{array}$ & TOTAL & $\left.\begin{array}{c}122 \\
9 \\
28 \\
85\end{array}\right\}$ \\
\hline
\end{tabular}

Note: This information extracted from the supporting documentation for the Tank Waste Remediation System Retrieval and Disposal Mission Initial Updated Baseline Summary (Swita 1998). 
HNF-2168

Revision 0

Table A-12. Schedule for Phase I High-Level Waste Pretreatment and Feed Staging. (Sheet 1 of 3) (Taken from Table 3.2-5 of the TWRSO\&UP)

\begin{tabular}{|c|c|c|c|}
\hline Activity description & $\begin{array}{c}\text { Transfer } \\
\text { volume }(L)\end{array}$ & Late start date & $\begin{array}{l}\text { Duration } \\
\text { (days) }\end{array}$ \\
\hline \multicolumn{4}{|c|}{ "Bootstrap" Transfer Supporting AZ-101 Pretreatment } \\
\hline 1. Transfer AY-101 Supernatant to AP-106 & $3,200,000$ & $8 / 30 / 00$ & 30 \\
\hline \multicolumn{4}{|c|}{ Other Operations Supporting AZ-101 Pretreatment } \\
\hline ** Perform AZ-101 Mixer Pump Test & & $4 / 21 / 98$ & 30 \\
\hline ** Obtain Grab Samples of Sludge from AZ-101 for PHMC & & $5 / 21 / 98$ & 14 \\
\hline 2a. Complete AZ-101 In-Tank Evaporation to $5 \mathrm{M} \mathrm{Na}$ & 0 & $8 / 30 / 00$ & 0 \\
\hline \multicolumn{4}{|c|}{ "Bootstrap" Transfer Supporting AZ-101 Pretreatment } \\
\hline 3. Transfer AZ-101 Supernatant to AY-101 & $2,400,000$ & $8 / 30 / 00$ & 30 \\
\hline \multicolumn{4}{|c|}{ AZ-101 Siudge Washing Operations } \\
\hline 4a. Add Wash Solution to AZ-101 and Mix Sludge & 550,000 & $9 / 29 / 00$ & 30 \\
\hline 5a. Wait for Sludge to Settle in AZ-101 & & $10 / 29 / 00$ & 30 \\
\hline 6a. Sample Sludge and Decant AZ-101 Supernatant to AW-105 & 600,000 & $11 / 28 / 00$ & 30 \\
\hline 4b. Add Wash Solution to AZ-101 and Mix Sludge & 550,000 & $12 / 28 / 00$ & 30 \\
\hline 5b. Wait for Sludge to Settle in AZ-101 & & $1 / 27 / 01$ & 30 \\
\hline 6b. Sample Sludge and Decant AZ-101 Supernatant to AW-105 & 520,000 & $2 / 26 / 01$ & 30 \\
\hline 4c. Add Wash Solution to AZ-101 and Mix Sludge & 550,000 & $3 / 28 / 01$ & 30 \\
\hline 5c. Wait for Sludge to Settle in AZ-101 & & $4 / 27 / 01$ & 30 \\
\hline 6c. Sample Sludge and Decant AZ-101 Supernatant to AW-105 & 540,000 & $5 / 27 / 01$ & 30 \\
\hline \multicolumn{4}{|c|}{ AZ-101 Feed Staging Operations } \\
\hline 7. Add Transfer Solution to AZ-101 & 550,000 & $6 / 26 / 01$ & 10 \\
\hline ** Mix/Sample/Evaluate AZ-101 Sludge & & $7 / 6 / 01$ & 70 \\
\hline $\begin{array}{l}\text { ** Take Representative Samples from AZ-101, Provide Them to } \\
\text { the Privatization Contractor, and Wait for Waste Form } \\
\text { Qualification }\end{array}$ & & $9 / 14 / 01$ & 245 \\
\hline 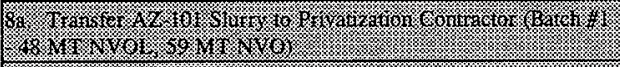 & 3 & 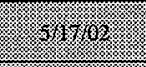 & 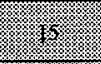 \\
\hline 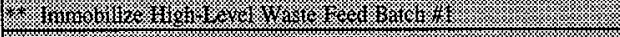 & & 6 & \% \\
\hline 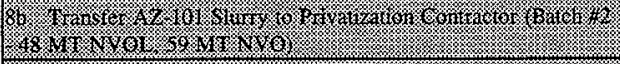 & 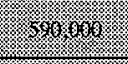 & 3.6. & 缕 \\
\hline 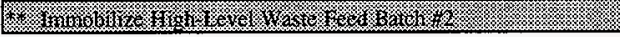 & 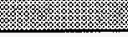 & 8 & 20 \\
\hline
\end{tabular}


Table A-12. Schedule for Phase I High-Level Waste Pretreatment and Feed Staging. (Sheet 2 of 3) (Taken from Table 3.2-5 of the TWRSO\&UP)

\begin{tabular}{|c|c|c|c|}
\hline Activity description & $\begin{array}{c}\text { Transfer } \\
\text { volume (L) } \\
\end{array}$ & Late start date & $\begin{array}{l}\text { Duration } \\
\text { (days) }\end{array}$ \\
\hline \multicolumn{4}{|c|}{ Other Operations Supporting AZ-102 Pretreatment } \\
\hline ** Obtain Core Samples from AZ-102 for PHMC & 4 & TBD & 150 \\
\hline 2b. Complete AZ-102 In-Tank Evaporation to $5 \mathrm{MNa}$ & $1,700,000$ & $1 / 8 / 02$ & 0 \\
\hline \multicolumn{4}{|c|}{ "Bootstrap" Transfer Supporting AZ-102 Pretreatment } \\
\hline 9. Transfer AZ-102 Supernatant to AY-101 and AN-104 & $1,300,000$ & $1 / 8 / 02$ & 30 \\
\hline \multicolumn{4}{|c|}{ AZ-102 Sludge Washing Operations } \\
\hline 10a. Add Wash Solution to AZ-102 and Mix Sludge & 810,000 & $2 / 7 / 02$ & 30 \\
\hline 11a. Wait for Sludge to Settle in AZ-102 & 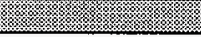 & $3 / 9 / 02$ & 30 \\
\hline $\begin{array}{l}\text { 12a. Sample Sludge and Decant AZ-102 Supernatant to AN- } \\
105\end{array}$ & $\begin{array}{c}250,000 \\
\text { Min }\end{array}$ & $4 / 8 / 02$ & 30 \\
\hline 10b. Add Wash Solution to AZ-102 and Mix Sludge & 810,000 & $5 / 8 / 02$ & 30 \\
\hline 11b. Wait for Sludge to Settle in AZ-102 & \$ & $6 / 7 / 02$ & 30 \\
\hline $\begin{array}{l}\text { 12b. Sample Sludge and Decant AZ-102 Supernatant to AN- } \\
105\end{array}$ & 780,000 & $7 / 7 / 02$ & 30 \\
\hline 10c. Add Wash Solution to AZ-102 and Mix Sludge & 810,000 & $8 / 6 / 02$ & 30 \\
\hline 11c. Wait for Sludge to Settle in AZ-102 & & $9 / 5 / 02$ & 30 \\
\hline $\begin{array}{l}\text { 12c. Sample Sludge and Decant AZ-102 Supernatant to AN- } \\
105\end{array}$ & 790,000 & $10 / 5 / 02$ & 30 \\
\hline 10d. Add Wash Solution to AZ-102 and Mix Sludge & 810,000 & $11 / 4 / 02$ & 30 \\
\hline 11d. Wait for Sludge to Settle in AZ-102 & & $12 / 4 / 02$ & 30 \\
\hline $\begin{array}{l}\text { 12d. Sample Sludge and Decant AZ-102 Supernatant to AN- } \\
105\end{array}$ & 800,000 & $1 / 3 / 03$ & 30 \\
\hline \multicolumn{4}{|c|}{ AZ-102 Feed Staging Operations } \\
\hline 13. Add Transfer Solution to $\mathrm{AZ}-102$ & 820,000 & $2 / 2 / 03$ & 10 \\
\hline ** Mix/Sample/Evaluate AZ-102 Sludge & & $2 / 12 / 03$ & 70 \\
\hline $\begin{array}{l}\text { ** Take Representative Samples from AZ-102, Provide Them } \\
\text { to the Privatization Contractor, and Wait for Waste Form } \\
\text { Qualification }\end{array}$ & & $4 / 23 / 03$ & 245 \\
\hline W. & & 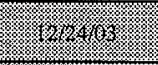 & \\
\hline 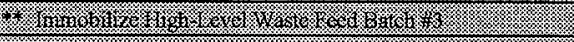 & & 傮 & res \\
\hline W. & & 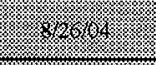 & (3) \\
\hline 样 & & 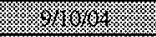 & 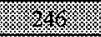 \\
\hline 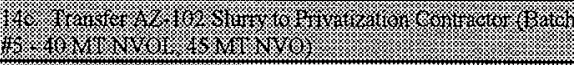 & & 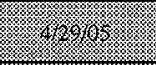 & (x) \\
\hline (6) & & 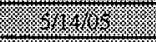 & 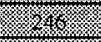 \\
\hline W & & & 格 \\
\hline 4. & (3) & 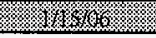 & 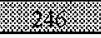 \\
\hline
\end{tabular}


Table A-12. Schedule for Phase I High-Level Waste Pretreatment and Feed Staging. (Sheet 3 of 3) (Taken from Table 3.2-5 of the TWRSO\&UP)

\begin{tabular}{|c|c|c|c|}
\hline Activity description & $\begin{array}{c}\text { Transfer } \\
\text { volume (L) }\end{array}$ & Late start date & $\begin{array}{c}\text { Duration } \\
\text { (days) }\end{array}$ \\
\hline \multicolumn{4}{|c|}{ Other Operations Supporting AY-102 Pretreatment } \\
\hline ** Obtain Core Samples from AY-102 for PHMC & r) & TBD & 150 \\
\hline \multicolumn{4}{|c|}{ AY-102 Sludge Washing Operations } \\
\hline 15. Sample Sludge and Decant AY-102 Supernatant to AN-105 & $2,000,000$ & $3 / 16 / 05$ & 30 \\
\hline 16a. Add Wash Solution to AY-102 and Mix Sludge & $1,200,000$ & $4 / 15 / 05$ & 30 \\
\hline 17a. Wait for Sludge to Settle in AY-102 & & $5 / 15 / 05$ & 30 \\
\hline 18a. Sample Sludge and Decant AY-102 Supernatant to AN-105 & $1,400,000 \mathrm{Max}$ & $6 / 14 / 05$ & 30 \\
\hline 16b. Add Wash Solution to AY-102 and Mix Sludge & $1,200,000$ & $7 / 14 / 05$ & 30 \\
\hline 17b. Wait for Sludge to Settle in AY-102 & & $8 / 13 / 05$ & 30 \\
\hline 18b. Sample Sludge and Decant AY-102 Supernatant to AN-105 & $1,200,000$ & $9 / 12 / 05$ & 30 \\
\hline \multicolumn{4}{|c|}{ AY-102 Feed Staging Operations } \\
\hline 19. Add Transfer Solution to AY-102 & 1,60 & $10 / 12 / 05$ & 10 \\
\hline ** Mix/Sample/Evaluate AY-102 Sludge & & $10 / 22 / 05$ & 70 \\
\hline $\begin{array}{l}\text { ** Take Representative Samples from AY-102, Provide Them to } \\
\text { the Privatization Contractor, and Wait for Waste Form } \\
\text { Qualification }\end{array}$ & & $12 / 31 / 05$ & 245 \\
\hline (20, & & & \\
\hline 洋, & & . & 33 \\
\hline (1) & & & \\
\hline 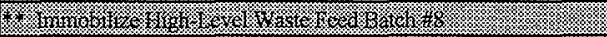 & & 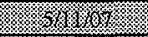 & $\frac{1}{3}$ \\
\hline (6) & (3) & $2 \%$ & 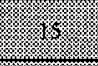 \\
\hline 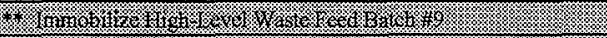 & 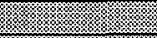 & $\sqrt{2}$ & 4 \\
\hline W & & & \\
\hline W & & 8 8. X 64 & 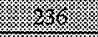 \\
\hline (2018 & 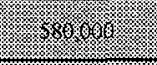 & (1) & 学 \\
\hline 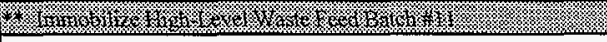 & - & 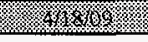 & 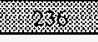 \\
\hline $\begin{array}{l}\text { ** Complete High-Level Waste Immobilization (450 MT N } \\
590 \text { MT NVO) }\end{array}$ & & $12 / 10 / 09$ & 0 \\
\hline
\end{tabular}

NVOL $=$ Non-Volatile Oxides Excluding Sodium and Silicon

General Notes:

NVO $=$ Non-Volatile Oxides

- The activity numbers correspond to the illustration in Figure 3.4-11.

- Activities with no numbers (**) are not represented in Figure 3.4-11.

- The waste transfers shown in this schedule are based on conservative maximum durations, and may not correspond to the modeled schedule provided in Appendix $\mathrm{H}$. 


\section{LAW Preparation and Staging Times}

Table A-13. Soluble Waste Preparation - East Area Tanks with Saltcake (AN-103, AN-104, AN-105, AW-101).

\begin{tabular}{|c|c|l|}
\hline Calendar days & $\begin{array}{c}\text { P3 schedule } \\
\text { activity ID }\end{array}$ & \multicolumn{1}{|c|}{ Activity title } \\
\hline 60 & 13B25B & Degas AN-105 \\
\hline 2 & $13 \mathrm{~B} 30 \mathrm{~A}$ & Add Diluent ( 25 KGal) \\
\hline 6 & $13 \mathrm{~B} 30 \mathrm{~B} 2$ & Decant AN-105 to AP-102 \\
\hline 6 & $13 \mathrm{~B} 30 \mathrm{C} 2$ & Decant AN-105 to AP-104 \\
\hline 5 & $13 \mathrm{~B} 30 \mathrm{D}$ & Add Diluent Water to AN-105 \\
\hline 5 & $13 \mathrm{~B} 30 \mathrm{E} 1$ & Operate Mixer Pumps in AN-105 \\
\hline 3 & $13 \mathrm{~B} 30 \mathrm{~F}$ & Perform Grab Sampling in AN-105 \\
\hline 30 & $13 \mathrm{~B} 30 \mathrm{G}$ & Settle Solids in AN-105 \\
\hline 8 & $13 \mathrm{~B} 30 \mathrm{H}$ & Decant waste from AN-105 to AP-102 \\
\hline 8 & $13 \mathrm{~B} 30 \mathrm{~J}$ & Decant waste from AN-105 to AP-104 \\
\hline 133 & TOTAL & \\
\hline
\end{tabular}

Table A-14. Soluble Waste Preparation - East Area Tanks with Supernatant Retrieval Only (AY-101, AN-107, AN-102, AN-106).

\begin{tabular}{|c|c|l|}
\hline Calendar days & $\begin{array}{c}\text { P3 schedule } \\
\text { activity ID }\end{array}$ & \multicolumn{1}{|c|}{ Activity title } \\
\hline 10 & $13 \mathrm{M} 30 \mathrm{~B}$ & Transfer $800 \mathrm{KGal}$ from AN-106 to AP-102 \\
\hline 10 & $13 \mathrm{M} 40 \mathrm{~B}$ & Transfer $800 \mathrm{KGal}$ from AN-106 to AP-104 \\
\hline 20 & TOTAL & \\
\hline
\end{tabular}


Table A-15. Soluble Waste Preparation - West Area Tanks (SY-101, SY-103).

\begin{tabular}{|c|c|l|}
\hline Calendar days & $\begin{array}{c}\text { P3 schedule } \\
\text { activity ID }\end{array}$ & \multicolumn{1}{|c|}{ Activity title } \\
\hline 60 & 13R10B & Degas SY-101 \\
\hline 4 & 13R30A & Add Diluent ( 32 KGal) \\
\hline 8 & 13R30C & Decant 538 KGal from SY-101 to SY-102 \\
\hline 8 & 13R30D & Add Diluent 500 KGal to SY-101 \\
\hline 5 & 13R30E & Mix SY-101 \\
\hline 3 & 13R30F & Perform Grab Sampling in SY-101 (12MAR06- \\
\hline 30 & 13R30G & Settle SY-101 \\
\hline 8 & 13R30H & Decant SY-101 to SY-102 500 KGal \\
\hline 5 & 13R35B & Mix 102-SY \\
\hline 15 & 13R35C & Transfer 800 KGal from SY-102 to AN-102 \\
\hline 9 & 13R40A & Decant 500 KGal from SY-101 to SY-102 \\
\hline 5 & 13R50B & Mix 102-SY \\
\hline 15 & 13R50C & Transfer 800 KGal from SY-102 to AN-107 \\
\hline 10 & 13R45B & Transfer 800 KGal from AN-102 to AP-102 \\
\hline 10 & 13R55B & Transfer 800 KGal from AN-107 to AP-104 \\
\hline 195 & TOTAL & \\
\hline & & \\
\hline
\end{tabular}


The Blend/Qualify and Deliver Times for Staged LAW are the same for all batches except Batch 7 (AN-107). Batch 7 is qualified in the source tank prior to transfer to AP-102/AP-104.

Table A-16. Blend and Qualify Staged Low-Activity Waste.

\begin{tabular}{|c|c|l|}
\hline Calendar days & $\begin{array}{c}\text { P3 schedule } \\
\text { activity ID }\end{array}$ & \multicolumn{1}{|c|}{ Activity title } \\
\hline 5 & $15 \mathrm{~B} 42 \mathrm{~B}$ & Perform AP-102 Mixing Operation \\
\hline 13 & $15 \mathrm{~B} 42 \mathrm{C1}$ & $\begin{array}{l}\text { Obtain and Analyze Process Control Samples in AP- } \\
102\end{array}$ \\
\hline 7 & $15 \mathrm{~B} 42 \mathrm{E} 1$ & $\begin{array}{l}\text { Prepare Process Memo for Mixing AP-102 (shown as } \\
5 \text { weekdays in P3) }\end{array}$ \\
\hline 3 & $15 \mathrm{~B} 42 \mathrm{D}$ & Blend \& Shim to AP-102 Tank Chemistry \\
\hline 5 & $15 \mathrm{~B} 32 \mathrm{~B}$ & Perform Batch Mixing Operation \\
\hline 5 & $15 \mathrm{~B} 34 \mathrm{~A}$ & LAW AP-102 Feed Qual Grab Sample \\
\hline 22 & $15 \mathrm{~B} 38 \mathrm{~A}$ & LAW AP-102 Anayze Feed Qual Sample \\
\hline 31 & $15 \mathrm{~B} 38 \mathrm{~B}$ & $\begin{array}{l}\text { LAW AP-102 Write/Issue Qual Sample Report (shown } \\
\text { as 22 weekdays in P3) }\end{array}$ \\
\hline 15 & $150 \mathrm{~B} 40$ & $\begin{array}{l}\text { Provide AN-105 Feed Batch Info from AP-102 to PC } \\
1 \text { (shown as 11 weekdays in P3) }\end{array}$ \\
\hline 106 & TOTAL & \\
\hline
\end{tabular}

Table A-17. Transfer Low-Activity Waste Feed to Vendor Tanks.

\begin{tabular}{|c|c|c|}
\hline Calendar days & $\begin{array}{c}\text { P3 schedule } \\
\text { activity ID }\end{array}$ & Activity title \\
\hline 9 & 15B44B & Deliver AN-105 Feed to AP-106 \\
\hline 9 & TOTAL & \\
\hline
\end{tabular}


Table A-18. RTP P3 Schedule.

\begin{tabular}{|c|c|c|c|c|c|}
\hline MLI & ACT & ITLE & OD & ES & F \\
\hline 30.8 & 3B25A & Write Process Test & & & \\
\hline $\begin{array}{l}130.825 \\
130 . \mathrm{B} 25\end{array}$ & $13 B 25 B$ & $\begin{array}{l}\text { Write process Test PLan tor Degas of } \\
\text { Degas AN-I05 using Process Test plan }\end{array}$ & 60 & 3APRO1 & lJUNO1 \\
\hline & & Add Diluent ( 25 kGal) Decant Pump Recirc AN-105 & 2 & $7 J U L 01$. & $8 \pi 0101$ \\
\hline 13 & 13В30В1 & Write Procedure (Xfer) for AN-105 to AP-102 & 20 & 5MARO1 & 3OMARO1 \\
\hline & 13В30В2 & Decant 250kgal Erom AN-I05 to AP-I02 & 6 & 9JUL01. & $14 \pi U L 01$. \\
\hline & $13 \mathrm{~B}$ & Write Procedure (Xfer) for AN-105 to AP-104 & 20 & 5MAR01 & 3OMARO 1 \\
\hline & $13 \mathrm{E}$ & Perform Xfer from AN-105 to AP-104 & 6 . & 15JUL01 & 20JUL01 \\
\hline & & Add Diluent water to $\mathrm{AN}-105$ & 5 & $21 \mathrm{JULO1}$ & 25JUL01 \\
\hline & & Prep Process Memo to Operate $M$ & 5 & 25APR01 & IMAYOI \\
\hline & & Operate Mixef pumps in AN-105 & 5 & $26 \pi \mathrm{J}$ & 30JUL01 \\
\hline & $13 \mathrm{~B}$ & Perform Grab Sampling Analysis of AN-105 & 3 & $31 J U I$ & 2AUGO1 \\
\hline & & settle solids in AN-105 & 30 & 3AUC & ISE \\
\hline & & Decant waste from AN-105 to AP-102 & 8 & $2 S E$ & $9 S 5$ \\
\hline & & Decant Waste from AN-105 to AP-104 & 8 & $10 s E$ & $17 \mathrm{~s}$ \\
\hline & & Write Xfer Procedure for AN-106 to AP-102 & 20 & $28 \pi \mathrm{U}$ & 24AC \\
\hline & $13 M$ & Transfer $800 \mathrm{kGal}$ from $\mathrm{AN}-106$ to $\mathrm{AP}-102$ & 10 & 25AUC & 3SE \\
\hline & & Write Xfer Procedure for AN-106 to AP-104 & 20 & $20 \mathrm{Cr}$ & 2700 \\
\hline & & Transfer $800 \mathrm{KGal}$ from AN-106 to AP-104 & 10 & $280 C^{\prime}$ & $6 \mathrm{NO}$ \\
\hline & 135 & Write Process Test Plan for Degas of SY-101 & 30 & $30 \mathrm{C}$ & $1 \pm N C$ \\
\hline & & Degas SY-101 using Process Test Plan & 60 & $17 \mathrm{DE}$ & $14 \mathrm{Er}$ \\
\hline & & Add Diluent .01m 32Kgal to sY-101 & 4 & $15 \mathrm{FE}$ & $18 \mathrm{E}$ \\
\hline & A1 & Prepare Process Memo & 5 & $4 J A$ & $10 \mathrm{~J}$ \\
\hline & & pare Transfer Procedure & 20 & $14 D E$ & $13 \mathrm{~J}$ \\
\hline & & Decant $538 \mathrm{kGal}$ from SY-101 to $S Y-102$ & 8 & $19 \mathrm{FE}$ & $26 F$ \\
\hline & & Add Diluent $500 \mathrm{KGal}$ of $.01 \mathrm{~m}$ Caustic to SY-101 & 8 & $27 \mathrm{FE}$ & $6 \mathrm{M}$ \\
\hline & & $\mathrm{Mix} S \mathrm{SY}-101$ & 5 & $7 \mathrm{MA}$ & $1 I M$ \\
\hline & & Perform Grab Sample \& Analyże SY-101 & 18 & $12 \mathrm{MA}$ & $29 \mathrm{M}$ \\
\hline & G & Settle sY-101 & 30 & $15 \mathrm{MD}$ & 13. \\
\hline & $H$ & Decant SY-101 to SY-102 $500 \mathrm{KGal}$ & 8 & $14 A E$ & 21 \\
\hline & & Write Xfer Erocedure for 102-SY to 102-AN & 20 & $27 \mathrm{FE}$ & 24 \\
\hline R3 35 & & Mix 102-sy with Process Memo from $13 \mathrm{P} 50 \mathrm{C}$ & 5 & $24 A F$ & 287 \\
\hline & & Transfer $800 \mathrm{kGal}$ from $102-\mathrm{SY}$ to $102-\mathrm{AN}$ & 15 & $29 \mathrm{AI}$ & 13 \\
\hline & & Decant $500 \mathrm{KGal}$ from $101-\mathrm{SY}$ to $102-\mathrm{SY}$ & 9 & $14 \mathrm{MA}$ & $22 \mathrm{M}$ \\
\hline & & Write xfer procedure for 102-AN to 102-AP & 20 & $26 \mathrm{M}$ & $20 \mathrm{P}$ \\
\hline & & Transfer $800 \mathrm{kGal}$ from $102-\mathrm{AN}$ to $102-\mathrm{AP}$ & 10 & 217 & 30 \\
\hline & & Write Xfer Procedure for $102-S Y$ to 107 -AN & 20 & $21 \mathrm{MP}$ & 17 \\
\hline & & Mix 102-sy with Process Memo from 13P50C & 5 & $23 \mathrm{MA}$ & 27 \\
\hline & & Transfer $800 \mathrm{KGal}$ from $102-\mathrm{SY}$ to $107-\mathrm{AN}$ & 15 & $28 \mathrm{MP}$ & 11 \\
\hline & & Write Xfer Procedure for 107-AN to 104-AP & 20 & 29 & $25 \mathrm{~J}$ \\
\hline & & Transfer $800 \mathrm{KGal}$ from $107-\mathrm{AN}$ to $104-\mathrm{AP}$ & 10 & $26 \mathrm{Jt}$ & \\
\hline 2 & & Prepare (Process Memo) Batch Mixing Plan & 5 & $2 \pi$ & 01 \\
\hline & & Perform Batch Mixing Operations & 5 & 100 & 01 \\
\hline 4 & 15 & LAW AP-102 Feed Qual Grab sample & 5 & 1500 & 01 \\
\hline & & LAW AP-102/4 Update Eeed Qual Grab Samp PM/TSAP & 10 & $2 J$ & 01 \\
\hline 150 & 15 & LAW AF-102 Updt Fd Ql Grab Samp Plan/Sch/obt Mtl & 22 & $28 \pi 0$ & $30 \pi$ \\
\hline 150 & 15 & LAW AP-102 Analyze Feed Qual sample & 22 & 200 & 01 \\
\hline & 15 & LAW AR-102 Write/Issue Qual Sample Report & 22 & $12 \mathrm{NO}$ & 01 \\
\hline 8 & 15 & LAW AP-102 Prep Feed Qual sample Lab Report & 15 & $11 \mathrm{NO}$ & $25 \mathrm{NC}$ \\
\hline & 15 & Draft Transmit Ltr Data to PC\#1 AN-105/AP-102 & 2 & $14 \mathrm{DEC} 01$ & $17 \mathrm{DE}$ \\
\hline & $15 \mathrm{E}$ & Obtain INHC Appr for Transmit Itr AN-105/AP-102 & 5 & $18 \mathrm{DE}$ & $27 \mathrm{DE}$ \\
\hline & 15 & FDH Transmit Data to DOE-RL AN-105/AP-102 & 2 & $28 \mathrm{DEC} 01$ & 31DEC01 \\
\hline & 15 & DOE-RI Iss Trnsmt Itr Data to PC\#I AN-105/AP-10 & 2 & 2JANO2. & $3 J A$ \\
\hline & & are Process Memo for Mixing AP-102 & 5 & AJUNO1 & $8 \pi$ \\
\hline & 15 & Orm AP-102 Mixing operation & 5 & 10SER01 & $14 \mathrm{SE}$ \\
\hline & $15 \mathrm{E}$ & lyze Process Control Samps in AP-102 & 13 & 15SEP01. & $27 \operatorname{SEP} 01$ \\
\hline & 158 & to AP-102 Tank Chemistry & 3 & SOCTO1 & $90 \mathrm{cT} 01$ \\
\hline & & ess Memo for Mixing $A P-102$ & 5 & 28SEP01 & $40 \mathrm{CTO} 01$ \\
\hline 150. & $15844 B$ & Transfer $700 \mathrm{Kgal}$ from $102 \mathrm{AP}$ to $106 \mathrm{AP}$ & 9 & 4JANO2 & 12JANO2 \\
\hline
\end{tabular}


HNF-2168

Revision 0

This page intentionally left blank. 
HNF-2168

Revision 0

\section{A.5 REFERENCES}

Grenard, C. E., and R. D. Claghorn, 1998, System Specification for the Double-Shell Tank System, HNF-SD-WM-TRD-007, Rev. 0, Numatec Hanford Corporation, Richland, Washington.

Kirkbride, R. A., G. K. Allen, P. J. Certa, A. F. Manuel, R. M. Orme, L. W. Shelton, E. J. Slaathaug, R. S. Wittman, and G. T. MacLean and D. L. Penwell (SESC), 1997, Tank Waste Remediation System Operation and Utilization Plan, HNF-SD-WM-SP-012, Rev. 0, Vol. I and II, Numatec Hanford Corporation, Richland, Washington.

Porter, P. E., 1996, Hanford Site Technical Database, WHC-SD-GN-CSWD-20012, Rev. 5, Richland, Washington.

Swita, W. R., 1998, Tank Waste Remediation System Retrieval and Disposal Mission Initial Updated Baseline Summary, HNF-1946, Rev. 1, Lockheed Martin Hanford Corporation, Richland, Washington.

Wittman, R., 1997, Verification and Validation of the Tank Waste Remediation System Hanford Tank Waste Operations Simulator, HNF-SD-TWR-RPT-006, Rev. 0, Numatec Hanford Corporation, Richland, Washington. 
HNF-2168

Revision 0

This page intentionally left blank. 


\begin{tabular}{|c|c|c|c|c|c|}
\hline \multirow{2}{*}{$\begin{array}{l}\text { To } \\
\text { Distribution } \\
\end{array}$} & \multirow{2}{*}{\multicolumn{3}{|c|}{$\begin{array}{l}\text { From } \\
\text { R. D. Claghorn, NHC }\end{array}$}} & \multicolumn{2}{|l|}{ Page 1 of 1} \\
\hline & & & & \multicolumn{2}{|l|}{ Date $3 / 4 / 98$} \\
\hline \multirow{2}{*}{\multicolumn{4}{|c|}{$\begin{array}{l}\text { Project Title/Work Order } \\
\text { Performance Requirements for the Double-She11 Tank System: } \\
\text { Phase 1, HNF-2168, Rev. } 0\end{array}$}} & \multicolumn{2}{|c|}{ EDT No. 622702} \\
\hline & & & & \multicolumn{2}{|c|}{ ECN No. } \\
\hline Name & MSIN & $\begin{array}{c}\text { Text } \\
\text { With All } \\
\text { Attach. }\end{array}$ & Text Only & $\begin{array}{l}\text { Attach./ } \\
\text { Appendix } \\
\text { Only } \\
\end{array}$ & $\begin{array}{c}\text { EDT/ECN } \\
\text { Only }\end{array}$ \\
\hline $\begin{array}{l}\text { Central Files } \\
\text { DOE Reading Room }\end{array}$ & $\begin{array}{l}\text { B1-07 } \\
\text { A1-65 }\end{array}$ & $\begin{array}{l}x \\
x\end{array}$ & & & \\
\hline $\begin{array}{l}\text { S. K. Baker } \\
\text { P. J. Certa } \\
\text { A. F. Choho } \\
\text { R. D. Claghorn (3) } \\
\text { T. J. Conrads } \\
\text { M. A. Delamare } \\
\text { J. S. Garfield } \\
\text { C. E. Grenard } \\
\text { J. G. Kristofzski } \\
\text { R. P. Marshall } \\
\text { S. M. 0'Toole } \\
\text { I. G. Papp } \\
\text { B. B. Peters } \\
\text { R. W. Powe17 } \\
\text { W. T. Thompson } \\
\text { R. L. Treat }\end{array}$ & $\begin{array}{l}H 5-49 \\
H 5-61 \\
H 6-35 \\
H 5-49 \\
H 5-25 \\
H 5-61 \\
H 5-49 \\
H 5-61 \\
\text { R2-12 } \\
H 5-61 \\
G 3-21 \\
H 5-49 \\
H 5-03 \\
H 5-03 \\
G 3-21 \\
H 5-03\end{array}$ & $\begin{array}{l}x \\
x \\
x \\
x \\
x \\
x \\
x \\
x \\
x \\
x \\
x \\
x \\
x \\
x \\
x \\
x\end{array}$ & & . & \\
\hline TWR Proj File CPF \#28 & H6-08 & $x$ & & & \\
\hline
\end{tabular}

